\title{
A Generally Weighted Moving Average Chart for Time Between Events
}

N. Chakraborty ${ }^{1, \mathrm{a}}$, S.W. Human ${ }^{1, \mathrm{~b}}$, N. Balakrishnan ${ }^{2, \mathrm{c}}$

${ }^{1}$ Department of Statistics, University of Pretoria, Pretoria, 0002, Lynnwood Road, Hillcrest, South Africa

${ }^{2}$ Department of Mathematics and Statistics, McMaster University, Hamilton, Ontario, Canada L8S 4K1

${ }^{\mathrm{a} C}$ Corresponding author: e-mail niladriorama@gmail.com

${ }^{\mathrm{b}}$ Corresponding author: e-mail: schalk.human@up.ac.za

${ }^{c}$ Corresponding author: e-mail bala@mcmaster.ca

\begin{abstract}
Shewhart-type attribute charts are known to be inefficient for small changes in monitoring nonconformities. An alternative way is to use a time-weighted chart to monitor the time between events (TBE). We propose a one-sided Generally Weighted Moving Average control chart to monitor the time between events (TBE); regarded as the GWMA-TBE chart. To aid the implementation of the chart, the necessary design parameters are provided. An extensive performance analysis shows that the GWMA-TBE chart is better than the well-known EWMA and Shewhart charts at detecting very small to moderate changes. Finally, a summary and some conclusions are provided.
\end{abstract}

\section{Keywords}

GWMA chart; Time between events; Average Run-length; Simulation; Markov chain 


\section{Introduction}

Since its introduction by Walter A. Shewhart in the 1920's, statistical quality control (SQC) has found a variety of applications ranging from health care monitoring to financial fraud detection. In general, an item that does not comply with the standards is labelled a nonconforming or defective item. The traditional Shewhart-type attribute charts such as the $c$-chart or $p$-chart (see Montgomery, 2009) are used to monitor a process when the number of nonconformities or the number of defective items is of interest; these charts use the number of nonconformities or the number of defective items in regular, typically equi-spaced, time intervals and are known to be ineffective in detecting small shifts. Such rare events are frequently found in today's highperforming technological environment wherein the rate of failures can be very small. An alternative and perhaps more appealing way to monitor the rate of failures (called the Poisson rate) is to use control charts based on the inter-arrival times of nonconforming items. These charts are especially useful when the occurrence of defective items is rare and the rate of occurrence is very small. Such charts are called time between events (TBE) charts and are typically based on the following two assumptions: (a) the occurrence of failures in a process follows a homogeneous Poisson process, and (b) the inter-arrival times between two consecutive events follow an exponential distribution. A number of TBE control charts can be found in the literature. The Cumulative Sum (CUSUM) chart to monitor the time between events, which is simply called the exponential CUSUM chart, was proposed by Vardeman and Ray (1985); the optimal design of the exponential CUSUM chart was given by Gan (1994) and an algorithm for computing the average run-length ( $A R L$ ) of the chart was subsequently given by Gan and Choi 
(1994). The design and performance of the Exponentially Weighted Moving Average (EWMA) chart for the exponential distribution was studied by Gan (1998). A Shewhart-type chart for the time between events (also assuming an exponential distribution) was studied by Xie et al. (2002). In addition, Zhang et al. (2007) studied the performance of the Shewhart-type time between events chart for the gamma distribution.

Time-weighted control charts such as the CUSUM and EWMA have been shown to be efficient in detecting small sustained upward or downward step shifts in a process - see Montgomery (2009) for more details and references on the theory, design and application of the classical EWMA and CUSUM charts for the normal distribution. Capitalising on the efficiency of the EWMA chart, Sheu and Lin (2003) proposed a Generally Weighted Moving Average (GWMA) control chart for the normal distribution; this chart is a generalisation of the EWMA chart and has been shown to be more effective than the EWMA, CUSUM and Shewhart-type charts (see Hsu et al., 2009) in detecting very small shifts. For more details on the recent developments on the GWMA charts, the interested reader is referred to the works of Sheu and Yang (2006), Sheu and Chiu (2007), Chiu and Sheu (2008), Chiu (2009), Sheu and Hsieh (2009), Sheu et al. (2012), Teh et al. (2012), Sheu et al. (2013), Huang et al. (2014), Huang (2014), Lu (2015), Aslam et al. (2015) and Chakraborty et al. (2016).

As noted, for example, by Zhang et al. (2005) and Balakrishnan et al. (2015), the quick detection of any deterioration in a process is of utmost importance from a practical point of view. In the current context, i.e., monitoring the time between events, process deterioration can occur following an increase in the failure rate. So, for a high quality process, a small reduction in 
process performance needs to be detected as early as possible. This does not imply that process improvement is not important. Quite to the contrary, as mentioned by Maravelakis and Castagliola (2009), an improvement in a process usually occurs after corrective action has taken place, but the time of the change is usually known and a control chart is not needed to detect the change/improvement. For this reason, a one-sided control chart to detect process deterioration is pursued here.

In this article, we construct a one-sided GWMA chart based on the gamma distribution for monitoring the time between events (often regarded as the time between failures); this chart is labelled the GWMA-TBE chart and can be used in detecting a sustained downward step shift in a process. The proposed chart includes the one-sided EWMA and Shewhart charts as special cases, and it differs in two ways from the currently available TBE charts: (i) The interdependency of the GWMA charting statistics is explicitly taken into consideration, and (ii) we specifically focus on a one-sided GWMA-TBE chart based on the gamma distribution. The proposed one-sided GWMA-TBE chart is thus run-length unbiased (by construction) which is unlike the currently available two-sided control charts (based on the exponential distribution) which are biased. Note that a control chart is "biased" when the out-of-control average run-length (denoted by $\left.A R L_{1}\right)$ is larger than its in-control value (denoted by $A R L_{0}$ ) and therefore takes longer to detect a shift in the process; this is an undesirable property and normalizing transformations often do not completely eliminate this problem.

The rest of this article is organised as follows: The general theory and background on the GWMA-TBE chart are given in Section 2. In Section 3, the run-length distribution for the 
scenario when the parameters are known is studied; this includes the in-control (IC) design and the out-of-control (OOC) performance. The GWMA-TBE chart for the scenario when the parameters are unknown is studied in Section 4; this includes the effects of parameter estimation on the performance as well as the design of the Phase II GWMA-TBE chart. In Section 5, we show how the proposed chart can also be used to monitor the variance of a normal distribution. An illustrative example is provided in Section 6 and we finally conclude with some remarks in Section 7.

\section{The GWMA Time Between Events (GWMA-TBE) control chart}

Let the random variables $Y_{j} \sim$ iid $\operatorname{Exp}(\theta), j=1,2,3, \ldots$, denote the inter-arrival times between consecutive failures in a homogeneous Poisson process with rate parameter $1 / \theta$. The continuous random variable $X=\sum_{j=1}^{k} Y_{j}$, which is the sum of the inter-arrival times of $k$ consecutive failures, then denotes the time until the $k^{\text {th }}$ failure and is known to follow a $\operatorname{Gamma}(k, \theta)$ distribution. The probability density function (p.d.f.) of $X$ is given by

$$
f(x ; k, \theta)=\frac{e^{-x / \theta} x^{k-1}}{\Gamma(k) \theta^{k}}, x>0, \theta>0 \text { and } k>0 .
$$

Note the following regarding the p.d.f. in (1):

i. The expected value and variance of $X$ are $E(X)=k \theta$ and $\operatorname{var}(X)=k \theta^{2}$;

ii. Although the parameter $k>0$ can theoretically be any positive real number, in the developments that follow it is assumed that $k$ is a known/specified positive integer, i.e., 
$k=1,2,3, \ldots$, and selected by the practitioner. For example, if we set $k=3$, the random variable $X$ denotes the total time between three consecutive failures;

iii. If $k=1$, the p.d.f. in (1) reduces to that of an exponential distribution and we would be interested in monitoring the time until one failure;

iv. It is assumed that $\theta_{0}$ denotes the in-control (IC) standard/value for the parameter $\theta$; this is known as the "standard known" case and denoted by Case K. We primarily focus on the scenario when the parameter $\theta$ is known, but we also deal with the situation when $\theta$ is unknown and estimated from an in-control (IC) Phase I reference sample before online monitoring can start in Phase II; the latter scenario is referred to as the "standard unknown" case and denoted by Case U;

v. Our objective is to construct a control chart to monitor $\theta$ for a sustained downward step shift, i.e., a decrease in the inter-arrival times, which would be indicative of deterioration in the process. The key focus is to detect small or very small shifts as quickly as possible.

The GWMA-TBE control chart is constructed by taking a weighted average of a sequence of the $X_{i}{ }^{\prime} s$. To this end, let $N$ be the discrete random variable denoting the number of samples until the next occurrence of an event since its last occurrence. Then, by summing over all values of $N$ , we can write

$$
\sum_{i=1}^{\infty} \operatorname{Pr}[N=i]=\sum_{i=1}^{t} \operatorname{Pr}[N=i]+\operatorname{Pr}[N>t]=1
$$

A generally weighted moving average (GWMA) is a weighted moving average (WMA) of a sequence of $X_{i}$ statistics where the probability $\operatorname{Pr}[N=i]$ is regarded as the weight $w_{i}$ for the $i^{\text {th }}$ 
most recent statistic $X_{t-i+1}$ among the last $t$ of $X_{i}$ statistics. In other words, the probability $\operatorname{Pr}[N=1]$ is the weight $w_{1}$ for the latest observation $X_{t}$ and the probability $\operatorname{Pr}[N=t]$ is the weight $w_{t}$ for the most out-dated observation $X_{1}$. The probability $\operatorname{Pr}[N>t]$ is considered as the weight for the starting value, denoted by $Z_{0}$, which is typically taken as the in-control (IC) expected value of the statistic under consideration, i.e., $Z_{0}=E\left(X_{i} \mid \mathrm{IC}\right)=k \theta_{0}$. Therefore, the charting statistic for the GWMA-TBE chart is defined as

$$
Z_{t}=\sum_{i=1}^{t} \operatorname{Pr}[N=i] X_{t-i+1}+\operatorname{Pr}[N>t] Z_{0} \text { for } t=1,2,3, \ldots
$$

As in Sheu and Lin (2003), the distribution of $N$ is taken to be $\operatorname{Pr}[N=i]=q^{(i-1)^{\alpha}}-q^{i^{\alpha}}$, where $0 \leq q<1$ and $\alpha>0$ are the two parameters; this is the discrete two-parameter Weibull distribution (see Nakagawa and Osaki, 1975). So, the weights are given by $w_{i}=q^{(i-1)^{\alpha}}-q^{i^{\alpha}}$. By substituting $Z_{0}=k \theta_{0}$ and the probability mass function (p.m.f.) of the two-parameter discrete Weibull distribution in equation (3), the charting statistic for the GWMA-TBE chart simplifies to

$$
Z_{t}=\sum_{i=1}^{t}\left(q^{(i-1)^{\alpha}}-q^{i^{\alpha}}\right) X_{t-i+1}+q^{t^{\alpha}} k 0 \theta \text { for } t=1,2,3, \ldots
$$

Note that the GWMA-TBE chart reduces to an EWMA chart when $\alpha=1$ and $q=1-\lambda$, where $0<\lambda \leq 1$ is the smoothing parameter of the EWMA chart. The EWMA chart further reduces to the Shewhart chart when $q=0$ and $\alpha=1$. The GWMA chart can therefore be viewed as a generalization of the EWMA and Shewhart-type charts with an additional parameter $\alpha$ which provides more flexibility in designing the chart. We introduce the EWMA-TBE and the Shewhart-TBE charts as special cases. 
A closer look at the choice of weights for the GWMA chart shows that the weights $w_{i}=q^{(i-1)^{\alpha}}-q^{i^{\alpha}}, i=1,2,3, \ldots$, are decreasing in $i$ for a fixed $0<q<1$ and $0<\alpha \leq 1$; this implies that more weight is given to more recent observations in the sequence of $X_{i}{ }^{\prime} s$. A proof of this result is given in the Appendix.

The in-control (IC) expected value and variance of the charting statistic $Z_{t}$ are given by

$$
E\left(Z_{t} \mid \mathrm{IC}\right)=\sum_{i=1}^{t}\left(q^{(i-1)^{\alpha}}-q^{i^{\alpha}}\right) E\left(X_{t-i+1}\right)+q^{t^{\alpha}} k \theta_{0}=k \theta_{0}
$$

and

$$
\operatorname{var}\left(Z_{t} \mid \mathrm{IC}\right)=\sum_{i=1}^{t}\left(q^{(i-1)^{\alpha}}-q^{i^{\alpha}}\right)^{2} \operatorname{var}\left(X_{t-i+1}\right)=Q_{t} k \theta_{0}^{2}
$$

respectively, where $Q_{t}=\sum_{i=1}^{t}\left(q^{(i-1)^{\alpha}}-q^{i^{\alpha}}\right)^{2}$.

The exact time-varying (symmetric) control limits $\left(U C L_{e} \& L C L_{e}\right)$ and centerline $\left(C L_{e}\right)$ of a two-sided GWMA-TBE chart are given by

$$
U C L_{e} / L C L_{e}=k \theta_{0} \pm L \sqrt{Q_{t} k \theta_{0}^{2}} \text { and } C L_{e}=k \theta_{0}
$$

where $L>0$ is the distance of the control limits from the centerline and the subscript " $e$ " denotes the exact control limits.

The steady-state control limits are used when the process has been running for several time periods and are based on the asymptotic variance of the charting statistic (see Lucas and Saccucci, 1990). The steady-state control limits and the centerline are given by

$$
U C L_{s} / L C L_{s}=k \theta_{0} \pm L \sqrt{Q k \theta_{0}^{2}} \text { and } C L_{s}=k \theta_{0} \text {, }
$$


where the subscript " $s$ " denotes the steady-state control limits and $Q=\lim _{t \rightarrow \infty} Q_{t}$.

As we are primarily interested in detecting deterioration in the process, i.e., a decrease in the waiting times between failures, only a lower control limit is used in the design and implementation of the proposed GWMA-TBE chart; this limit is given by

$$
L C L_{s}=k \theta_{0}-L \sqrt{Q k \theta_{0}^{2}}
$$

A GWMA-TBE chart for detecting improvement in a process can be designed in a similar manner but not pursued here any further for the sake of brevity.

The following two points are worth noting here:

i. The quantity $Q_{t}$ is a monotonically increasing and convergent function of $t$ (see the Appendix for more details). To this end, Table 1 shows the value of $Q_{t}$ for different choices of the parameters $(q, \alpha)$ and various values of $t$. We observe that the change in $Q_{t}$ is almost negligible for $t \geq 10$. Due to the quick convergence of $Q_{t}$, the exact control limits also quickly converge towards the steady-state limits. Hence, the steady-state lower control limit is used in order to simplify the application/implementation of the proposed chart. For the sake of notational simplicity, we will use $L C L$ hereafter to denote the steady-state lower control limit in (9);

ii. If any $Z_{t}$ plots on or below $L C L$, the process is declared out-of-control (OOC) and a search for assignable causes is initiated. Otherwise, the process is considered to be in-control (IC), which implies no change in $\theta$ has occurred, and the charting procedure continues on.

In the ensuing section, we discuss the design of the proposed GWMA-TBE chart in more detail. 
Table 1: $Q_{t}$ values for different $(q, \alpha)$ combinations.

\begin{tabular}{|c|c|c|c|}
\hline $\boldsymbol{t}$ & $\boldsymbol{q}=\mathbf{0 . 5}, \boldsymbol{\alpha}=\mathbf{0 . 5}$ & $\boldsymbol{q}=\mathbf{0 . 5}, \boldsymbol{\alpha}=\mathbf{0 . 9}$ & $\boldsymbol{q}=\mathbf{0 . 5}, \boldsymbol{\alpha}=\mathbf{1 . 3}$ \\
\hline $\mathbf{5}$ & 0.2751 & 0.3208 & 0.3691 \\
\hline $\mathbf{1 0}$ & 0.2773 & 0.3215 & 0.3691 \\
\hline $\mathbf{5 0}$ & 0.2778 & 0.3215 & 0.3691 \\
\hline $\mathbf{1 0 0}$ & 0.2778 & 0.3215 & 0.3691 \\
\hline $\boldsymbol{t}$ & $\boldsymbol{q}=\mathbf{0 . 7}, \boldsymbol{\alpha}=\mathbf{0 . 5}$ & $\boldsymbol{q}=\mathbf{0 . 7}, \boldsymbol{\alpha}=\mathbf{0 . 9}$ & $\boldsymbol{q}=\mathbf{0 . 7}, \boldsymbol{\alpha}=\mathbf{1 . 3}$ \\
\hline $\mathbf{5}$ & 0.1074 & 0.1555 & 0.2227 \\
\hline $\mathbf{1 0}$ & 0.1107 & 0.1614 & 0.2239 \\
\hline $\mathbf{5 0}$ & 0.1129 & 0.1618 & 0.2239 \\
\hline $\mathbf{1 0 0}$ & 0.1129 & 0.1618 & 0.2239 \\
\hline $\boldsymbol{t}$ & $\boldsymbol{q}=\mathbf{0 . 9} \boldsymbol{\alpha}=\mathbf{0 . 5}$ & $\boldsymbol{q}=\mathbf{0 . 9} \boldsymbol{\alpha}=\mathbf{0 . 9}$ & $\boldsymbol{q}=\mathbf{0 . 9} \boldsymbol{\alpha}=\mathbf{1 . 3}$ \\
\hline $\mathbf{5}$ & 0.0132 & 0.0274 & 0.0667 \\
\hline $\mathbf{1 0}$ & 0.0143 & 0.0360 & 0.0866 \\
\hline $\mathbf{5 0}$ & 0.0160 & 0.0427 & 0.0887 \\
\hline $\mathbf{1 0 0}$ & 0.0163 & 0.0427 & 0.0887 \\
\hline
\end{tabular}




\section{The design and implementation of GWMA-TBE chart}

Performance measures are needed to design and compare the performance of control charts. The traditional approach of evaluating a control chart is to obtain the run-length distribution and its associated characteristics. The run-length is a discrete random variable that represents the number of samples which must be collected (or, equivalently, the number of charting statistics that must be plotted) in order for the chart to detect a shift or give a signal. An intuitively appealing and popular measure of a chart's performance is the average run-length ( $A R L)$, which is the expected number of charting statistics that must be plotted in order for the chart to signal (see Human and Graham, 2007). Clearly, for an efficient control chart, one would like to have the in-control $A R L$ (denoted $A R L_{0}$ ) to be "large" and the out-of-control $A R L$ (denoted $A R L_{1}$ ) to be "small". Although other measures such as the standard deviation of the run-length (SDRL) and various upper and lower percentiles could be and have been used to supplement the evaluation of control charts, the $A R L$ is the most widely used measure due to its intuitive appealing interpretation. Therefore, we use primarily the $A R L$ to design and compare the performance of the proposed GWMA-TBE chart.

The design of a control chart typically involves solving for the combination of the chart's parameters, i.e., $q, \alpha$ and $L$, so as to obtain a pre-specified in-control average run-length denoted by $A R L_{0}^{*}$ for a given or selected value of $k$. The computational aspects of the run-length distribution for the GWMA-TBE chart are discussed next; this is followed by the design of the GWMA-TBE chart. 


\subsection{The run-length distribution of the GWMA-TBE chart}

There are numerous methods to calculate the run-length distribution of a time-weighted chart like the GWMA chart and we investigate here three approaches: (i) An exact approach based on closed-form expressions, (ii) A Markov chain approach, and (iii) Monte Carlo Simulation. Each method is discussed in more detail below along with their pros and cons. It should be noted that ultimately we used computer simulation and double-checked the results by using the Markov chain approach for the EWMA-TBE chart; these results are available upon request.

\subsubsection{Exact approach}

Suppose the run-length random variable is denoted by $R$ and that $A_{i}$ denotes the signalling event at the $i^{\text {th }}$ sample. The non-signalling event is therefore given by $A_{i}^{c}=\left[Z_{i}>L C L\right]$ for $i=$ $1,2,3, \ldots$ Then, in general, the run-length distribution can be written as

$\operatorname{Pr}[R=r]=\operatorname{Pr}\left[\left\{\bigcap_{i=1}^{r-1} A_{i}^{\mathrm{c}}\right\} \bigcap_{r}\right]$, for $r=1,2,3, \ldots$. For any $i \geq 1$, we can re-write the event $A_{i}^{c}=\left[Z_{i}>L C L\right]$, as $A_{i}^{c}=\left[X_{i}>L_{i}\right]$, where $L_{1}=\frac{L C L-q k \theta_{0}}{1-q}$ and

$$
L_{i}=\frac{L C L-\sum_{j=2}^{i}\left(q^{(j-1)^{\alpha}}-q^{j^{\alpha}}\right) X_{i-j+1}-q^{i^{\alpha}} k \theta_{0}}{1-q}, i=2,3,4, \ldots
$$

The run-length distribution can therefore be written as

$\operatorname{Pr}[R=1]=1-\operatorname{Pr}\left[X_{1}>L_{1}\right]$ and $\operatorname{Pr}[R=r]=I_{r-1}-I_{r}$,

where $I_{\mathrm{r}}=\operatorname{Pr}\left[\bigcap_{i=1}^{r} A_{i}^{c}\right]=\operatorname{Pr}\left[\bigcap_{i=1}^{r}\left\{X_{i}>L_{i}\right\}\right]$ for $r=2,3,4, \ldots$; see the Appendix for more details. 
Since the $X_{i}$ 's are assumed to be independent $\operatorname{Gamma}(k, \theta)$ random variables, we have

$$
I_{r}=\int_{L_{1}}^{\infty} \int_{L_{2}}^{\infty} \ldots \int_{L_{r}}^{\infty}\left\{\prod_{i=1}^{r} f\left(x_{i} ; k, \theta\right) d x_{i}\right\}
$$

where $f\left(x_{i} ; k, \theta\right)$ is the p.d.f. given in (1).

The $A R L$ can also be expressed in terms of $I_{\mathrm{r}}$ as (see the Appendix for more details)

$$
A R L=1+\sum_{r=1}^{\infty} I_{\mathrm{r}}
$$

To analytically evaluate expressions (12) and (13) is time-consuming and uneconomical for two reasons:

a. The lower bounds in the integrals of $I_{r}$, i.e., the $L_{i}$ 's given in equation (12), are mutually dependent and functions of the sequence of preceding statistics $X_{1}, X_{2}, \ldots, X_{i-1}$; these bounds cannot be economically recursively updated and is therefore a computationally expensive approach;

b. The number of terms in expression (12) that needs to be evaluated increases dramatically as $r$ increases;

As mentioned before, if we set $q=0$ and $\alpha=1$, we obtain the Shewhart-TBE chart. In this special case, the lower control limit in (10) reduces to $L_{i}=L C L$ for $i \geq 1$ and the probability of a signal is given by $\operatorname{Pr}\left[X_{i} \leq L C L \mid X_{i} \sim \operatorname{Gamma}(k, 1 / \delta)\right]$, where the lower control limit can be obtained by solving the expression $\left.\operatorname{Pr}\left[X_{i} \leq L C L \mid X_{i} \sim \operatorname{Gamma}(k, 1)\right]\right]=1 / A R L_{0}^{*}$. 


\subsubsection{Markov chain approach}

For the EWMA charts, using the Markov chain approach, the probability mass function (p.m.f.), $A R L$ and the variance (VARL) of the run-length random variable $R$ can be obtained as (see

Theorems 5.2 and 7.4 of $\mathrm{Fu}$ and Lou, 2003), $P[R=r]=\xi \mathbf{Q}^{r-1}(\mathbf{I}-\mathbf{Q}) 1$ for $r=1,2,3, \ldots$, $A R L=\xi(\mathbf{I}-\mathbf{Q})^{-1} 1$ and $V A R L=\xi(\mathbf{I}+\mathbf{Q})(\mathbf{I}-\mathbf{Q})^{-2} 1-A R L^{2}$, where the sub-matrix matrix $\mathbf{Q}=\mathbf{Q}_{v \times \nu}$ is called the essential transition probability sub-matrix, $\mathbf{I}_{v \times \nu}$ is the identity matrix, $\boldsymbol{\xi}_{1 \times \nu}$ is the 'initial probability vector' such that $\xi_{j}=1$ when the process mean i.e. $k \theta_{0}$, is in the $j^{\text {th }}$ state and $\xi_{j}=0$ for all other $j$ with $\sum_{j=1}^{v} \xi_{j}=1,1_{v \times 1}=(1,1, \ldots, 1)^{\mathrm{T}}$ is the unit vector and $v$ denotes the number of transient states in the state space $\Omega$. The Markov chain results for the one-sided EWMA-TBE chart are available upon request from the corresponding author. The interested reader is also referred to the work by Graham et al. (2011a and 2011b) for more details.

The prime obstacle using the Markov chain approach for the GWMA chart is the fact that the GWMA chart's plotting statistic cannot be viewed as following a first-order Markov chain. In fact, obtaining $Z_{t}$ requires all the $X_{t}$ 's from start-up, i.e., $X_{t}, X_{t-1}, \ldots, X_{0}$. This complication arises due to the repeated exponentiation (so-called "bottom-up" tetration) in the weights $w_{i}=q^{(i-1)^{\alpha}}-q^{i^{\alpha}}$ of the charting statistics. This implies that $Z_{t}$ depends on all $Z_{t-1}, Z_{t-2}, \ldots, Z_{1}$. To this end, note that the probability $\operatorname{Pr}\left[Z_{t} \mid Z_{t-1}, \ldots, Z_{1}\right]$ can be approximated by $\operatorname{Pr}\left[Y_{t}^{*} \mid Y_{t-1}^{*}\right]$, where $Y_{t}^{*}=\left(Z_{t}, \ldots, Z_{t-m+1}\right)$ and $m$ is the threshold beyond which the weights are approximately zero (see Appendix). So, $\left\{Y_{t}^{*}, t=m, m+1, m+2, \ldots\right\}$ becomes a first-order Markov chain and we can use the results from Fu and Lou (2003) for $t=m, m+1, \ldots$. For $t=1,2, \ldots, m-1$, one still has 
to use the exact approach. The major difficulty in using the Markov Chain approach for the GWMA-TBE chart is in defining the state-space of the first-order Markov chain \{ $\left.Y_{t}^{*}, t=m, m+1, m+2 \ldots\right\}$ as it depends on the state-space of $\left(Z_{t}, \ldots, Z_{t-m+1}\right)$, which is the Cartesian product $\Omega^{m}, t=m, m+1, m+2 \ldots \ldots$

Due to the above-mentioned difficulties with the exact approach and the Markov chain approach, extensive simulation has been used to calculate the run-length distribution for the proposed GWMA-TBE chart. To this end, it is important to note that, Sheu and Lin (2003), Sheu and Yang (2006) and $\mathrm{Lu}(2015)$ also mentioned that it is difficult to obtain the run-length distribution of the GWMA charts by using the exact approach or the Markov chain approach. The simulation approach is discussed next.

\subsubsection{Monte Carlo simulation approach}

The simulation algorithm uses the stochastic representation of the GWMA-TBE chart and is done according to the following five steps:

i. Select a combination of the design parameters, i.e., $(q, \alpha, L)$, set $\theta_{0}=1$ and calculate the control limit according to expression (9);

ii. Generate an individual observation from a $\operatorname{Gamma}(k, 1)$ distribution and calculate the charting statistic $Z_{t}$ according to expression (4) with the starting value taken as $Z_{0}=k$;

iii. If $Z_{t}>L C L$, the process is considered to be in-control and a run-length counter is incremented;

iv. Steps (ii) and (iii) are repeated and $Z_{t}$ is sequentially updated until $Z_{t} \leq L C L$; when this event occurs, a signal is given and the process is declared to be out-of-control. The simulation stops and the run-length is recorded; 
v. Steps (ii)-(iv) are repeated 10,000 times.

We have also used simulation for the EWMA-TBE chart in order to be consistent and be able to compare them with the Markov chain approach. The 10,000 simulated run-lengths can be used to empirically calculate the characteristics of the run-length distribution.

\subsection{The in-control (IC) design of the GWMA-TBE chart}

For a given or chosen value of $k$, the two parameters $q$ and $\alpha$ are varied over a certain range and for each $(q, \alpha)$ combination, the values of the charting constant, i.e. $L>0$, are obtained so that the attained in-control $A R L$ is close to (in this case slightly above or below due to the use of simulation) the nominal or specified value $A R L_{0}^{*}$. The typical industry standards for the $A R L_{0}^{*}$ are 370 or 500 and we consider the former in our study. The typical recommendation for the smoothing parameter $0<\lambda \leq 1$ for an EWMA chart is to choose smaller values for smaller shifts (see Montgomery, 2009, page 423). Because the GWMA chart reduces to an EWMA chart when $q=1-\lambda$ and $\alpha=1$, a larger value of $q$, i.e. closer to 1 , should be a reasonable choice for the GWMA chart to detect small shifts. To this end, Sheu and Lin (2003) noted that $(q, \alpha)$ combinations in the intervals $0.5 \leq q \leq 0.9$ and $0.5 \leq \alpha<1$ enhanced the sensitivity of the GWMA- $\bar{X}$ chart and outperformed the EWMA- $\bar{X}$ chart for small shifts (i.e., less than 1 standard deviations in the location). The same range of $(q, \alpha)$ values were also considered by Sheu and Yang (2006), Teh et al. (2012), Sheu et al. (2013), and Lu (2015). In our simulation

study, we set $k=1,2,3,4,5$ and considered the range $q=0.5,0.6,0.7,0.8,0.9,0.95$ and $\alpha=$ $0.5,0.6,0.7,0.8,0.9,1.0,1.3$, respectively. 
Using simulation along with a grid search algorithm, we obtained the charting constant $L>0$ for the chosen $(q, \alpha)$ combination and specified value of $k$, so that the attained $A R L_{0}$ is approximately equal to $A R L_{0}^{*}=370$; the values of $L$ determined in this way are presented in Table 2 along with the attained $A R L_{0}$ values. The $L$ values in Table 2 will be useful for the design and implementation of the GWMA-TBE chart; this includes designing an EWMA or Shewhart-type TBE chart. For example, if we choose $k=2$ and let $q=0.8, \alpha=0.7$, a value of $L=1.953$ leads to an attained $A R L_{0}$ equal to 370.05 . To highlight the design parameters of the EWMA and Shewhart-type TBE charts, the row for $\alpha=1.0$ have been highlighted.

From Table 2, we note in general that multiple combinations of the parameters $(q, \alpha, L)$ will yield the same $A R L_{0}$ for some chosen or specified value of $k$. This is somewhat challenging because, apart from desiring a sufficiently large $A R L_{0}$, the $A R L_{1}$ should be small for an effective GWMA-TBE chart. Therefore, the $(q, \alpha, L)$ combination with the minimum $A R L_{1}$ for a specified shift $\delta$ is said to be the optimal combination. The optimal design of the GWMA-TBE chart consists of specifying the desired $A R L_{0}$ and $A R L_{1}$ values as well as the magnitude of the process shift that is anticipated and then select the $(q, \alpha, L)$ combination that provides the desired $A R L$ performance; typically, the $(q, \alpha, L)$ combination with the minimum $A R L_{1}$ is selected. The solution to this problem is an optimization problem in 3-dimensional space. Although a detailed study on the optimal design for the GWMA-TBE chart is out-of-scope for this paper, in the next section we investigate and comment on the "near optimal" design given the out-of-control (OOC) $A R L$ values for different shift sizes. 
Table 2: Values of $L$ for the GWMA-TBE chart for different $(q, \alpha)$ when $k=1,2,3,4,5$ and $A R L_{0}^{*}=370$.

\begin{tabular}{|c|c|c|c|c|c|c|c|c|c|c|c|c|c|c|c|}
\hline \multirow{2}{*}{\begin{tabular}{|l|} 
\\
$k$ \\
\end{tabular}} & \multirow{3}{*}{$\begin{array}{c}\alpha \\
0.50\end{array}$} & \multicolumn{12}{|c|}{$q$} & \multirow{2}{*}{\multicolumn{2}{|c|}{$\begin{array}{c}\begin{array}{c}\text { Shewhar } \\
\text { t }\end{array} \\
q=0\end{array}$}} \\
\hline & & \multicolumn{2}{|c|}{0.5} & \multicolumn{2}{|c|}{0.6} & \multicolumn{2}{|c|}{0.7} & \multicolumn{2}{|c|}{0.8} & \multicolumn{2}{|c|}{0.9} & \multicolumn{2}{|c|}{0.95} & & \\
\hline 1 & & 1.3 & 36 & 1.4 & 37 & 1.5 & 37 & 1.5 & 37 & 1.6 & 37 & 1.5 & 37 & & \\
\hline & & 43 & $\begin{array}{c}9.6 \\
4\end{array}$ & 27 & $\begin{array}{c}0.5 \\
0\end{array}$ & 12 & $\begin{array}{c}0.2 \\
0\end{array}$ & 94 & $\begin{array}{c}1.1 \\
9\end{array}$ & 20 & $\begin{array}{c}1.3 \\
4\end{array}$ & 52 & $\begin{array}{c}0.3 \\
4\end{array}$ & & \\
\hline & 0.60 & 1.3 & 37 & 1.4 & 37 & 1.5 & 36 & 1.6 & 37 & 1.7 & 37 & 1.6 & 37 & & \\
\hline & & 84 & 2.6 & 82 & 2.2 & 85 & 9.9 & 87 & 1.2 & 28 & 0.9 & 15 & 0.2 & & \\
\hline & & & 4 & & 1 & & 4 & & 7 & & 6 & & 7 & & \\
\hline & 0.70 & 1.4 & 37 & 1.5 & 37 & 1.6 & 37 & 1.7 & 37 & 1.8 & 37 & 1.6 & 37 & & \\
\hline & & 13 & $\begin{array}{c}2.2 \\
2\end{array}$ & 20 & $\begin{array}{c}0.8 \\
2\end{array}$ & 35 & $\begin{array}{c}0.0 \\
2\end{array}$ & 50 & $\begin{array}{c}0.0 \\
6\end{array}$ & 06 & $\begin{array}{c}0.9 \\
6\end{array}$ & 89 & $\begin{array}{c}0.5 \\
5\end{array}$ & & \\
\hline & 0.80 & 1.4 & 36 & 1.5 & 37 & 1.6 & 37 & 1.7 & 37 & 1.8 & 37 & 1.7 & 37 & & \\
\hline & & 32 & $\begin{array}{c}9.1 \\
8\end{array}$ & 44 & $\begin{array}{c}1.0 \\
4\end{array}$ & 65 & $\begin{array}{c}0.6 \\
2\end{array}$ & 92 & $\begin{array}{c}1.8 \\
6\end{array}$ & 68 & $\begin{array}{c}0.7 \\
9\end{array}$ & 64 & $\begin{array}{c}0.5 \\
9\end{array}$ & & \\
\hline & 0.90 & 1.4 & 36 & 1.5 & 37 & 1.6 & 37 & 1.8 & 37 & 1.8 & 37 & 1.8 & 37 & & \\
\hline & & 44 & 8.6 & 59 & 2.7 & 81 & 1.9 & 08 & 1.1 & 93 & 0.8 & 18 & 0.9 & & \\
\hline & & & 1 & & 9 & & 8 & & 2 & & 2 & & 9 & & \\
\hline & EWM & 1.4 & 37 & 1.5 & 36 & 1.6 & 37 & 1.8 & 37 & 1.9 & 37 & 1.8 & 37 & 0.00 & 3 \\
\hline & A (1.0) & 51 & 3.8 & 63 & 9.1 & 85 & 2.0 & 11 & 0.9 & 07 & 1.4 & 59 & 0.0 & 2706 & 7 \\
\hline & & & 5 & & 0 & & 3 & & 0 & & 0 & & 5 & & \\
\hline & 1.30 & 1.4 & 36 & 1.5 & 37 & 1.6 & 37 & 1.7 & 37 & 1.8 & 37 & 1.8 & 37 & & \\
\hline & & 47 & 8.1 & 49 & 2.7 & 56 & 2.2 & 70 & 1.0 & 80 & 0.0 & 91 & 0.7 & & \\
\hline & & & 0 & & 9 & & 4 & & 9 & & 1 & & 3 & & \\
\hline \multirow[t]{13}{*}{2} & 0.50 & 1.6 & 37 & 1.6 & 37 & 1.7 & 37 & 1.8 & 37 & 1.8 & 37 & 1.7 & 37 & & \\
\hline & & 26 & 0.3 & 94 & 1.3 & 62 & 1.7 & 17 & 1.5 & 08 & 0.2 & 17 & 0.3 & & \\
\hline & & & 0 & & 0 & & 1 & & 2 & & 7 & & 2 & & \\
\hline & 0.60 & 1.6 & 37 & 1.7 & 37 & 1.8 & 37 & 1.8 & 36 & 1.8 & 37 & 1.7 & 37 & & \\
\hline & & 67 & 0.2 & 47 & 0.3 & 27 & 0.6 & 97 & 9.9 & 93 & 0.5 & 46 & 0.5 & & \\
\hline & & & 0 & & 3 & & 0 & & 6 & & 9 & & 3 & & \\
\hline & 0.70 & 1.6 & 37 & 1.7 & 37 & 1.8 & 37 & 1.9 & 37 & 1.9 & 37 & 1.8 & 37 & & \\
\hline & & 98 & 2.8 & 86 & 0.9 & 73 & 0.2 & 53 & 0.0 & 60 & 1.3 & 02 & 0.6 & & \\
\hline & & & 4 & & 4 & & 6 & & 5 & & 4 & & 1 & & \\
\hline & 0.80 & 1.7 & 37 & 1.8 & 37 & 1.9 & 37 & 1.9 & 37 & 2.0 & 37 & 1.8 & 37 & & \\
\hline & & 21 & 1.3 & 13 & 1.7 & 07 & 0.8 & 89 & 0.1 & 06 & 0.3 & 56 & 0.9 & & \\
\hline & & & 4 & & 4 & & 5 & & 6 & & 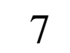 & & 0 & & \\
\hline & 0.90 & 1.7 & 37 & 1.8 & 37 & 1.9 & 37 & 2.0 & 37 & 2.0 & 37 & 1.9 & 37 & & \\
\hline
\end{tabular}




\begin{tabular}{|c|c|c|c|c|c|c|c|c|c|c|c|c|c|c|c|}
\hline & & 36 & $\begin{array}{c}0.4 \\
2\end{array}$ & 31 & $\begin{array}{c}0.5 \\
4\end{array}$ & 27 & $\begin{array}{c}0.6 \\
6\end{array}$ & 11 & $\begin{array}{c}1.2 \\
8\end{array}$ & 34 & $\begin{array}{c}0.8 \\
2\end{array}$ & 04 & $\begin{array}{c}0.2 \\
4\end{array}$ & & \\
\hline & EWM & 1.7 & 37 & 1.8 & 36 & 1.9 & 37 & 2.0 & 37 & 2.0 & 37 & 1.9 & 37 & 0.07 & 3 \\
\hline & $\mathrm{A}(\mathbf{1 . 0})$ & 48 & 1.0 & 42 & 9.7 & 35 & 0.8 & 19 & 1.4 & 45 & 0.1 & 44 & 0.6 & 5386 & 7 \\
\hline & & & 3 & & 5 & & 1 & & 4 & & 6 & & 0 & & \\
\hline & 1.30 & 1.7 & 37 & 1.8 & 37 & 1.9 & 36 & 1.9 & 37 & 2.0 & 37 & 1.9 & 37 & & \\
\hline & & 58 & 0.9 & 42 & 1.8 & 23 & 9.5 & 99 & 0.2 & 42 & 0.7 & 91 & 0.3 & & \\
\hline & & & 2 & & 6 & & 9 & & 8 & & 0 & & 4 & & \\
\hline \multirow[t]{21}{*}{3} & 0.50 & 1.7 & 37 & 1.8 & 36 & 1.8 & 37 & 1.9 & 36 & 1.9 & 36 & 1.8 & 37 & & \\
\hline & & 86 & 1.3 & 43 & 9.8 & 99 & 1.0 & 37 & 9.5 & 11 & 9.8 & 07 & 0.5 & & \\
\hline & & & 2 & & 5 & & 8 & & 4 & & 7 & & 9 & & \\
\hline & 0.60 & 1.8 & 37 & 1.8 & 36 & 1.9 & 36 & 2.0 & 37 & 1.9 & 36 & 1.8 & 37 & & \\
\hline & & 23 & 1.6 & 89 & 9.8 & 56 & 9.8 & 09 & 0.3 & 78 & 9.8 & 13 & 0.0 & & \\
\hline & & & 0 & & 2 & & 5 & & 0 & & 5 & & 9 & & \\
\hline & 0.70 & 1.8 & 37 & 1.9 & 36 & 1.9 & 36 & 2.0 & 37 & 2.0 & 37 & 1.8 & 36 & & \\
\hline & & 52 & 2.0 & 25 & 9.9 & 99 & 9.7 & 59 & 0.4 & 33 & 0.3 & 46 & 9.8 & & \\
\hline & & & 1 & & 8 & & 9 & & 1 & & 0 & & 2 & & \\
\hline & 0.80 & 1.8 & 36 & 1.9 & 37 & 2.0 & 37 & 2.0 & 37 & 2.0 & 37 & 1.8 & 37 & & \\
\hline & & 72 & 9.6 & 50 & 0.5 & 28 & 0.8 & 91 & 0.7 & 69 & 0.1 & 92 & 0.2 & & \\
\hline & & & 3 & & 9 & & 3 & & 4 & & 6 & & 3 & & \\
\hline & 0.90 & 1.8 & 37 & 1.9 & 37 & 2.0 & 36 & 2.1 & 37 & 2.0 & 37 & 1.9 & 37 & & \\
\hline & & 89 & 0.2 & 68 & 0.3 & 45 & 9.9 & 08 & 0.0 & 95 & 0.6 & 38 & 0.2 & & \\
\hline & & & 0 & & 3 & & 6 & & 3 & & 3 & & 4 & & \\
\hline & EWM & 1.9 & 37 & 1.9 & 36 & 2.0 & 37 & 2.1 & 37 & 2.1 & 37 & 1.9 & 37 & 0.27 & 3 \\
\hline & $\mathrm{A}(\mathbf{1 . 0})$ & 00 & 0.7 & 77 & 9.5 & 54 & 0.0 & 15 & 0.9 & 10 & 0.7 & 72 & 0.2 & 0712 & 7 \\
\hline & & & 4 & & 2 & & 0 & & 5 & & 4 & & 4 & & 0 \\
\hline & 1.30 & 1.9 & 37 & 1.9 & 36 & 2.0 & 37 & 2.1 & 36 & 2.1 & 37 & 2.0 & 37 & & \\
\hline & & 14 & 0.0 & 82 & 9.5 & 49 & 0.5 & 00 & 9.9 & 11 & 0.5 & 36 & 0.6 & & \\
\hline & & & 3 & & 6 & & 8 & & 4 & & 3 & & 6 & & \\
\hline \multirow[t]{14}{*}{4} & 0.50 & 1.8 & 37 & 1.9 & 37 & 1.9 & 37 & 2.0 & 36 & 1.9 & 37 & 1.8 & 37 & & \\
\hline & & 92 & 0.4 & 38 & 0.2 & 85 & 0.9 & 12 & 9.6 & 72 & 0.0 & 59 & 0.1 & & \\
\hline & & & 8 & & 1 & & 7 & & 4 & & 4 & & 1 & & \\
\hline & 0.60 & 1.9 & 37 & 1.9 & 36 & 2.0 & 37 & 2.0 & 37 & 2.0 & 37 & 1.8 & 37 & & \\
\hline & & 25 & 0.2 & 82 & 9.7 & 38 & 0.8 & 76 & 0.3 & 29 & 0.5 & 55 & 0.2 & & \\
\hline & & & 3 & & 5 & & 7 & & 5 & & 2 & & 0 & & \\
\hline & 0.70 & 1.9 & 37 & 2.0 & 36 & 2.0 & 36 & 2.1 & 36 & 2.0 & 37 & 1.8 & 37 & & \\
\hline & & 52 & 1.0 & 15 & 9.4 & 79 & 9.8 & 22 & 9.6 & 75 & 0.4 & 77 & 0.7 & & \\
\hline & & & 2 & & 1 & & 9 & & 2 & & 9 & & 8 & & \\
\hline & 0.80 & 1.9 & 37 & 2.0 & 37 & 2.1 & 37 & 2.1 & 37 & 2.1 & 37 & 1.9 & 37 & & \\
\hline & & 72 & 1.4 & 40 & 0.2 & 08 & 0.1 & 53 & 0.6 & 07 & 0.2 & 17 & 0.2 & & \\
\hline & & & 4 & & 1 & & 5 & & 7 & & 6 & & 5 & & \\
\hline & 0.90 & 1.9 & 37 & 2.0 & 37 & 2.1 & 36 & 2.1 & 37 & 2.1 & 37 & 1.9 & 37 & & \\
\hline & & 87 & 0.5 & 57 & 0.7 & 23 & 9.2 & 70 & 0.5 & 33 & 0.0 & 56 & 0.1 & & \\
\hline
\end{tabular}




\begin{tabular}{|c|c|c|c|c|c|c|c|c|c|c|c|c|c|c|c|}
\hline & & & 8 & & 8 & & 7 & & 1 & & 4 & & 0 & & \\
\hline & EWM & 1.9 & 36 & 2.0 & 37 & 2.1 & 37 & 2.1 & 36 & 2.1 & 37 & 1.9 & 37 & 0.56 & 3 \\
\hline & $A(1.0)$ & 99 & 9.6 & 67 & 0.0 & 31 & 0.1 & 76 & 9.9 & 43 & 0.7 & 89 & 0.3 & 4345 & 7 \\
\hline & & & 2 & & 4 & & 5 & & 9 & & 0 & & 9 & & \\
\hline & 1.30 & 2.0 & 37 & 2.0 & 36 & 2.1 & 37 & 2.1 & 36 & 2.1 & 36 & 2.0 & 37 & & \\
\hline & & 16 & 1.3 & 74 & 9.5 & 28 & 0.9 & 64 & 9.4 & 56 & 9.8 & 59 & 0.5 & & \\
\hline & & & 4 & & 0 & & 9 & & 2 & & 0 & & 8 & & \\
\hline 5 & 0.50 & 1.9 & 36 & 2.0 & 37 & 2.0 & 37 & 2.0 & 37 & 2.0 & 37 & 1.9 & 37 & & \\
\hline & & 69 & 9.2 & 12 & 0.2 & 52 & 0.4 & 74 & 0.8 & 26 & 0.3 & 10 & 0.0 & & \\
\hline & & & 3 & & 4 & & 1 & & 6 & & 4 & & 7 & & \\
\hline & 0.60 & 2.0 & 37 & 2.0 & 37 & 2.1 & 36 & 2.1 & 36 & 2.0 & 36 & 1.8 & 37 & & \\
\hline & & 01 & 1.5 & 51 & 0.1 & 00 & 9.7 & 28 & 9.5 & 71 & 9.8 & 91 & 0.5 & & \\
\hline & & & 5 & & 9 & & 6 & & 2 & & 2 & & 1 & & \\
\hline & 0.70 & 2.0 & 37 & 2.0 & 37 & 2.1 & 36 & 2.1 & 37 & 2.1 & 37 & 1.9 & 37 & & \\
\hline & & 25 & 1.1 & 82 & 1.7 & 36 & 9.4 & 71 & 0.2 & 10 & 0.9 & 05 & 0.8 & & \\
\hline & & & 1 & & 0 & & 2 & & 5 & & 8 & & 0 & & \\
\hline & 0.80 & 2.0 & 37 & 2.1 & 37 & 2.1 & 37 & 2.2 & 37 & 2.1 & 37 & 1.9 & 37 & & \\
\hline & & 44 & 0.0 & 05 & 0.8 & 63 & 0.1 & 00 & 0.3 & 41 & 0.7 & 41 & 0.5 & & \\
\hline & & & 6 & & 0 & & 3 & & 9 & & 1 & & 2 & & \\
\hline & 0.90 & 2.0 & 37 & 2.1 & 37 & 2.1 & 36 & 2.2 & 37 & 2.1 & 37 & 1.9 & 37 & & \\
\hline & & 60 & 1.5 & 20 & 0.4 & 76 & 9.7 & 16 & 1.1 & 59 & 0.1 & 77 & 0.3 & & \\
\hline & & & 9 & & 2 & & 5 & & 6 & & 8 & & 2 & & \\
\hline & EWM & 2.0 & 37 & 2.1 & 37 & 2.1 & 37 & 2.2 & 37 & 2.1 & 37 & 2.0 & 37 & 0.93 & 3 \\
\hline & $A(1.0)$ & 71 & 0.4 & 32 & 0.5 & 86 & 1.1 & 22 & 0.5 & 75 & 0.6 & 08 & 0.9 & 0615 & 7 \\
\hline & & & 0 & & 4 & & 4 & & 9 & & 0 & & 3 & & 0 \\
\hline & 1.30 & 2.0 & 36 & 2.1 & 37 & 2.1 & 36 & 2.2 & 37 & 2.1 & 36 & 2.0 & 36 & & \\
\hline & & 88 & 9.6 & 41 & 1.1 & 82 & 9.9 & 12 & 0.7 & 87 & 9.7 & 78 & 9.9 & & \\
\hline & & & 3 & & 4 & & 4 & & 3 & & 3 & & 9 & & \\
\hline
\end{tabular}




\subsection{The out-of-control (OOC) performance of the GWMA-TBE chart}

To study the out-of control (OOC) performance of the proposed GWMA-TBE chart, we used all the combinations of the design parameters shown in Table 2; these combinations ensure that the $A R L_{0}$ 's are close to 370 (when $\delta=1$, i.e., no shift occurred) and therefore guarantee that all the charts are at an equal footing. Because we use only a lower control limit (as we are interested in a sustained downward step shift), we only focus on values for $\delta<1$ and we specifically use $\delta=$ $0.975,0.95,0.925,0.9,0.85,0.8,0.7,0.5$ and 0.25 . Also, we do not consider any shifts $\delta<0.25$, i.e., more than a $75 \%$ decrease, as the Shewhart-type charts are well-known to be more efficient than the GWMA and/or EWMA charts for large changes.

The results for the OOC performance comparisons are shown in Tables 3, 4 and 5 for $k=1,2$ and 3 , respectively, and for some combinations of $(q, \alpha, L)$. The results for $k=4,5$ and other combinations of $(q, \alpha, L)$ are not presented here for conciseness, but are available from the authors upon request. Note the following:

i. Each cell in Tables 3, 4 and 5 displays the $A R L$, the standard deviation of the run-length ( $S D R L)$ as well as the $5^{\text {th }}, 25^{\text {th }}, 50^{\text {th }}, 75^{\text {th }}$ and $95^{\text {th }}$ percentiles;

ii. The tables include the results for the EWMA and the Shewhart-type TBE charts. The highlighted columns in the tables display the results for the EWMA chart whereas the columns on the right-hand side display the results for the Shewhart-type chart;

Although these tables are dense and contain lots of information, a quick comparison of the results reveals the following main points: 
i. Both the GWMA-TBE and the EWMA-TBE charts outperform the Shewhart-type TBE chart for all values of $k$ and for all shifts; this includes the scenario where $\theta$ decreases to a quarter of its original value, i.e., when $\delta=0.25$;

ii. The GWMA-TBE can be designed to outperform the EWMA-TBE chart for very small to moderate shifts; this can be done using a suitably selected combination of $(q, \alpha, L)$. For example, consider Table 3 with the results for $k=1$ and focus on the section where $q=0.8$ : The three GWMA charts with $\alpha=0.5,0.7$ and 0.9 all outperform the EWMA chart when $\alpha=1$. The same is true for other values of $k$ and $(q, \alpha, L)$. This clearly illustrates the benefit of the additional design parameter $(\alpha)$ in constructing a GWMA control chart;

iii. As the value of $k$ increases, the performance for both the GWMA-TBE and EWMA-TBE charts improves. For instance, when $k=1$ and $\delta=0.9$, the $A R L_{1}$ for a GWMA-TBE chart with $q=0.9, \alpha=0.7, L=1.806$ is 125.37 while the $A R L_{1}$ for GWMA-TBE chart when $k=2$ and $\delta=0.9$ and $q=0.9, \alpha=0.7, L=1.960$ is 93.96 . This result indicates that a higher value of $k$ improves the sensitivity of the GWMA and EWMA charts. However, caution should be applied when implementing these charts in practice since a larger value of $k$ also implies more observations/failures has to be collected before a decision can be made about the status of the process. The specific choice of $k$ is left to the practitioners;

iv. As mentioned earlier, the optimal design of the GWMA-TBE chart typically consists of specifying the desired $A R L_{0}$ and $A R L_{1}$ values as well as the magnitude of the process shift ( $\delta$ ) that is anticipated and then select the $(q, \alpha, L)$ combination that provides the desired $A R L$ performance. However, Chan and Zang (2000) argued that it is also important to take 
Table 3: IC and OOC ARL, SDRL as well as the $5^{\text {th }}, \mathbf{2 5}^{\text {th }}, \mathbf{5 0 ^ { \text { th } }}$ (or $\left.M D R L\right), 7^{\text {th }}$ and $95^{\text {th }}$ percentiles of the run-length for different combinations of $(q, \alpha, L)$ when $k=1$.

\begin{tabular}{|c|c|c|c|c|c|c|c|c|c|c|c|c|c|c|c|c|}
\hline & \multicolumn{5}{|c|}{$q=0.8$} & \multicolumn{5}{|c|}{$q=0.9$} & \multicolumn{5}{|c|}{$q=0.95$} & \multirow{2}{*}{$\begin{array}{c}q= \\
0 \\
\alpha= \\
1\end{array}$} \\
\hline & $\begin{array}{l}\alpha= \\
0.5\end{array}$ & 0.7 & $\mathbf{0 . 9}$ & $\begin{array}{c}\alpha \\
=1\end{array}$ & 1.3 & 0.5 & 0.7 & $\mathbf{0 . 9}$ & $\begin{array}{c}\alpha \\
=1\end{array}$ & 1.3 & 0.5 & 0.7 & $\mathbf{0 . 9}$ & $\begin{array}{c}\alpha \\
=1\end{array}$ & 1.3 & \\
\hline Sh & $L=$ & 1.7 & 1.8 & 1.8 & 1.7 & 1.6 & 1.8 & 1.8 & 1.9 & 1.8 & 1.5 & 1.6 & 1.8 & 1.8 & 1.8 & 0.00 \\
\hline ift & $\begin{array}{c}1.59 \\
4\end{array}$ & 5 & 08 & 11 & 7 & 2 & 06 & 93 & 07 & 8 & 52 & 89 & 18 & 59 & 91 & $\begin{array}{c}270 \\
6\end{array}$ \\
\hline \multirow[t]{15}{*}{1} & 371. & 37 & 37 & 37 & 37 & 37 & 37 & 37 & 37 & 37 & 37 & 37 & 37 & 37 & 37 & 370. \\
\hline & 19 & 0.0 & 1.1 & 0.9 & 1.0 & 1.3 & 0.9 & 0.8 & 1.4 & 0.0 & 0.3 & 0.5 & 0.9 & 0.0 & 0.7 & 00 \\
\hline & & 6 & 2 & 0 & 9 & 4 & 6 & 2 & $\mathbf{0}$ & 1 & 4 & 5 & 9 & 5 & 3 & \\
\hline & 377. & 35 & 35 & 35 & 35 & 40 & 35 & 35 & 35 & 35 & 45 & 39 & 36 & 35 & 36 & 369. \\
\hline & & 3.9 & 7.2 & 9.1 & 7.7 & 5.9 & 6.7 & 3.6 & 2.7 & 9.7 & 7.5 & 1.5 & 2.9 & 6.9 & 0.3 & 50 \\
\hline & & $\mathbf{0}$ & 8 & 2 & 9 & 6 & ( & 2 & 8 & 4 & $\mathbf{0}$ & 2 & 7 & 7 & 6 & \\
\hline & 28 & 28 & 27 & 26 & 24 & 28 & 30 & 29 & 28 & 26 & 25 & 30 & 32 & 31 & 29 & 19 \\
\hline & 104 & 11 & 11 & 11 & 11 & 95 & 11 & 11 & 11 & 11 & 82 & 98 & 11 & 11 & 11 & 106 \\
\hline & & 4 & 3 & 2 & 3 & & 3 & 7 & 6 & 2 & & & 0 & 3 & 2 & \\
\hline & 251 & 26 & 26 & 25 & 26 & 23 & 26 & 26 & 26 & 26 & 20 & 23 & 25 & 25 & 26 & 256 \\
\hline & & 3 & 1 & 9 & 1 & 2 & 1 & 4 & 6 & 0 & 1 & 8 & 5 & 8 & 0 & \\
\hline & 501 & 50 & 51 & 50 & 51 & 49 & 50 & 50 & 50 & 50 & 47 & 50 & 50 & 50 & 50 & 512 \\
\hline & & 9 & 2 & 9 & 7 & 7 & 9 & 5 & 9 & 6 & 2 & 5 & 9 & 4 & 7 & \\
\hline & 1144 & 10 & 11 & 11 & 10 & 12 & 11 & 10 & 11 & 11 & 13 & 11 & 11 & 11 & 10 & 110 \\
\hline & & 94 & 00 & 09 & 92 & 04 & 11 & 94 & 00 & 10 & 21 & 58 & 01 & 01 & 85 & 7 \\
\hline \multirow{14}{*}{$\begin{array}{c}\mathbf{0 .} \\
97 \\
5\end{array}$} & 273. & 28 & 30 & 31 & 31 & 25 & 27 & 28 & 29 & 31 & 23 & 25 & 27 & 28 & 30 & 360. \\
\hline & 18 & 8.1 & 2.9 & 0.0 & 8.4 & 3.7 & 0.1 & 7.2 & 5.7 & 1.8 & 6.1 & 3.2 & 3.7 & 3.8 & 3.1 & 76 \\
\hline & & 1 & 3 & 4 & 3 & 2 & 2 & 2 & 2 & 9 & 3 & 8 & 9 & 5 & 2 & \\
\hline & 269. & 28 & 29 & 30 & 31 & 26 & 25 & 27 & 28 & 30 & 26 & 25 & 26 & 27 & 29 & 360. \\
\hline & 29 & 1.9 & 4.9 & 3.0 & 1.1 & 0.5 & 9.6 & 9.3 & 5.9 & 5.3 & 4.2 & 3.1 & 0.4 & 1.1 & 1.3 & 26 \\
\hline & & 6 & 5 & 2 & 2 & 9 & 3 & 3 & & 6 & 8 & 0 & 3 & 7 & 0 & \\
\hline & 25 & 25 & 23 & 22 & 21 & 25 & 27 & 26 & 25 & 23 & 23 & 27 & 29 & 28 & 26 & 18 \\
\hline & 85 & 89 & 91 & 92 & 94 & 75 & 85 & 90 & 92 & 92 & 66 & 78 & 88 & 91 & 94 & 104 \\
\hline & 188 & 20 & 21 & 21 & 22 & 16 & 18 & 20 & 20 & 21 & 14 & 17 & 19 & 20 & 21 & 250 \\
\hline & & 2 & 4 & 7 & 5 & 7 & 9 & 1 & 6 & 7 & 6 & 0 & 2 & 1 & 2 & \\
\hline & 372 & 39 & 41 & 42 & 43 & 34 & 37 & 39 & 40 & 43 & 30 & 34 & 37 & 38 & 41 & 499 \\
\hline & & 4 & 8 & S & 8 & 2 & 1 & 3 & 8 & 0 & 6 & 1 & 4 & 9 & 7 & \\
\hline & 806 & 85 & 87 & 91 & 93 & 77 & 79 & 83 & 86 & 91 & 75 & 76 & 80 & 82 & 89 & 107 \\
\hline & & 1 & 9 & 0 & 9 & 8 & 4 & 6 & 3 & 9 & 7 & 5 & 6 & 5 & 1 & 9 \\
\hline \begin{tabular}{|l}
$\mathbf{0}$ \\
\end{tabular} & 207. & 22 & 25 & 25 & 27 & 18 & 20 & 22 & 23 & 26 & 16 & 18 & 20 & 21 & 24 & 351. \\
\hline 95 & 90 & 8.4 & 0.8 & 9.7 & 9.5 & 4.3 & 2.6 & 5.5 & 8.4 & 0.3 & 7.9 & 5.3 & 7.8 & 9.0 & 4.8 & 53 \\
\hline
\end{tabular}









\begin{tabular}{|c|c|c|c|c|c|c|c|c|c|c|c|c|c|c|c|c|}
\hline & $\begin{array}{r}68.9 \\
0\end{array}$ & $\begin{array}{r}88 . \\
18\end{array}$ & $\begin{array}{r}11 \\
2.3 \\
2\end{array}$ & $\begin{array}{r}12 \\
3.6 \\
4\end{array}$ & $\begin{array}{r}15 \\
5.8 \\
9\end{array}$ & $\begin{array}{r}56 . \\
69\end{array}$ & $\begin{array}{r}65 . \\
30\end{array}$ & $\begin{array}{r}82 . \\
70\end{array}$ & $\begin{array}{r}93 . \\
15\end{array}$ & $\begin{array}{r}12 \\
5.7 \\
2\end{array}$ & $\begin{array}{r}50 . \\
39\end{array}$ & $\begin{array}{r}54 . \\
68\end{array}$ & $\begin{array}{r}66 . \\
51\end{array}$ & $\begin{array}{r}72 . \\
52\end{array}$ & $\begin{array}{r}99 . \\
40\end{array}$ & $\begin{array}{r}314 . \\
07\end{array}$ \\
\hline & 16 & 16 & 15 & 14 & 13 & 17 & 18 & 17 & 16 & 14 & 16 & 19 & 20 & 19 & 16 & 16 \\
\hline & 38 & 39 & 42 & 44 & 50 & 35 & 38 & 38 & 39 & 44 & 32 & 36 & 39 & 38 & 40 & 90 \\
\hline & 67 & 74 & 88 & 95 & $\begin{array}{r}11 \\
3\end{array}$ & 60 & 65 & 72 & 78 & 96 & 54 & 59 & 67 & 68 & 80 & 218 \\
\hline & 116 & $\begin{array}{r}13 \\
5\end{array}$ & $\begin{array}{r}16 \\
8\end{array}$ & $\begin{array}{r}18 \\
2\end{array}$ & $\begin{array}{r}22 \\
2\end{array}$ & $\begin{array}{r}10 \\
0\end{array}$ & $\begin{array}{r}11 \\
0\end{array}$ & $\begin{array}{r}12 \\
9\end{array}$ & $\begin{array}{r}14 \\
2\end{array}$ & $\begin{array}{r}18 \\
3\end{array}$ & 89 & 97 & $\begin{array}{r}11 \\
3\end{array}$ & $\begin{array}{r}11 \\
9\end{array}$ & $\begin{array}{r}15 \\
0\end{array}$ & 435 \\
\hline & 222 & $\begin{array}{r}27 \\
5\end{array}$ & $\begin{array}{r}34 \\
8\end{array}$ & $\begin{array}{r}38 \\
4\end{array}$ & $\begin{array}{r}46 \\
2\end{array}$ & $\begin{array}{r}18 \\
6\end{array}$ & $\begin{array}{r}21 \\
3\end{array}$ & $\begin{array}{r}26 \\
0\end{array}$ & $\begin{array}{r}29 \\
1\end{array}$ & $\begin{array}{r}38 \\
6\end{array}$ & $\begin{array}{r}16 \\
7\end{array}$ & $\begin{array}{r}18 \\
3\end{array}$ & $\begin{array}{r}21 \\
9\end{array}$ & $\begin{array}{r}23 \\
4\end{array}$ & $\begin{array}{r}30 \\
9\end{array}$ & 941 \\
\hline \multirow[t]{7}{*}{$\begin{array}{l}0 . \\
8\end{array}$} & $\begin{array}{r}62.6 \\
8\end{array}$ & $\begin{array}{c}70 . \\
97\end{array}$ & $\begin{array}{r}87 . \\
16\end{array}$ & $\begin{array}{r}95 . \\
27\end{array}$ & $\begin{array}{r}12 \\
2.3 \\
8\end{array}$ & $\begin{array}{r}55 . \\
39\end{array}$ & $\begin{array}{r}60 . \\
14\end{array}$ & $\begin{array}{r}68 . \\
47\end{array}$ & $\begin{array}{r}74 . \\
44\end{array}$ & $\begin{array}{r}96 . \\
27\end{array}$ & $\begin{array}{r}50 . \\
15\end{array}$ & $\begin{array}{r}54 . \\
54\end{array}$ & $\begin{array}{r}60 . \\
23\end{array}$ & $\begin{array}{r}63 . \\
69\end{array}$ & $\begin{array}{r}78 . \\
05\end{array}$ & $\begin{array}{r}296 . \\
10\end{array}$ \\
\hline & $\begin{array}{r}46.1 \\
1\end{array}$ & $\begin{array}{r}58 . \\
20\end{array}$ & $\begin{array}{r}78 . \\
69\end{array}$ & $\begin{array}{r}86 . \\
64\end{array}$ & $\begin{array}{r}11 \\
6.8 \\
7 \\
\end{array}$ & $\begin{array}{r}37 . \\
46\end{array}$ & $\begin{array}{r}43 . \\
04\end{array}$ & $\begin{array}{r}55 . \\
89\end{array}$ & $\begin{array}{r}63 . \\
49\end{array}$ & $\begin{array}{r}87 . \\
90\end{array}$ & $\begin{array}{r}33 . \\
09\end{array}$ & $\begin{array}{r}35 . \\
16\end{array}$ & $\begin{array}{r}42 . \\
16\end{array}$ & $\begin{array}{r}47 . \\
98\end{array}$ & $\begin{array}{r}68 . \\
62\end{array}$ & $\begin{array}{r}295 . \\
60\end{array}$ \\
\hline & 14 & 14 & 13 & 12 & 11 & 15 & 16 & 15 & 14 & 12 & 14 & 17 & 17 & 17 & 14 & 15 \\
\hline & 30 & 30 & 32 & 34 & 39 & 29 & 30 & 30 & 30 & 34 & 26 & 30 & 31 & 30 & 30 & 85 \\
\hline & 50 & 53 & 62 & 68 & 86 & 46 & 48 & 52 & 55 & 69 & 42 & 45 & 48 & 50 & 57 & 205 \\
\hline & 83 & 93 & $\begin{array}{r}11 \\
7 \\
\end{array}$ & $\begin{array}{r}13 \\
0 \\
\end{array}$ & $\begin{array}{r}16 \\
6 \\
\end{array}$ & 72 & 78 & 89 & 98 & $\begin{array}{r}13 \\
1 \\
\end{array}$ & 64 & 70 & 77 & 82 & $\begin{array}{r}10 \\
3 \\
\end{array}$ & 410 \\
\hline & 152 & $\begin{array}{r}18 \\
4\end{array}$ & $\begin{array}{r}24 \\
4\end{array}$ & $\begin{array}{r}26 \\
9\end{array}$ & $\begin{array}{r}36 \\
0\end{array}$ & $\begin{array}{r}12 \\
9\end{array}$ & $\begin{array}{r}14 \\
5\end{array}$ & $\begin{array}{r}17 \\
7\end{array}$ & $\begin{array}{r}20 \\
0\end{array}$ & $\begin{array}{r}27 \\
1\end{array}$ & $\begin{array}{r}11 \\
6\end{array}$ & $\begin{array}{r}12 \\
4\end{array}$ & $\begin{array}{r}14 \\
4\end{array}$ & $\begin{array}{r}15 \\
8\end{array}$ & $\begin{array}{r}21 \\
2\end{array}$ & 886 \\
\hline \multirow[t]{7}{*}{$\begin{array}{l}0 . \\
7\end{array}$} & $\begin{array}{r}37.0 \\
0 \\
\end{array}$ & $\begin{array}{r}39 . \\
70\end{array}$ & $\begin{array}{r}46 . \\
70\end{array}$ & $\begin{array}{r}51 . \\
79\end{array}$ & $\begin{array}{r}69 . \\
10\end{array}$ & $\begin{array}{r}33 . \\
98\end{array}$ & $\begin{array}{r}35 . \\
55\end{array}$ & $\begin{array}{r}38 . \\
09\end{array}$ & $\begin{array}{r}40 . \\
30\end{array}$ & $\begin{array}{r}51 . \\
45\end{array}$ & $\begin{array}{r}31 . \\
51\end{array}$ & $\begin{array}{r}33 . \\
79\end{array}$ & $\begin{array}{r}35 . \\
60\end{array}$ & $\begin{array}{r}36 . \\
55\end{array}$ & $\begin{array}{r}41 . \\
68\end{array}$ & $\begin{array}{r}259 . \\
15\end{array}$ \\
\hline & $\begin{array}{r}22.5 \\
9\end{array}$ & $\begin{array}{r}27 . \\
46\end{array}$ & $\begin{array}{r}37 . \\
54\end{array}$ & $\begin{array}{r}43 . \\
80\end{array}$ & $\begin{array}{r}62 . \\
94\end{array}$ & $\begin{array}{r}18 . \\
95\end{array}$ & $\begin{array}{r}20 . \\
40\end{array}$ & $\begin{array}{r}25 . \\
55\end{array}$ & $\begin{array}{r}29 . \\
19\end{array}$ & $\begin{array}{r}44 . \\
11\end{array}$ & $\begin{array}{r}17 . \\
01\end{array}$ & $\begin{array}{r}17 . \\
44\end{array}$ & $\begin{array}{r}19 . \\
51\end{array}$ & $\begin{array}{r}21 . \\
71\end{array}$ & $\begin{array}{r}31 . \\
13\end{array}$ & $\begin{array}{r}258 . \\
65\end{array}$ \\
\hline & 12 & 11 & 10 & 9 & 9 & 12 & 13 & 12 & 12 & 10 & 12 & 14 & 14 & 14 & 12 & 13 \\
\hline & 20 & 20 & 20 & 21 & 24 & 20 & 21 & 20 & 20 & 20 & 19 & 21 & 22 & 21 & 20 & 74 \\
\hline & 32 & 33 & 36 & 38 & 50 & 30 & 31 & 31 & 32 & 38 & 28 & 30 & 31 & 31 & 32 & 179 \\
\hline & 47 & 51 & 61 & 69 & 94 & 43 & 45 & 48 & 51 & 68 & 40 & 42 & 44 & 45 & 53 & 359 \\
\hline & 82 & 94 & $\begin{array}{r}12 \\
1 \\
\end{array}$ & $\begin{array}{r}13 \\
8 \\
\end{array}$ & $\begin{array}{r}19 \\
7 \\
\end{array}$ & 71 & 76 & 89 & 98 & $\begin{array}{r}13 \\
8\end{array}$ & 64 & 67 & 74 & 79 & $\begin{array}{r}10 \\
4\end{array}$ & 775 \\
\hline \multirow[t]{7}{*}{$\begin{array}{l}0 . \\
5\end{array}$} & $\begin{array}{r}17.2 \\
6 \\
\end{array}$ & $\begin{array}{r}17 . \\
05\end{array}$ & $\begin{array}{r}17 . \\
65\end{array}$ & $\begin{array}{r}18 . \\
47\end{array}$ & $\begin{array}{r}23 . \\
36\end{array}$ & $\begin{array}{r}16 . \\
91\end{array}$ & $\begin{array}{r}17 . \\
29\end{array}$ & $\begin{array}{r}16 . \\
90\end{array}$ & $\begin{array}{r}16 . \\
84\end{array}$ & $\begin{array}{r}18 . \\
18\end{array}$ & $\begin{array}{r}16 . \\
23\end{array}$ & $\begin{array}{r}17 . \\
55\end{array}$ & $\begin{array}{r}17 . \\
92\end{array}$ & $\begin{array}{r}17 . \\
64\end{array}$ & $\begin{array}{r}16 . \\
87\end{array}$ & $\begin{array}{r}185 . \\
25 \\
\end{array}$ \\
\hline & 7.14 & $\begin{array}{r}7.6 \\
3\end{array}$ & $\begin{array}{r}9.7 \\
6\end{array}$ & $\begin{array}{r}11 . \\
43\end{array}$ & $\begin{array}{r}18 . \\
50\end{array}$ & $\begin{array}{r}6.3 \\
0\end{array}$ & $\begin{array}{r}6.2 \\
2\end{array}$ & $\begin{array}{r}6.6 \\
0\end{array}$ & $\begin{array}{r}7.3 \\
2\end{array}$ & $\begin{array}{r}11 . \\
17\end{array}$ & $\begin{array}{r}5.8 \\
2\end{array}$ & $\begin{array}{r}5.6 \\
0\end{array}$ & $\begin{array}{r}5.6 \\
4\end{array}$ & $\begin{array}{r}5.7 \\
7\end{array}$ & $\begin{array}{r}7.3 \\
2\end{array}$ & $\begin{array}{r}184 . \\
75\end{array}$ \\
\hline & 8 & 8 & 7 & 7 & 6 & 9 & 10 & 9 & 9 & 7 & 9 & 10 & 11 & 11 & 9 & 9 \\
\hline & 12 & 12 & 11 & 11 & 10 & 12 & 13 & 12 & 12 & 10 & 12 & 13 & 14 & 13 & 12 & 53 \\
\hline & 16 & 15 & 15 & 15 & 17 & 16 & 16 & 15 & 15 & 15 & 15 & 17 & 17 & 16 & 15 & 128 \\
\hline & 21 & 21 & 22 & 23 & 30 & 20 & 21 & 20 & 20 & 22 & 19 & 21 & 21 & 21 & 20 & 256 \\
\hline & 31 & 32 & 37 & 41 & 60 & 29 & 29 & 30 & 31 & 40 & 27 & 28 & 28 & 28 & 31 & 553 \\
\hline
\end{tabular}




\begin{tabular}{|c|r|r|r|r|r|r|r|r|r|r|r|r|r|r|r|r|}
\hline $\begin{array}{r}\text { 0. } \\
25\end{array}$ & $\mathbf{8 . 8 9}$ & $\mathbf{8 . 5}$ & $\mathbf{7 . 9}$ & $\mathbf{7 . 7}$ & $\mathbf{7 . 4}$ & $\mathbf{9 . 1}$ & $\mathbf{9 . 5}$ & $\mathbf{9 . 1}$ & $\mathbf{8 . 8}$ & $\mathbf{7 . 8}$ & $\mathbf{9 . 0}$ & $\mathbf{1 0 .}$ & $\mathbf{1 0 .}$ & $\mathbf{1 0 .}$ & $\mathbf{9 . 2}$ & $\mathbf{9 2 . 8}$ \\
& & $\mathbf{3}$ & $\mathbf{7}$ & $\mathbf{2}$ & $\mathbf{5}$ & $\mathbf{9}$ & $\mathbf{5}$ & $\mathbf{8}$ & $\mathbf{5}$ & $\mathbf{9}$ & $\mathbf{5}$ & $\mathbf{1 8}$ & $\mathbf{5 8}$ & $\mathbf{4 0}$ & $\mathbf{3}$ & $\mathbf{8}$ \\
\cline { 2 - 15 } & $\mathbf{2 . 0 4}$ & $\mathbf{1 . 8}$ & $\mathbf{1 . 9}$ & $\mathbf{2 . 0}$ & $\mathbf{2 . 7}$ & $\mathbf{1 . 8}$ & $\mathbf{1 . 6}$ & $\mathbf{1 . 5}$ & $\mathbf{1 . 5}$ & $\mathbf{1 . 6}$ & $\mathbf{1 . 7}$ & $\mathbf{1 . 5}$ & $\mathbf{1 . 4}$ & $\mathbf{1 . 3}$ & $\mathbf{1 . 2}$ & $\mathbf{9 2 . 3}$ \\
& & $\mathbf{9}$ & $\mathbf{1}$ & $\mathbf{1}$ & $\mathbf{5}$ & $\mathbf{8}$ & $\mathbf{9}$ & $\mathbf{7}$ & $\mathbf{4}$ & $\mathbf{8}$ & $\mathbf{5}$ & $\mathbf{8}$ & $\mathbf{5}$ & $\mathbf{9}$ & $\mathbf{8}$ & $\mathbf{7}$ \\
\hline & 6 & 6 & 6 & 5 & 5 & 7 & 7 & 7 & 7 & 6 & 7 & 8 & 9 & 9 & 8 & 5 \\
\hline & 7 & 7 & 7 & 6 & 6 & 8 & 8 & 8 & 8 & 7 & 8 & 9 & 10 & 9 & 8 & 27 \\
\hline & 9 & 8 & 8 & 7 & 7 & 9 & 9 & 9 & 9 & 7 & 9 & 10 & 10 & 10 & 9 & 64 \\
\hline & 10 & 10 & 9 & 9 & 8 & 10 & 10 & 10 & 10 & 9 & 10 & 11 & 11 & 11 & 10 & 128 \\
\hline & 13 & 12 & 12 & 12 & 13 & 13 & 13 & 12 & 12 & 11 & 12 & 13 & 13 & 13 & 12 & 277 \\
\hline
\end{tabular}


Table 4: IC and OOC ARL, SDRL as well as the $5^{\text {th }}, 25^{\text {th }}, \mathbf{5 0 ^ { \text { th } }}$ (or $\left.M D R L\right), 75^{\text {th }}$ and $95^{\text {th }}$ percentiles of the run-length for different combinations of $(q, \alpha, L)$ when $k=2$.

\begin{tabular}{|c|c|c|c|c|c|c|c|c|c|c|c|c|c|c|c|c|}
\hline & \multicolumn{5}{|c|}{$q=\mathbf{0 . 8}$} & \multicolumn{5}{|c|}{$q=0.9$} & \multicolumn{5}{|c|}{$q=0.95$} & \multirow{2}{*}{$\begin{array}{c}q= \\
0 \\
\alpha= \\
1\end{array}$} \\
\hline & $\begin{array}{l}\alpha= \\
0.5\end{array}$ & 0.7 & 0.9 & $\begin{array}{c}\alpha \\
=1\end{array}$ & $\mathbf{1 . 3}$ & 0.5 & 0.7 & 0.9 & $\begin{array}{c}\alpha \\
=1\end{array}$ & 1.3 & 0.5 & 0.7 & 0.9 & $\begin{array}{c}\alpha \\
=1\end{array}$ & 1.3 & \\
\hline Sh & $L=$ & 1.9 & 2.0 & 2.0 & 1.9 & 1.8 & 1.9 & 2.0 & 2.0 & 2.0 & 1.7 & 1.8 & 1.9 & 1.9 & 1.9 & 0.07 \\
\hline ift & $\begin{array}{c}1.81 \\
7\end{array}$ & 53 & 11 & 19 & 99 & 08 & 6 & 34 & 45 & 42 & 17 & 02 & 04 & 44 & 91 & $\begin{array}{c}538 \\
6\end{array}$ \\
\hline \multirow[t]{15}{*}{1} & 371. & 37 & 37 & 37 & 37 & 37 & 37 & 37 & 37 & 37 & 37 & 37 & 37 & 37 & 37 & 370. \\
\hline & 52 & 0.0 & 1.2 & 1.4 & 0.2 & 0.2 & 1.3 & 0.8 & 0.1 & 0.7 & 0.3 & 0.6 & 0.2 & 0.6 & 0.3 & 00 \\
\hline & & 5 & 8 & 4 & 8 & 7 & 4 & 2 & 6 & $\mathbf{0}$ & 2 & 1 & 4 & 0 & 4 & \\
\hline & 378. & 36 & 36 & 36 & 36 & 39 & 36 & 35 & 35 & 35 & 44 & 38 & 35 & 35 & 35 & 369. \\
\hline & & 0.3 & 1.1 & 0.6 & 0.6 & 6.9 & 5.6 & 6.4 & 4.3 & 9.0 & 4.3 & 2.3 & 8.3 & 3.3 & 0.5 & 50 \\
\hline & & 8 & 8 & 6 & 1 & 4 & 7 & 4 & 3 & 3 & 5 & 8 & 6 & 0 & 8 & \\
\hline & 27 & 27 & 26 & 25 & 24 & 26 & 30 & 29 & 28 & 27 & 24 & 29 & 31 & 31 & 28 & 19 \\
\hline & 106 & 11 & 11 & 11 & 11 & 97 & 11 & 11 & 11 & 11 & 83 & 10 & 11 & 11 & 11 & 106 \\
\hline & & 2 & 2 & 1 & 0 & & 0 & 6 & 5 & 3 & & 1 & 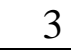 & 7 & 6 & \\
\hline & 251 & 25 & 25 & 25 & 25 & 23 & 25 & 25 & 25 & 26 & 21 & 24 & 25 & 26 & 26 & 256 \\
\hline & & 8 & 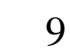 & 9 & 8 & 7 & 5 & 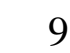 & 9 & 0 & 0 & 6 & 9 & 0 & 2 & \\
\hline & 509 & 51 & 51 & 51 & 51 & 50 & 51 & 51 & 51 & 51 & 48 & 50 & 51 & 51 & 51 & 512 \\
\hline & & 2 & 3 & 1 & 3 & 3 & 3 & 3 & 5 & 1 & 2 & 5 & 2 & 4 & 7 & \\
\hline & 1122 & 10 & 10 & 11 & 10 & 11 & 10 & 10 & 10 & 10 & 12 & 11 & 10 & 10 & 10 & 110 \\
\hline & & 86 & 97 & 14 & 96 & 64 & 99 & 84 & 80 & 95 & 67 & 41 & 85 & 74 & 72 & 7 \\
\hline \multirow{14}{*}{$\begin{array}{c}\text { 0. } \\
97 \\
5\end{array}$} & 250. & 26 & 28 & 29 & 30 & 22 & 24 & 26 & 27 & 28 & 20 & 22 & 24 & 25 & 27 & 352. \\
\hline & 45 & 7.0 & 5.2 & 1.6 & 0.3 & 6.4 & 5.8 & 4.3 & 0.6 & 8.4 & 7.6 & 8.8 & 6.1 & 6.1 & 5.7 & 18 \\
\hline & & 9 & 7 & 2 & 9 & 6 & 1 & 3 & 1 & 9 & 0 & 7 & 2 & 5 & 1 & \\
\hline & 241. & 25 & 27 & 28 & 29 & 22 & 23 & 24 & 25 & 27 & 21 & 22 & 23 & 24 & 26 & 351. \\
\hline & 42 & 7.5 & 7.9 & 3.2 & 2.0 & 4.3 & 2.4 & 9.8 & 6.4 & 7.6 & 8.1 & 3.4 & 2.8 & 0.4 & 2.9 & 68 \\
\hline & & 7 & 9 & 4 & 9 & 6 & 8 & 9 & 3 & 6 & 3 & 3 & 6 & 3 & 5 & \\
\hline & 22 & 22 & 22 & 21 & 21 & 22 & 25 & 24 & 23 & 22 & 20 & 25 & 26 & 26 & 23 & 18 \\
\hline & 79 & 83 & 86 & 86 & 90 & 71 & 81 & 85 & 85 & 87 & 63 & 74 & 81 & 85 & 88 & 101 \\
\hline & 176 & 18 & 20 & 20 & 21 & 15 & 17 & 18 & 19 & 20 & 13 & 15 & 17 & 18 & 19 & 244 \\
\hline & & 7 & 0 & 6 & 3 & 4 & 4 & 6 & 0 & 4 & 5 & 8 & 5 & 3 & 4 & \\
\hline & 346 & 36 & 39 & 40 & 42 & 30 & 33 & 36 & 37 & 39 & 27 & 31 & 33 & 35 & 37 & 488 \\
\hline & & 7 & 1 & 4 & 0 & 8 & 9 & 3 & 4 & 8 & 5 & 0 & 6 & 2 & 9 & \\
\hline & 728 & 78 & 84 & 86 & 86 & 66 & 70 & 77 & 79 & 85 & 63 & 67 & 71 & 74 & 81 & 105 \\
\hline & & 3 & 7 & 4 & 9 & 7 & 9 & 4 & 1 & 5 & 5 & 1 & 3 & 2 & 0 & 4 \\
\hline $\mathbf{0}$. & 177. & 19 & 22 & 22 & 24 & 15 & 17 & 19 & 20 & 22 & 13 & 15 & 17 & 18 & 20 & 334. \\
\hline 95 & 21 & 6.5 & 0.9 & 8.9 & 8.4 & 3.3 & 0.7 & 1.8 & 1.2 & 8.0 & 6.5 & 2.0 & 0.1 & 1.3 & 5.8 & 80 \\
\hline
\end{tabular}




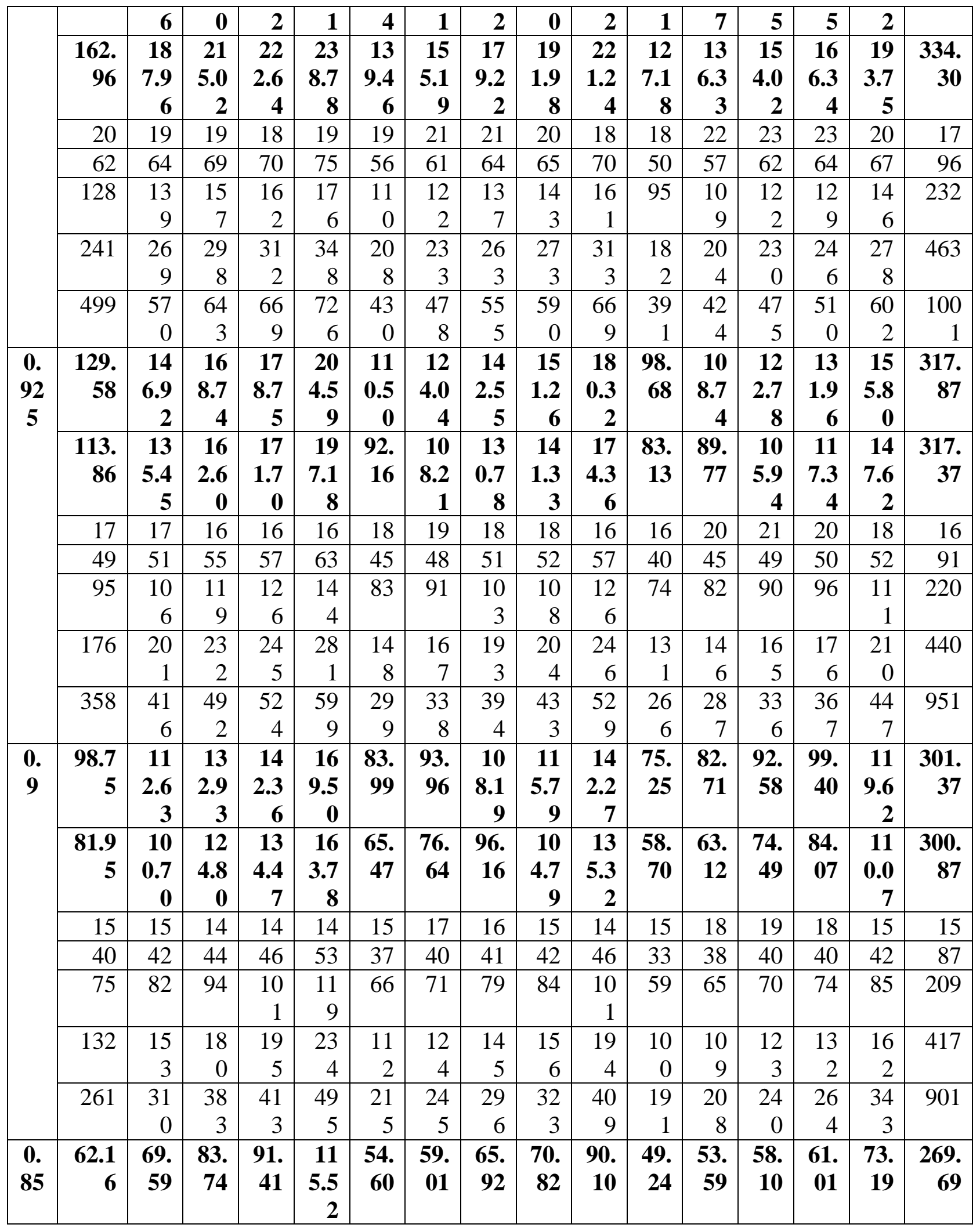




\begin{tabular}{|c|c|c|c|c|c|c|c|c|c|c|c|c|c|c|c|c|}
\hline & $\begin{array}{r}46.7 \\
1\end{array}$ & $\begin{array}{r}57 . \\
74\end{array}$ & $\begin{array}{r}75 . \\
42\end{array}$ & $\begin{array}{r}83 . \\
50\end{array}$ & $\begin{array}{r}10 \\
9.7 \\
9 \\
\end{array}$ & $\begin{array}{l}37 . \\
84\end{array}$ & $\begin{array}{r}42 . \\
68\end{array}$ & $\begin{array}{r}52 . \\
66\end{array}$ & $\begin{array}{r}59 . \\
46\end{array}$ & $\begin{array}{r}82 . \\
06\end{array}$ & $\begin{array}{r}33 . \\
63\end{array}$ & $\begin{array}{r}35 . \\
61\end{array}$ & $\begin{array}{r}41 . \\
05\end{array}$ & $\begin{array}{r}45 . \\
34\end{array}$ & $\begin{array}{r}63 . \\
12\end{array}$ & $\begin{array}{r}269 \\
19\end{array}$ \\
\hline & 13 & 12 & 11 & 11 & 11 & 13 & 14 & 14 & 13 & 11 & 12 & 15 & 16 & 15 & 13 & 14 \\
\hline & 29 & 29 & 31 & 32 & 37 & 27 & 28 & 29 & 29 & 32 & 25 & 28 & 29 & 29 & 29 & 77 \\
\hline & 50 & 53 & 61 & 65 & 81 & 45 & 47 & 51 & 53 & 65 & 41 & 44 & 46 & 48 & 55 & 187 \\
\hline & 82 & 92 & $\begin{array}{r}11 \\
3\end{array}$ & $\begin{array}{r}12 \\
4\end{array}$ & $\begin{array}{r}15 \\
9\end{array}$ & 72 & 77 & 87 & 95 & $\begin{array}{r}12 \\
1\end{array}$ & 64 & 69 & 75 & 79 & 98 & 373 \\
\hline & 155 & 18 & 23 & 25 & 33 & 12 & 14 & 17 & 19 & 25 & 11 & 12 & 13 & 15 & 19 & 806 \\
\hline & & 5 & 4 & 7 & 2 & 9 & 4 & 3 & 0 & 2 & 6 & 3 & 9 & 3 & 7 & \\
\hline \multirow[t]{7}{*}{$\begin{array}{l}\text { 0. } \\
8\end{array}$} & $\begin{array}{r}43.2 \\
6\end{array}$ & $\begin{array}{r}46 . \\
60\end{array}$ & $\begin{array}{r}55 . \\
11\end{array}$ & $\begin{array}{c}60 . \\
76\end{array}$ & $\begin{array}{c}78 . \\
57\end{array}$ & $\begin{array}{r}38 . \\
45\end{array}$ & $\begin{array}{r}40 . \\
53\end{array}$ & $\begin{array}{r}44 . \\
02\end{array}$ & $\begin{array}{r}46 . \\
63\end{array}$ & $\begin{array}{r}59 . \\
51\end{array}$ & $\begin{array}{r}35 . \\
28\end{array}$ & $\begin{array}{r}37 . \\
84\end{array}$ & $\begin{array}{r}40 . \\
01\end{array}$ & $\begin{array}{r}41 . \\
32\end{array}$ & $\begin{array}{r}47 . \\
92\end{array}$ & $\begin{array}{r}239 . \\
77\end{array}$ \\
\hline & $\begin{array}{r}29.1 \\
3\end{array}$ & $\begin{array}{r}35 . \\
07\end{array}$ & $\begin{array}{r}46 . \\
54\end{array}$ & $\begin{array}{r}53 . \\
34\end{array}$ & $\begin{array}{r}73 . \\
57\end{array}$ & $\begin{array}{r}23 . \\
93\end{array}$ & $\begin{array}{r}25 . \\
78\end{array}$ & $\begin{array}{r}32 . \\
28\end{array}$ & $\begin{array}{r}36 . \\
32\end{array}$ & $\begin{array}{r}51 . \\
58\end{array}$ & $\begin{array}{r}21 . \\
40\end{array}$ & $\begin{array}{r}22 . \\
00\end{array}$ & $\begin{array}{r}24 . \\
41\end{array}$ & $\begin{array}{c}26 . \\
90\end{array}$ & $\begin{array}{r}38 . \\
39\end{array}$ & $\begin{array}{r}239 . \\
27\end{array}$ \\
\hline & 11 & 10 & 10 & 9 & 9 & 11 & 12 & 11 & 11 & 10 & 11 & 13 & 14 & 13 & 11 & 12 \\
\hline & 22 & 22 & 22 & 23 & 27 & 21 & 22 & 21 & 21 & 23 & 20 & 22 & 22 & 22 & 21 & 69 \\
\hline & 36 & 37 & 41 & 44 & 57 & 33 & 34 & 35 & 36 & 44 & 30 & 32 & 34 & 34 & 36 & 166 \\
\hline & 57 & 61 & 73 & 81 & $\begin{array}{r}10 \\
6\end{array}$ & 50 & 53 & 57 & 61 & 80 & 46 & 49 & 51 & 53 & 63 & 332 \\
\hline & 102 & $\begin{array}{r}11 \\
7\end{array}$ & $\begin{array}{r}15 \\
1\end{array}$ & $\begin{array}{r}16 \\
8\end{array}$ & $\begin{array}{r}22 \\
4\end{array}$ & 85 & 91 & $\begin{array}{r}10 \\
8\end{array}$ & $\begin{array}{r}11 \\
8\end{array}$ & $\begin{array}{r}16 \\
3\end{array}$ & 77 & 80 & 88 & 96 & $\begin{array}{r}12 \\
3\end{array}$ & 717 \\
\hline \multirow[t]{7}{*}{$\begin{array}{c}0 . \\
7\end{array}$} & $\begin{array}{r}24.3 \\
9\end{array}$ & $\begin{array}{r}24 . \\
95\end{array}$ & $\begin{array}{r}27 . \\
29\end{array}$ & $\begin{array}{r}29 . \\
16\end{array}$ & $\begin{array}{r}38 . \\
05\end{array}$ & $\begin{array}{r}22 . \\
74\end{array}$ & $\begin{array}{r}23 . \\
40\end{array}$ & $\begin{array}{r}23 . \\
79\end{array}$ & $\begin{array}{r}24 . \\
34\end{array}$ & $\begin{array}{r}28 . \\
47\end{array}$ & $\begin{array}{r}21 . \\
42\end{array}$ & $\begin{array}{r}22 . \\
88\end{array}$ & $\begin{array}{r}23 . \\
52\end{array}$ & $\begin{array}{c}23 . \\
56\end{array}$ & $\begin{array}{r}24 . \\
51\end{array}$ & $\begin{array}{r}185 . \\
22\end{array}$ \\
\hline & $\begin{array}{r}13.6 \\
0\end{array}$ & $\begin{array}{r}15 . \\
28\end{array}$ & $\begin{array}{r}19 . \\
54\end{array}$ & $\begin{array}{r}22 . \\
02\end{array}$ & $\begin{array}{r}33 . \\
10\end{array}$ & $\begin{array}{r}11 . \\
57\end{array}$ & $\begin{array}{r}11 . \\
81\end{array}$ & $\begin{array}{r}13 . \\
39\end{array}$ & $\begin{array}{r}14 . \\
93\end{array}$ & $\begin{array}{r}21 . \\
22\end{array}$ & $\begin{array}{r}10 . \\
58\end{array}$ & $\begin{array}{r}10 . \\
47\end{array}$ & $\begin{array}{r}10 . \\
93\end{array}$ & $\begin{array}{r}11 . \\
53\end{array}$ & $\begin{array}{r}15 . \\
43\end{array}$ & $\begin{array}{r}184 . \\
72\end{array}$ \\
\hline & 8 & 8 & 7 & 7 & 6 & 9 & 9 & 9 & 9 & 7 & 9 & 10 & 11 & 11 & 9 & 9 \\
\hline & 14 & 14 & 13 & 13 & 15 & 14 & 15 & 14 & 14 & 13 & 14 & 15 & 16 & 15 & 14 & 53 \\
\hline & 21 & 21 & 22 & 23 & 28 & 20 & 21 & 20 & 20 & 22 & 19 & 21 & 21 & 21 & 20 & 128 \\
\hline & 31 & 32 & 35 & 38 & 51 & 29 & 29 & 30 & 31 & 37 & 27 & 28 & 29 & 29 & 30 & 256 \\
\hline & 51 & 55 & 66 & 73 & $\begin{array}{r}10 \\
5\end{array}$ & 45 & 46 & 50 & 54 & 71 & 42 & 43 & 44 & 46 & 55 & 553 \\
\hline \multirow[t]{8}{*}{$\begin{array}{c}0 . \\
5\end{array}$} & $\begin{array}{r}11.1 \\
6\end{array}$ & $\begin{array}{r}10 . \\
79\end{array}$ & $\begin{array}{r}10 . \\
53\end{array}$ & $\begin{array}{c}10 . \\
56\end{array}$ & $\begin{array}{c}11 . \\
56\end{array}$ & $\begin{array}{r}11 . \\
06\end{array}$ & $\begin{array}{r}11 . \\
39\end{array}$ & $\begin{array}{r}11 . \\
07\end{array}$ & $\begin{array}{r}10 . \\
77\end{array}$ & $\begin{array}{r}10 . \\
43\end{array}$ & $\begin{array}{r}10 . \\
74\end{array}$ & $\begin{array}{r}11 . \\
79\end{array}$ & $\begin{array}{r}12 . \\
14\end{array}$ & $\begin{array}{r}11 . \\
98\end{array}$ & $\begin{array}{c}10 . \\
96\end{array}$ & $\begin{array}{r}97.2 \\
2\end{array}$ \\
\hline & 4.39 & 4.2 & 4.6 & 5.0 & 7.2 & 3.9 & 3.7 & 3.6 & 3.6 & 4.6 & 3.6 & 3.4 & 3.2 & 3.1 & 3.3 & 96.7 \\
\hline & & 4 & 1 & 3 & 2 & 3 & $\mathbf{0}$ & 3 & 8 & 0 & 8 & 2 & 5 & 9 & 6 & 2 \\
\hline & 6 & 6 & 5 & 5 & 5 & 6 & 7 & 7 & 6 & 6 & 6 & 7 & 8 & 8 & 7 & 5 \\
\hline & 8 & 8 & 7 & 7 & 6 & 8 & 9 & 8 & 8 & 7 & 8 & 9 & 10 & 10 & 9 & 28 \\
\hline & 10 & 10 & 9 & 9 & 9 & 10 & 11 & 10 & 10 & 9 & 10 & 11 & 12 & 11 & 10 & 67 \\
\hline & 14 & 13 & 13 & 13 & 14 & 13 & 13 & 13 & 13 & 12 & 13 & 14 & 14 & 14 & 12 & 134 \\
\hline & 19 & 19 & 19 & 20 & 26 & 18 & 18 & 18 & 18 & 20 & 17 & 18 & 18 & 18 & 17 & 290 \\
\hline \multirow{3}{*}{$\begin{array}{c}0 . \\
25\end{array}$} & 5.58 & 5.5 & 5.2 & 5.0 & 4.7 & 5.8 & 6.2 & 6.2 & 6.0 & 5.5 & 5.7 & 6.7 & 7.3 & 7.3 & 6.7 & 26.8 \\
\hline & & ( & 4 & 8 & 0 & 1 & 8 & 4 & 9 & 7 & 9 & 8 & 1 & 2 & 7 & 2 \\
\hline & 1.24 & 1.0 & 0.9 & 0.9 & 0.9 & 1.1 & 1.0 & 0.8 & 0.8 & 0.7 & 1.1 & 0.9 & 0.8 & 0.8 & 0.7 & 26.3 \\
\hline
\end{tabular}




\begin{tabular}{|r|r|r|r|r|r|r|r|r|r|r|r|r|r|r|r|r|}
\hline & & $\mathbf{8}$ & $\mathbf{9}$ & $\mathbf{6}$ & $\mathbf{5}$ & $\mathbf{7}$ & $\mathbf{0}$ & $\mathbf{9}$ & $\mathbf{4}$ & $\mathbf{4}$ & $\mathbf{1}$ & $\mathbf{6}$ & $\mathbf{5}$ & $\mathbf{0}$ & $\mathbf{1}$ & $\mathbf{2}$ \\
\hline 4 & 4 & 4 & 4 & 4 & 4 & 5 & 5 & 5 & 5 & 4 & 5 & 6 & 6 & 6 & 1 \\
\hline 5 & 5 & 5 & 4 & 4 & 5 & 6 & 6 & 6 & 5 & 5 & 6 & 7 & 7 & 6 & 8 \\
\hline 5 & 5 & 5 & 5 & 4 & 6 & 6 & 6 & 6 & 5 & 6 & 7 & 7 & 7 & 7 & 18 \\
\hline 6 & 6 & 6 & 6 & 5 & 6 & 7 & 7 & 7 & 6 & 6 & 7 & 8 & 8 & 7 & 36 \\
\hline 8 & 7 & 7 & 7 & 6 & 8 & 8 & 8 & 8 & 7 & 8 & 8 & 9 & 9 & 8 & 79 \\
\hline
\end{tabular}


Table 5: IC and OOC $A R L, S D R L$ as well as the $5^{\text {th }}, 25^{\text {th }}, 50^{\text {th }}($ or $M D R L), 75^{\text {th }}$ and $95^{\text {th }}$ percentiles of the run-length for different combinations of $(q, \alpha, L)$ when $k=3$.

\begin{tabular}{|c|c|c|c|c|c|c|c|c|c|c|c|c|c|c|c|c|}
\hline & \multicolumn{5}{|c|}{$q=\mathbf{0 . 8}$} & \multicolumn{5}{|c|}{$q=0.9$} & \multicolumn{5}{|c|}{$q=0.95$} & \multirow{2}{*}{$\begin{array}{c}q= \\
0 \\
\alpha= \\
1\end{array}$} \\
\hline & $\begin{array}{l}\alpha= \\
0.5\end{array}$ & 0.7 & 0.9 & $\begin{array}{c}\alpha \\
=1\end{array}$ & 1.3 & 0.5 & 0.7 & 0.9 & $\begin{array}{c}\alpha \\
=1\end{array}$ & 1.3 & 0.5 & 0.7 & 0.9 & $\begin{array}{c}\alpha \\
=1\end{array}$ & 1.3 & \\
\hline \multirow{2}{*}{$\begin{array}{l}\text { Sh } \\
\text { ift }\end{array}$} & $L=$ & 2.0 & 2.1 & 2.1 & 2.1 & 1.9 & 2.0 & 2.0 & 2.1 & 2.1 & 1.8 & 1.8 & 1.9 & 1.9 & 2.0 & 0.27 \\
\hline & $\begin{array}{c}1.93 \\
7\end{array}$ & 59 & 08 & 15 & & 11 & 33 & 95 & 1 & 11 & 07 & 46 & 38 & 72 & 36 & $\begin{array}{c}0711 \\
5\end{array}$ \\
\hline \multirow[t]{15}{*}{1} & 369. & 37 & 37 & 37 & 36 & 36 & 37 & 37 & 37 & 37 & 37 & 36 & 37 & 37 & 37 & 370. \\
\hline & 54 & 0.4 & 0.0 & 0.9 & 9.9 & 9.8 & 0.3 & 0.6 & 0.7 & 0.5 & 0.5 & 9.8 & 0.2 & 0.2 & 0.6 & 00 \\
\hline & & 1 & 3 & 5 & 4 & 7 & 0 & 3 & 4 & 3 & 9 & 2 & 4 & 4 & 6 & \\
\hline & 370. & 36 & 37 & 36 & 36 & 39 & 36 & 36 & 36 & 36 & 43 & 38 & 36 & 36 & 35 & 369. \\
\hline & 91 & 4.7 & 0.5 & 9.1 & 6.1 & 3.5 & 3.0 & 1.5 & 3.0 & 7.3 & 9.3 & 3.0 & 0.1 & 3.6 & 8.6 & 50 \\
\hline & & 2 & 9 & 3 & 8 & 8 & 5 & 4 & 8 & 7 & 0 & 2 & 2 & 2 & 7 & \\
\hline & 24 & 25 & 24 & 23 & 24 & 24 & 27 & 27 & 26 & 25 & 22 & 27 & 31 & 30 & 26 & 19 \\
\hline & 110 & 11 & 11 & 11 & 11 & 96 & 11 & 11 & 11 & 11 & 84 & 99 & 11 & 11 & 11 & 106 \\
\hline & & 4 & 4 & 4 & 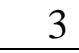 & & 4 & 5 & 5 & 5 & & & 5 & 5 & 6 & \\
\hline & 253 & 25 & 25 & 25 & 25 & 23 & 25 & 26 & 26 & 25 & 21 & 24 & 26 & 26 & 26 & 256 \\
\hline & & 9 & 6 & 7 & 6 & 9 & 9 & 1 & 0 & 9 & 4 & 6 & 2 & 1 & 4 & \\
\hline & 509 & 50 & 50 & 50 & 50 & 50 & 51 & 50 & 51 & 50 & 48 & 51 & 51 & 50 & 50 & 512 \\
\hline & & 9 & 2 & 7 & 5 & 7 & 0 & 8 & 0 & 5 & 8 & 2 & 0 & 6 & 7 & \\
\hline & 111 & 10 & 11 & 11 & 11 & 11 & 10 & 10 & 11 & 11 & 12 & 11 & 10 & 10 & 10 & 1107 \\
\hline & 0 & 92 & 10 & 12 & 16 & 62 & 93 & 93 & 09 & 06 & 73 & 28 & 74 & 86 & 92 & \\
\hline \multirow{14}{*}{$\begin{array}{c}\mathbf{0 .} \\
97 \\
5\end{array}$} & 230. & 24 & 26 & 26 & 28 & 20 & 22 & 24 & 25 & 27 & 18 & 20 & 22 & 23 & 25 & 344. \\
\hline & 85 & 9.8 & 5.6 & 9.5 & 9.3 & 6.2 & 5.8 & 3.9 & 3.0 & 0.8 & 7.6 & 4.1 & 3.7 & 1.2 & 6.1 & 70 \\
\hline & & 0 & 6 & $\mathbf{0}$ & 2 & 3 & 9 & 6 & 3 & 4 & 2 & 4 & 2 & 8 & 7 & \\
\hline & 220. & 24 & 26 & 26 & 28 & 19 & 21 & 23 & 24 & 26 & 18 & 19 & 21 & 21 & 24 & 344. \\
\hline & 86 & 1.2 & 1.8 & 5.9 & 7.2 & 9.3 & 3.0 & 3.2 & 3.9 & 5.8 & 9.3 & 6.3 & 1.0 & 8.2 & 7.4 & 20 \\
\hline & & 4 & 0 & 1 & $?$ & 0 & 7 & 8 & 7 & 9 & 5 & 8 & 4 & 6 & 1 & \\
\hline & 20 & 20 & 20 & 20 & 20 & 20 & 22 & 22 & 21 & 20 & 19 & 23 & 24 & 23 & 21 & 18 \\
\hline & 73 & 78 & 82 & 83 & 87 & 65 & 73 & 77 & 80 & 84 & 58 & 65 & 74 & 75 & 80 & 99 \\
\hline & 164 & 17 & 18 & 19 & 20 & 14 & 16 & 17 & 18 & 19 & 12 & 14 & 16 & 16 & 18 & 239 \\
\hline & & 8 & 8 & 0 & 2 & 6 & 2 & 4 & 0 & 1 & 9 & 4 & 0 & 7 & 1 & \\
\hline & 317 & 34 & 36 & 36 & 39 & 28 & 30 & 33 & 34 & 36 & 25 & 27 & 30 & 31 & 35 & 477 \\
\hline & & 0 & 2 & 7 & 6 & 2 & 7 & 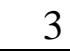 & 7 & 9 & 0 & 8 & 5 & 4 & 2 & \\
\hline & 663 & 74 & 78 & 79 & 85 & 59 & 64 & 71 & 74 & 79 & 56 & 59 & 62 & 65 & 75 & 1031 \\
\hline & & 0 & 0 & 1 & 5 & 7 & 5 & 0 & 3 & 4 & 0 & 0 & 9 & 7 & 0 & \\
\hline 0. & 153. & 17 & 19 & 20 & 22 & 13 & 14 & 16 & 17 & 20 & 11 & 12 & 14 & 15 & 17 & 320. \\
\hline 95 & 87 & 2.9 & 4.2 & 2.4 & 3.1 & 3.4 & 7.0 & 4.4 & 5.3 & 1.4 & 8.7 & 9.2 & 5.0 & 2.4 & 8.8 & 59 \\
\hline
\end{tabular}




\begin{tabular}{|c|c|c|c|c|c|c|c|c|c|c|c|c|c|c|c|c|}
\hline & & 3 & 6 & 7 & 8 & 6 & 4 & 6 & 8 & 8 & 2 & 4 & 4 & 6 & 2 & \\
\hline & 138. & 16 & 18 & 19 & 22 & 11 & 12 & 15 & 16 & 19 & 10 & 11 & 12 & 13 & 16 & 320. \\
\hline & 74 & 1.6 & 8.0 & 5.5 & 1.7 & 8.7 & 9.4 & 0.8 & 3.0 & 5.3 & 7.6 & 3.9 & 8.1 & 5.5 & 6.9 & 09 \\
\hline & & 3 & 5 & 9 & 8 & 1 & 4 & 9 & 7 & 9 & 6 & 2 & 9 & 9 & 7 & \\
\hline & 17 & 17 & 16 & 16 & 15 & 17 & 19 & 18 & 18 & 16 & 16 & 19 & 21 & 20 & 18 & 16 \\
\hline & 54 & 56 & 60 & 62 & 67 & 49 & 53 & 56 & 58 & 62 & 44 & 48 & 53 & 54 & 58 & 92 \\
\hline & 114 & 12 & 13 & 14 & 15 & 99 & 11 & 12 & 12 & 14 & 86 & 95 & 10 & 11 & 13 & 222 \\
\hline & & 7 & 9 & 6 & 7 & & 0 & 1 & 8 & 4 & & & 8 & 3 & 0 & \\
\hline & 209 & 23 & 26 & 27 & 30 & 17 & 19 & 22 & 24 & 27 & 15 & 17 & 19 & 20 & 24 & 444 \\
\hline & & 6 & 3 & 7 & 2 & 9 & 9 & 3 & 0 & 6 & 7 & 3 & 7 & 7 & 4 & \\
\hline & 429 & 49 & 56 & 58 & 67 & 37 & 40 & 46 & 50 & 58 & 33 & 35 & 40 & 42 & 51 & 959 \\
\hline & & 4 & 1 & 5 & 0 & 1 & 7 & 5 & 2 & 5 & 3 & 8 & 1 & 5 & 2 & \\
\hline 0. & 109. & 12 & 14 & 15 & 17 & 93. & 10 & 11 & 12 & 15 & 83. & 89. & 10 & 10 & 12 & 297. \\
\hline 92 & 92 & 4.8 & 2.8 & 1.6 & 5.5 & 98 & 3.3 & 7.2 & 6.0 & 0.1 & 07 & 05 & 0.9 & 7.9 & 8.3 & 63 \\
\hline 5 & & 7 & 8 & 2 & 2 & & 3 & 2 & 8 & 0 & & & 7 & 6 & 8 & \\
\hline & 94.9 & 11 & 13 & 14 & 17 & 77. & 88. & 10 & 11 & 14 & 68. & 71. & 85. & 94. & 11 & 297. \\
\hline & 8 & 3.1 & 5.2 & 4.6 & 1.8 & 26 & 10 & 5.7 & 7.2 & 4.7 & 20 & 94 & 22 & 13 & 9.3 & 13 \\
\hline & & 8 & 7 & 3 & 0 & & & 9 & 3 & 0 & & & & & 0 & \\
\hline & 15 & 15 & 14 & 14 & 13 & 15 & 16 & 16 & 15 & 14 & 14 & 17 & 18 & 18 & 15 & 15 \\
\hline & 42 & 44 & 46 & 48 & 53 & 38 & 41 & 42 & 43 & 47 & 34 & 38 & 41 & 41 & 44 & 85 \\
\hline & 83 & 91 & 10 & 10 & 12 & 72 & 77 & 84 & 90 & 10 & 63 & 68 & 75 & 79 & 92 & 206 \\
\hline & & & 2 & 8 & 4 & & & & & 7 & & & & & & \\
\hline & 148 & 17 & 19 & 20 & 24 & 12 & 13 & 15 & 17 & 20 & 11 & 11 & 13 & 14 & 17 & 412 \\
\hline & & 0 & 8 & 9 & 2 & 7 & 9 & 9 & 2 & 5 & 2 & 8 & 5 & 5 & 5 & \\
\hline & 300 & 35 & 40 & 44 & 50 & 24 & 27 & 32 & 35 & 43 & 21 & 23 & 27 & 29 & 36 & 890 \\
\hline & & 1 & 9 & 0 & 3 & 7 & 8 & 8 & 9 & 5 & 9 & 2 & 1 & 5 & 5 & \\
\hline 0. & 81.9 & 92. & 10 & 11 & 13 & 69. & 75. & 85. & 92. & 11 & 62. & 66. & 73. & 77. & 95. & 275. \\
\hline 9 & O & 42 & 7.5 & 5.8 & 7.6 & 70 & 38 & 98 & 78 & 3.6 & 45 & 26 & 74 & 92 & 28 & 79 \\
\hline & & & 9 & 6 & 4 & & & & & 7 & & & & & & \\
\hline & 66.5 & 81. & 99. & 10 & 13 & 53. & 59. & 74. & 82. & 10 & 47. & 49. & 57. & 64. & 86. & 275. \\
\hline & 8 & 94 & 55 & 9.1 & 1.6 & 59 & 95 & 81 & 89 & 7.0 & 41 & 37 & 96 & 33 & 64 & 29 \\
\hline & & & & 5 & 8 & & & & & 2 & & & & & & \\
\hline & 13 & 13 & 12 & 12 & 12 & 13 & 14 & 14 & 13 & 12 & 13 & 15 & 16 & 16 & 14 & 14 \\
\hline & 34 & 34 & 36 & 38 & 44 & 31 & 33 & 33 & 34 & 37 & 29 & 31 & 33 & 33 & 34 & 79 \\
\hline & 63 & 68 & 77 & 83 & 98 & 55 & 58 & 63 & 67 & 81 & 49 & 52 & 57 & 58 & 68 & 191 \\
\hline & 112 & 12 & 14 & 15 & 19 & 93 & 10 & 11 & 12 & 15 & 83 & 87 & 97 & 10 & 13 & 382 \\
\hline & & 5 & 8 & 9 & 0 & & 1 & 6 & 6 & 6 & & & & 4 & 0 & \\
\hline & 212 & 25 & 30 & 33 & 39 & 17 & 19 & 23 & 25 & 32 & 15 & 16 & 18 & 20 & 26 & 825 \\
\hline & & 3 & 4 & 0 & 3 & 5 & 5 & 4 & 9 & 8 & 6 & 3 & 9 & 5 & 3 & \\
\hline 0. & 49.3 & 54. & 63. & 69. & 87. & 43. & 45. & 50. & 54. & 67. & 39. & 41. & 44. & 46. & 55. & 235. \\
\hline 85 & & 44 & 84 & 17 & 00 & 51 & 88 & 02 & 00 & 31 & 69 & 70 & 84 & 38 & 44 & 39 \\
\hline & 36.0 & 43. & 56. & 62. & 82. & 29. & 31. & 38. & 44. & 60. & 25. & 26. & 30. & 32. & 46. & 234. \\
\hline & & 92 & 23 & 19 & 43 & 19 & 84 & 75 & 50 & 49 & 72 & 29 & 26 & 98 & 95 & 89 \\
\hline
\end{tabular}




\begin{tabular}{|c|c|c|c|c|c|c|c|c|c|c|c|c|c|c|c|c|}
\hline & 11 & 11 & 10 & 10 & 9 & 11 & 12 & 11 & 11 & 10 & 11 & 12 & 13 & 13 & 12 & 12 \\
\hline & 23 & 23 & 24 & 24 & 28 & 22 & 23 & 23 & 23 & 24 & 21 & 23 & 23 & 23 & 22 & 68 \\
\hline & 40 & 42 & 46 & 50 & 61 & 36 & 37 & 39 & 41 & 48 & 33 & 35 & 37 & 37 & 41 & 163 \\
\hline & 65 & 72 & 86 & 93 & $\begin{array}{r}11 \\
9\end{array}$ & 57 & 59 & 66 & 71 & 91 & 52 & 54 & 57 & 59 & 73 & 326 \\
\hline & 120 & 14 & 17 & 19 & 25 & 10 & 10 & 12 & 14 & 18 & 90 & 94 & 10 & 11 & 14 & 704 \\
\hline & & 2 & 6 & 4 & 2 & 0 & 9 & 5 & 3 & 9 & & & 4 & 3 & 9 & \\
\hline \multirow[t]{7}{*}{$\begin{array}{l}0 . \\
8\end{array}$} & $\begin{array}{r}33.4 \\
9\end{array}$ & $\begin{array}{r}35 . \\
41\end{array}$ & $\begin{array}{c}39 . \\
96\end{array}$ & $\begin{array}{r}43 . \\
47\end{array}$ & $\begin{array}{r}56 . \\
11\end{array}$ & $\begin{array}{r}30 . \\
48\end{array}$ & $\begin{array}{r}31 . \\
42\end{array}$ & $\begin{array}{c}32 . \\
80\end{array}$ & $\begin{array}{r}34 . \\
37\end{array}$ & $\begin{array}{r}42 . \\
12\end{array}$ & $\begin{array}{c}28 . \\
26\end{array}$ & $\begin{array}{r}29 . \\
54\end{array}$ & $\begin{array}{r}30 . \\
91\end{array}$ & $\begin{array}{c}31 . \\
37\end{array}$ & $\begin{array}{r}34 . \\
74\end{array}$ & $\begin{array}{r}199 . \\
15\end{array}$ \\
\hline & $\begin{array}{r}21.8 \\
5\end{array}$ & $\begin{array}{r}25 . \\
58\end{array}$ & $\begin{array}{r}32 . \\
80\end{array}$ & $\begin{array}{r}37 . \\
94\end{array}$ & $\begin{array}{r}51 . \\
64\end{array}$ & $\begin{array}{r}18 . \\
30\end{array}$ & $\begin{array}{r}18 . \\
99\end{array}$ & $\begin{array}{r}22 . \\
03\end{array}$ & $\begin{array}{r}25 . \\
04\end{array}$ & $\begin{array}{r}35 . \\
87\end{array}$ & $\begin{array}{r}16 . \\
48\end{array}$ & $\begin{array}{r}16 . \\
21\end{array}$ & $\begin{array}{c}17 . \\
50\end{array}$ & $\begin{array}{r}18 . \\
82\end{array}$ & $\begin{array}{r}25 . \\
84\end{array}$ & $\begin{array}{r}198 . \\
65\end{array}$ \\
\hline & 9 & 9 & 8 & 8 & 7 & 9 & 10 & 10 & 10 & 8 & 9 & 11 & 11 & 11 & 10 & 10 \\
\hline & 17 & 17 & 17 & 17 & 19 & 17 & 18 & 17 & 17 & 17 & 16 & 18 & 18 & 18 & 17 & 57 \\
\hline & 28 & 29 & 30 & 32 & 40 & 26 & 27 & 27 & 27 & 31 & 25 & 26 & 27 & 26 & 27 & 138 \\
\hline & 44 & 46 & 52 & 51 & 76 & 39 & 40 & 42 & 44 & 56 & 36 & 37 & 39 & 39 & 45 & 275 \\
\hline & 76 & 87 & $\begin{array}{r}10 \\
6\end{array}$ & $\begin{array}{r}11 \\
8\end{array}$ & $\begin{array}{r}15 \\
9\end{array}$ & 66 & 68 & 77 & 85 & $\begin{array}{r}11 \\
4\end{array}$ & 60 & 62 & 66 & 68 & 87 & 595 \\
\hline \multirow{9}{*}{$\begin{array}{l}\text { 0. } \\
7\end{array}$} & 18.7 & 18. & 19. & 20. & 24. & 17. & 18. & 17. & 17. & 19. & 16. & 17. & 18. & 18. & 17. & 138. \\
\hline & 6 & 60 & 39 & 36 & 90 & 78 & $\mathbf{0 3}$ & 86 & 98 & 68 & 82 & 72 & 49 & $\mathbf{0 3}$ & 98 & 24 \\
\hline & 10.0 & 10. & 12. & 14. & 20. & 8.6 & 8.4 & 8.9 & 9.7 & 13. & 7.9 & 7.5 & 7.5 & 7.7 & 9.8 & 137. \\
\hline & 1 & 55 & 60 & 33 & 44 & 9 & 6 & 9 & 8 & 48 & 7 & 3 & 7 & 5 & 0 & 74 \\
\hline & 7 & 7 & 6 & 6 & 5 & 7 & 8 & 8 & 7 & 7 & 7 & 8 & 9 & 9 & 8 & 7 \\
\hline & 11 & 11 & 10 & 10 & 10 & 11 & 12 & 11 & 11 & 10 & 11 & 12 & 13 & 12 & 11 & 40 \\
\hline & 17 & 16 & 16 & 16 & 19 & 16 & 16 & 16 & 15 & 16 & 15 & 16 & 16 & 16 & 15 & 95 \\
\hline & 24 & 23 & 25 & 26 & 33 & 22 & 22 & 22 & 22 & 25 & 21 & 22 & 22 & 22 & 22 & 191 \\
\hline & 38 & 39 & 45 & 48 & 66 & 35 & 34 & 36 & 37 & 47 & 32 & 32 & 33 & 33 & 38 & 413 \\
\hline \multirow{9}{*}{$\begin{array}{l}0 . \\
5\end{array}$} & 8.40 & 8.1 & 7.7 & 7.6 & 7.7 & 8.4 & 8.7 & 8.5 & 8.3 & 7.7 & 8.2 & 9.1 & 9.5 & 9.4 & 8.7 & 56.4 \\
\hline & & 4 & 8 & 7 & 2 & 4 & 6 & 5 & 4 & 8 & 5 & 1 & 7 & 6 & 3 & 2 \\
\hline & 3.15 & 2.9 & 2.9 & 3.0 & 3.8 & 2.8 & 2.6 & 2.4 & 2.4 & 2.6 & 2.7 & 2.4 & 2.2 & 2.1 & 2.1 & 55.9 \\
\hline & & 2 & 3 & 4 & 9 & 9 & 2 & 5 & 2 & 5 & 0 & 6 & 7 & 9 & 1 & 2 \\
\hline & 4 & 5 & 4 & 4 & 4 & 5 & 5 & 5 & 5 & 5 & 5 & 6 & 7 & 7 & 6 & 3 \\
\hline & 6 & 6 & 6 & 6 & 5 & 6 & 7 & 7 & 7 & 6 & 6 & 7 & 8 & 8 & 7 & 16 \\
\hline & 8 & 8 & 7 & 7 & 7 & 8 & 8 & 8 & 8 & 7 & 8 & 9 & 9 & 9 & 8 & 39 \\
\hline & 10 & 10 & 9 & 9 & 9 & 10 & 10 & 10 & 10 & 9 & 10 & 10 & 11 & 11 & 10 & 78 \\
\hline & 14 & 14 & 13 & 14 & 15 & 14 & 14 & 13 & 13 & 13 & 13 & 14 & 14 & 14 & 13 & 168 \\
\hline \multirow{8}{*}{$\begin{array}{c}0 . \\
25\end{array}$} & 4.19 & 4.2 & 4.1 & 4.0 & 3.7 & 4.4 & 4.8 & 5.0 & 4.9 & 4.6 & 4.4 & 5.2 & 5.8 & 5.9 & 5.7 & 10.4 \\
\hline & & 7 & 5 & 7 & 8 & 0 & 9 & 2 & 8 & 2 & 1 & 8 & 8 & 6 & 3 & 0 \\
\hline & 0.94 & 0.7 & 0.6 & 0.6 & 0.6 & 0.8 & 0.7 & 0.6 & 0.6 & 0.5 & 0.8 & 0.7 & 0.6 & 0.6 & 0.5 & 9.89 \\
\hline & & 9 & 7 & 4 & 4 & 9 & 6 & 6 & 2 & 9 & 5 & 2 & 6 & 2 & 7 & \\
\hline & 3 & 3 & 3 & 3 & 3 & 3 & 4 & 4 & 4 & 4 & 3 & 4 & 5 & 5 & 5 & 1 \\
\hline & 4 & 4 & 4 & 4 & 3 & 4 & 4 & 5 & 5 & 4 & 4 & 5 & 5 & 6 & 5 & 3 \\
\hline & 4 & 4 & 4 & 4 & 4 & 4 & 5 & 5 & 5 & 5 & 4 & 5 & 6 & 6 & 6 & 7 \\
\hline & 5 & 5 & 4 & 4 & 4 & 5 & 5 & 5 & 5 & 5 & 5 & 6 & 6 & 6 & 6 & 14 \\
\hline
\end{tabular}




\begin{tabular}{|l|l|l|l|l|l|l|l|l|l|l|l|l|l|l|l|l|}
\hline & 6 & 6 & 5 & 5 & 5 & 6 & 6 & 6 & 6 & 5 & 6 & 7 & 7 & 7 & 7 & 30 \\
\hline
\end{tabular}


into account the $S D R L$ relative to the $A R L$ when designing an EWMA chart; i.e.; they added the constraint that $S D R L \leq A R L$; this was done to avoid large variation in the runlength distribution. So, using their criterion for the optimal design of the EWMA chart for the proposed GWMA-TBE chart, the optimal design would consist of specifying the desired $A R L_{0}$ and $A R L_{1}$ values as well as the magnitude of the process shift $(\delta)$ that is anticipated and then select the $(q, \alpha, L)$ combination that provides the desired $A R L$ performance subject to the constraint that $S D R L \leq A R L$. We used both definitions to obtain the "near optimal design". Note that, we specifically refer to this as the "near optimal design" because in the way the design was carried out, i.e., the two parameters $q$ and $\alpha$ were varied over a certain range and for each $(q, \alpha)$ combination that was investigated the value of the charting constant, i.e., $L>0$, was obtained so that the attained in-control $A R L$ is close to the nominal or specified value $A R L_{0}^{*}$. This approach might exclude the "true" optimal design.

Using the results in Tables 3, 4 and 5, the "near optimal" design would be those $(q, \alpha, L)$ combinations that result in the smallest $A R L_{1}$ for a specified shift $(\delta)$ given the $A R L_{0}=370$ ; with or without the constraint $S D R L \leq A R L$.

Table 6 provides the "near optimal" combinations of the parameters $(q, \alpha, L)$ as well as the $A R L_{1}$ values for different $\delta$ and $k=1,2,3,4$ and 5. From Table 6, we observe the following:

a. For smaller shifts, i.e., for values of $\delta$ closer to 1, a larger value of $q$ (closer to 1) and a smaller value of $\alpha$ (closer to 0.5 ) works best. The converse also holds, i.e., for larger 
Table 6: Near optimal $(q, \alpha, L)$ combinations with corresponding $A R L$ values.

\begin{tabular}{|c|c|c|c|c|c|c|c|c|c|c|c|c|c|c|c|c|c|c|c|c|}
\hline \multirow{2}{*}{$\begin{array}{c}\text { No } \\
\text { constr } \\
\text { aint }\end{array}$} & \multicolumn{4}{|c|}{$k=1$} & \multicolumn{4}{|c|}{$k=2$} & \multicolumn{4}{|c|}{$k=3$} & \multicolumn{4}{|c|}{$\boldsymbol{k}=4$} & \multicolumn{4}{|c|}{$k=5$} \\
\hline & \multicolumn{2}{|c|}{$\operatorname{AR} L^{q}$} & $\alpha$ & $L$ & \multicolumn{2}{|c|}{$A R L q$} & $\alpha$ & $L$ & \multicolumn{2}{|c|}{$A R L q$} & $\alpha$ & $L$ & \multicolumn{2}{|c|}{$\operatorname{ARL} q$} & $\alpha$ & $L$ & \multicolumn{2}{|c|}{$\overline{A R L q}$} & \multicolumn{2}{|l|}{$\alpha$} \\
\hline 0.975 & \begin{tabular}{|c|}
23 \\
6.1 \\
3
\end{tabular} & $\begin{array}{l}0 . \\
9 \\
5\end{array}$ & \begin{tabular}{|l|}
0 \\
5 \\
\end{tabular} & $\begin{array}{c}1 . \\
55 \\
2\end{array}$ & $\begin{array}{c}20 \\
7.6 \\
0\end{array}$ & $\begin{array}{l}0 . \\
9 \\
5\end{array}$ & 5 & $\begin{array}{c}1 . \\
71 \\
7\end{array}$ & $\begin{array}{c}18 \\
7.6 \\
2\end{array}$ & $\begin{array}{l}0 . \\
9 \\
5\end{array}$ & 0 & $\begin{array}{c}1 . \\
80 \\
7\end{array}$ & $\begin{array}{c}17 \\
0.7 \\
8\end{array}$ & \begin{tabular}{|l|}
0. \\
9 \\
5 \\
\end{tabular} & 0 & $\begin{array}{c}1 . \\
85 \\
9\end{array}$ & $\begin{array}{c}16 \\
3.5 \\
9\end{array}$ & $\begin{array}{l}0 . \\
9 \\
5\end{array}$ & \begin{tabular}{|l|}
0 \\
5 \\
\end{tabular} & $\begin{array}{l}1 . \\
91\end{array}$ \\
\hline 0.95 & $\begin{array}{c}16 \\
7.9 \\
9\end{array}$ & $\begin{array}{l}0 . \\
9 \\
5\end{array}$ & 0 & $\begin{array}{c}1 . \\
55 \\
2\end{array}$ & $\begin{array}{c}13 \\
6.5 \\
1\end{array}$ & $\begin{array}{l}0 . \\
9 \\
5\end{array}$ & 0 & $\begin{array}{c}1 . \\
71\end{array}$ & $\begin{array}{c}11 \\
8.7 \\
2\end{array}$ & $\begin{array}{l}0 . \\
9 \\
5\end{array}$ & 0 & $\begin{array}{c}1 . \\
80 \\
7\end{array}$ & $\begin{array}{c}10 \\
3.0 \\
6\end{array}$ & \begin{tabular}{|c|}
0. \\
9 \\
5
\end{tabular} & 0 & $\begin{array}{c}1 . \\
85 \\
9\end{array}$ & $\begin{array}{c}95 . \\
71\end{array}$ & $\begin{array}{l}0 . \\
9 \\
5\end{array}$ & 0 & $\begin{array}{l}1 . \\
91\end{array}$ \\
\hline 0.925 & $\begin{array}{c}12 \\
6.8 \\
2\end{array}$ & $\begin{array}{l}0 . \\
9 \\
5\end{array}$ & \begin{tabular}{|l|}
0 \\
5 \\
\end{tabular} & $\begin{array}{c}1 . \\
55 \\
2\end{array}$ & $\begin{array}{l}98 . \\
68\end{array}$ & $\begin{array}{l}0 . \\
9 \\
5\end{array}$ & 0 & $\begin{array}{c}1 . \\
71 \\
7\end{array}$ & $\begin{array}{l}83 . \\
07\end{array}$ & $\begin{array}{l}0 . \\
9 \\
5\end{array}$ & 0 & $\begin{array}{c}1 . \\
80 \\
7\end{array}$ & $\begin{array}{c}71 . \\
36\end{array}$ & \begin{tabular}{|l|}
0. \\
9 \\
5
\end{tabular} & 0 & $\begin{array}{c}1 . \\
85 \\
9\end{array}$ & $\begin{array}{l}65 . \\
67\end{array}$ & $\begin{array}{l}0 . \\
9 \\
5\end{array}$ & \begin{tabular}{|l|}
0 \\
5 \\
5
\end{tabular} & $\begin{array}{l}1 . \\
91\end{array}$ \\
\hline 0.9 & $\begin{array}{c}99 . \\
91\end{array}$ & $\begin{array}{l}0 . \\
9 \\
5\end{array}$ & \begin{tabular}{|l|}
0 \\
5 \\
\end{tabular} & $\begin{array}{c}1 . \\
55 \\
2\end{array}$ & $\begin{array}{r}75 . \\
25\end{array}$ & $\begin{array}{l}0 . \\
9 \\
5\end{array}$ & 0 & $\begin{array}{l}1 . \\
71\end{array}$ & $\begin{array}{l}62 . \\
45\end{array}$ & $\begin{array}{l}0 . \\
9 \\
5\end{array}$ & 0 & $\begin{array}{c}1 . \\
80 \\
7\end{array}$ & $\begin{array}{c}53 \\
24\end{array}$ & \begin{tabular}{|c|}
0. \\
9 \\
5
\end{tabular} & 0 & $\begin{array}{c}1 . \\
85 \\
9\end{array}$ & $\begin{array}{c}48 . \\
59\end{array}$ & $\begin{array}{l}0 . \\
9 \\
5\end{array}$ & \begin{tabular}{|l|}
0 \\
5 \\
5
\end{tabular} & $\begin{array}{l}1 . \\
91\end{array}$ \\
\hline 0.85 & $\begin{array}{l}67 . \\
82\end{array}$ & $\begin{array}{l}0 . \\
9 \\
5\end{array}$ & 0 & $\begin{array}{c}1 . \\
55 \\
2\end{array}$ & $\begin{array}{l}49 . \\
24\end{array}$ & $\begin{array}{l}0 . \\
9 \\
5\end{array}$ & 0 & $\begin{array}{l}1 . \\
71\end{array}$ & $\begin{array}{c}39 . \\
69\end{array}$ & $\begin{array}{l}0 . \\
9 \\
5\end{array}$ & 0 & $\begin{array}{c}1 . \\
80 \\
7\end{array}$ & $\begin{array}{c}34 . \\
05\end{array}$ & $\begin{array}{l}0 . \\
9 \\
5\end{array}$ & 0 & $\begin{array}{c}1 . \\
85 \\
9\end{array}$ & $\begin{array}{l}30 . \\
36\end{array}$ & $\begin{array}{l}0 . \\
9 \\
5\end{array}$ & 0 & 91 \\
\hline 0.8 & $\begin{array}{c}50 . \\
15\end{array}$ & $\begin{array}{l}0 . \\
9 \\
5\end{array}$ & \begin{tabular}{|l|}
0 \\
5 \\
\end{tabular} & $\begin{array}{c}1 . \\
55 \\
2\end{array}$ & $\begin{array}{l}35 . \\
28\end{array}$ & $\begin{array}{l}0 . \\
9 \\
5\end{array}$ & 0 & 71 & $\begin{array}{l}28 . \\
26\end{array}$ & $\begin{array}{l}0 . \\
9 \\
5\end{array}$ & 0 & $\begin{array}{c}1 . \\
80 \\
7\end{array}$ & $\begin{array}{c}24 \\
02\end{array}$ & \begin{tabular}{|c|}
0. \\
9 \\
5 \\
\end{tabular} & 0 & $\begin{array}{c}1 . \\
85 \\
9\end{array}$ & $\begin{array}{l}21 . \\
31\end{array}$ & \begin{tabular}{|l|}
0. \\
9 \\
5 \\
\end{tabular} & \begin{tabular}{|l|}
0 \\
5 \\
\end{tabular} & $\begin{array}{l}1 . \\
91\end{array}$ \\
\hline 0.7 & $\begin{array}{c}31 . \\
51\end{array}$ & $\begin{array}{l}0 . \\
9 \\
5\end{array}$ & $\begin{array}{l}0 \\
5 \\
5\end{array}$ & $\begin{array}{c}1 . \\
55 \\
2\end{array}$ & $\begin{array}{l}21 . \\
42\end{array}$ & $\begin{array}{l}0 . \\
9 \\
5\end{array}$ & 0 & 71 & $\begin{array}{l}16 . \\
82\end{array}$ & $\begin{array}{l}0 . \\
9 \\
5\end{array}$ & 0 & $\begin{array}{c}1 . \\
80 \\
7\end{array}$ & $\begin{array}{l}14 . \\
21\end{array}$ & \begin{tabular}{|c|}
0. \\
9 \\
5
\end{tabular} & $\begin{array}{l}0 \\
\dot{5}\end{array}$ & $\begin{array}{c}1 . \\
85 \\
9\end{array}$ & $\begin{array}{l}12 . \\
46\end{array}$ & $\begin{array}{l}0 . \\
9 \\
5\end{array}$ & $\begin{array}{l}0 \\
5 \\
5\end{array}$ & $\begin{array}{l}1 . \\
91\end{array}$ \\
\hline 0.6 & $\begin{array}{l}21 . \\
96\end{array}$ & $\begin{array}{l}0 . \\
9 \\
5\end{array}$ & 0 & $\begin{array}{c}1 . \\
55 \\
2\end{array}$ & $\begin{array}{l}14 . \\
67\end{array}$ & $\begin{array}{l}0 . \\
9 \\
5\end{array}$ & 0 & $\begin{array}{l}1 . \\
71\end{array}$ & $\begin{array}{l}11 . \\
28\end{array}$ & $\begin{array}{l}0 . \\
9\end{array}$ & 1 & $\begin{array}{c}2 . \\
11 \\
1\end{array}$ & $\begin{array}{c}9.2 \\
2\end{array}$ & $\begin{array}{l}0 . \\
9\end{array}$ & 1 & $\begin{array}{c}2 . \\
15 \\
6\end{array}$ & $\begin{array}{c}7.8 \\
2\end{array}$ & \begin{tabular}{|c|}
0. \\
8
\end{tabular} & 1 & $\begin{array}{c}2 . \\
22 \\
2\end{array}$ \\
\hline 0.5 & $\begin{array}{l}16 . \\
23\end{array}$ & $\begin{array}{l}0 . \\
9 \\
5\end{array}$ & $\begin{array}{l}0 \\
5\end{array}$ & $\begin{array}{c}1 . \\
55 \\
2\end{array}$ & $\begin{array}{l}10 . \\
43\end{array}$ & $\begin{array}{l}0 . \\
9\end{array}$ & 1 & 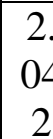 & $\begin{array}{c}7.6 \\
7\end{array}$ & $\begin{array}{l}0 . \\
8\end{array}$ & 1 & $\begin{array}{c}2 . \\
11 \\
5\end{array}$ & $\begin{array}{c}6.1 \\
8\end{array}$ & $\begin{array}{l}0 . \\
8\end{array}$ & 1 & $\begin{array}{c}2 . \\
16 \\
4\end{array}$ & $\begin{array}{c}5.2 \\
1\end{array}$ & \begin{tabular}{|c|}
0 \\
8
\end{tabular} & \begin{tabular}{l|}
1 \\
3 \\
\end{tabular} & $\begin{array}{c}2 . \\
21 \\
2\end{array}$ \\
\hline 0.33 & $\begin{array}{c}9.7 \\
4\end{array}$ & $\begin{array}{l}0 . \\
9\end{array}$ & $\begin{array}{l}1 \\
3 \\
\end{array}$ & $\begin{array}{c}1 . \\
88\end{array}$ & $\begin{array}{c}5.7 \\
9\end{array}$ & $\begin{array}{l}0 . \\
8\end{array}$ & 1 & 1. & $\begin{array}{c}4.2 \\
3\end{array}$ & $\begin{array}{l}0 . \\
7\end{array}$ & 1 & $\begin{array}{c}2 . \\
04 \\
9\end{array}$ & $\begin{array}{c}3.3 \\
7\end{array}$ & $\begin{array}{c}0 . \\
6\end{array}$ & 1 & $\begin{array}{c}2 . \\
07 \\
4\end{array}$ & $\begin{array}{c}2.7 \\
9\end{array}$ & \begin{tabular}{|c|} 
\\
5 \\
\end{tabular} & \begin{tabular}{|l|}
1 \\
3 \\
\end{tabular} & $\begin{array}{c}2 . \\
08 \\
8\end{array}$ \\
\hline 0.25 & $\begin{array}{c}7.4 \\
5\end{array}$ & $\begin{array}{l}0 . \\
8\end{array}$ & $\begin{array}{l}1 \\
3 \\
\end{array}$ & $\begin{array}{l}1 . \\
77\end{array}$ & $\begin{array}{c}4.4 \\
0\end{array}$ & $\begin{array}{l}0 . \\
7\end{array}$ & 1 & $\begin{array}{l}1 . \\
92\end{array}$ & $\begin{array}{c}3.1 \\
6\end{array}$ & $\begin{array}{c}0 . \\
5\end{array}$ & 1 & $\begin{array}{c}1 . \\
91 \\
4\end{array}$ & $\begin{array}{c}2.5 \\
6\end{array}$ & \begin{tabular}{|l}
0. \\
5
\end{tabular} & 1 & $\begin{array}{c}2 . \\
01 \\
6\end{array}$ & $\begin{array}{c}2.2 \\
4\end{array}$ & \begin{tabular}{|c|} 
\\
5
\end{tabular} & \begin{tabular}{|l|}
1 \\
3 \\
\end{tabular} & $\begin{array}{c}2 . \\
08 \\
8\end{array}$ \\
\hline SDRL & 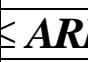 & $L k=$ & & & & $k=$ & & & & $k=$ & & & & $k=$ & & & & $k=$ & & \\
\hline & & & $\alpha$ & $L$ & $\overline{A R}$ & & $\alpha$ & $L$ & & & $\alpha$ & $L$ & $\boldsymbol{A R}$ & & $\alpha$ & $L$ & $\boldsymbol{A R}$ & & $\alpha$ & $L$ \\
\hline 0.975 & 27 & 0. & 0 & 1. & 23 & U & 0 & 1. & 21 & U. & 0 & 1. & 20 & 0. & 0 & 1. & 18 & 0. & 0 & 1. \\
\hline
\end{tabular}




\begin{tabular}{|c|c|c|c|c|c|c|c|c|c|c|c|c|c|c|c|c|c|c|c|c|}
\hline & $\begin{array}{c}0.1 \\
2\end{array}$ & 9 & 7 & $\begin{array}{c}80 \\
6\end{array}$ & $\begin{array}{c}7.0 \\
9\end{array}$ & $\begin{array}{l}9 \\
5\end{array}$ & 8 & $\begin{array}{c}85 \\
6\end{array}$ & $\begin{array}{c}4.9 \\
5\end{array}$ & $\begin{array}{l}9 \\
5\end{array}$ & 8 & $\begin{array}{c}89 \\
2\end{array}$ & $\begin{array}{c}5.0 \\
7\end{array}$ & $\begin{array}{l}9 \\
5\end{array}$ & $\dot{9}$ & $\begin{array}{c}95 \\
6\end{array}$ & $\begin{array}{c}5.1 \\
2\end{array}$ & $\begin{array}{l}9 \\
5\end{array}$ & 8 & $\begin{array}{c}94 \\
1\end{array}$ \\
\hline 0.95 & $\begin{array}{c}20 \\
2.6 \\
0\end{array}$ & $\begin{array}{l}0 . \\
9\end{array}$ & $\begin{array}{l}0 \\
7\end{array}$ & $\begin{array}{c}1 . \\
80 \\
6\end{array}$ & $\begin{array}{c}16 \\
0.6 \\
4\end{array}$ & $\begin{array}{l}0 . \\
9 \\
5\end{array}$ & 0 & $\begin{array}{c}1 . \\
85 \\
6\end{array}$ & $\begin{array}{c}13 \\
6.6 \\
6\end{array}$ & $\begin{array}{c}0 . \\
9 \\
5\end{array}$ & $\begin{array}{l}0 \\
8 \\
8\end{array}$ & $\begin{array}{c}1 . \\
89 \\
2\end{array}$ & $\begin{array}{c}12 \\
7.5 \\
5\end{array}$ & $\begin{array}{l}0 . \\
9 \\
5\end{array}$ & $\begin{array}{l}0 \\
\dot{9}\end{array}$ & $\begin{array}{c}1 . \\
95 \\
6\end{array}$ & $\begin{array}{c}10 \\
9.1 \\
6\end{array}$ & $\begin{array}{l}0 . \\
9 \\
5\end{array}$ & $\begin{array}{l}0 \\
8 \\
8\end{array}$ & $\begin{array}{c}1 . \\
94 \\
1\end{array}$ \\
\hline 0.925 & $\begin{array}{c}15 \\
7.9 \\
8\end{array}$ & $\begin{array}{l}0 . \\
9\end{array}$ & $\begin{array}{l}0 \\
7\end{array}$ & $\begin{array}{c}1 . \\
80 \\
6\end{array}$ & $\begin{array}{c}11 \\
5.4 \\
8\end{array}$ & $\begin{array}{l}0 . \\
9 \\
5\end{array}$ & $\begin{array}{l}0 \\
8 \\
8\end{array}$ & $\begin{array}{c}1 . \\
85 \\
6\end{array}$ & $\begin{array}{c}94 . \\
57\end{array}$ & $\begin{array}{c}0 . \\
9 \\
5\end{array}$ & $\begin{array}{l}0 \\
8 \\
8\end{array}$ & $\begin{array}{c}1 . \\
89 \\
2\end{array}$ & $\begin{array}{c}85 . \\
50\end{array}$ & $\begin{array}{l}0 . \\
9 \\
5\end{array}$ & $\begin{array}{l}0 \\
9\end{array}$ & $\begin{array}{c}1 . \\
95 \\
6\end{array}$ & $\begin{array}{l}72 . \\
86\end{array}$ & $\begin{array}{l}0 . \\
9 \\
5\end{array}$ & $\begin{array}{l}0 \\
8 \\
8\end{array}$ & $\begin{array}{c}1 . \\
94 \\
1\end{array}$ \\
\hline 0.9 & $\begin{array}{c}12 \\
5.3 \\
7\end{array}$ & $\begin{array}{l}0 . \\
9\end{array}$ & $\begin{array}{l}0 \\
7\end{array}$ & $\begin{array}{c}1 . \\
80 \\
6\end{array}$ & $\begin{array}{l}87 . \\
41\end{array}$ & $\begin{array}{l}0 . \\
9 \\
5\end{array}$ & $\begin{array}{l}0 \\
8 \\
8\end{array}$ & $\begin{array}{c}1 . \\
85 \\
6\end{array}$ & $\begin{array}{l}69 . \\
67\end{array}$ & $\begin{array}{l}0 . \\
9 \\
5\end{array}$ & $\begin{array}{l}0 \\
\dot{8}\end{array}$ & $\begin{array}{c}1 . \\
89 \\
2\end{array}$ & $\begin{array}{l}61 . \\
21\end{array}$ & $\begin{array}{l}0 . \\
9 \\
5\end{array}$ & $\begin{array}{l}0 \\
\text { 9 }\end{array}$ & $\begin{array}{c}1 . \\
95 \\
6\end{array}$ & $\begin{array}{c}52 . \\
56\end{array}$ & $\begin{array}{l}0 . \\
9 \\
5\end{array}$ & $\begin{array}{l}0 \\
8 \\
8\end{array}$ & $\begin{array}{c}1 . \\
94 \\
1\end{array}$ \\
\hline 0.85 & $\begin{array}{l}83 . \\
87\end{array}$ & $\begin{array}{l}0 . \\
9\end{array}$ & 7 & $\begin{array}{c}1 . \\
80 \\
6 \\
\end{array}$ & $\begin{array}{l}55 . \\
84\end{array}$ & $\begin{array}{l}0 . \\
9 \\
5\end{array}$ & $\begin{array}{l}0 \\
8 \\
\end{array}$ & $\begin{array}{c}1 . \\
85 \\
6\end{array}$ & $\begin{array}{l}43 . \\
27\end{array}$ & $\begin{array}{l}0 . \\
9 \\
5\end{array}$ & $\begin{array}{l}0 \\
8 \\
\end{array}$ & $\begin{array}{c}1 . \\
89 \\
2\end{array}$ & $\begin{array}{l}37 . \\
25\end{array}$ & $\begin{array}{l}0 . \\
9 \\
5\end{array}$ & $\begin{array}{l}0 \\
\text { 9 }\end{array}$ & $\begin{array}{c}1 . \\
95 \\
6\end{array}$ & $\begin{array}{l}32 . \\
24\end{array}$ & $\begin{array}{l}0 . \\
9 \\
5\end{array}$ & $\begin{array}{l}0 \\
8 \\
8\end{array}$ & $\begin{array}{c}1 . \\
94 \\
1\end{array}$ \\
\hline 0.8 & $\begin{array}{c}60 . \\
14\end{array}$ & $\begin{array}{l}0 . \\
9\end{array}$ & 7 & $\begin{array}{c}1 . \\
80 \\
6\end{array}$ & $\begin{array}{l}38 . \\
98\end{array}$ & $\begin{array}{l}0 . \\
9 \\
5\end{array}$ & $\begin{array}{l}0 \\
8 \\
\end{array}$ & $\begin{array}{c}1 . \\
85 \\
6\end{array}$ & $\begin{array}{l}30 . \\
30\end{array}$ & $\begin{array}{l}0 . \\
9 \\
5\end{array}$ & $\begin{array}{l}0 \\
8 \\
\end{array}$ & $\begin{array}{c}1 . \\
89 \\
2\end{array}$ & $\begin{array}{c}25 . \\
91\end{array}$ & $\begin{array}{l}0 . \\
9 \\
5\end{array}$ & $\begin{array}{l}0 \\
\dot{9}\end{array}$ & $\begin{array}{c}1 . \\
95 \\
6\end{array}$ & $\begin{array}{c}22 . \\
48\end{array}$ & $\begin{array}{c}0 . \\
9 \\
5\end{array}$ & $\begin{array}{l}0 \\
8 \\
\end{array}$ & $\begin{array}{c}1 . \\
94 \\
1\end{array}$ \\
\hline 0.7 & $\begin{array}{c}35 . \\
55\end{array}$ & $\begin{array}{l}0 . \\
9\end{array}$ & 0 & $\begin{array}{c}1 . \\
80 \\
6\end{array}$ & $\begin{array}{l}23 . \\
38\end{array}$ & $\begin{array}{l}0 . \\
9 \\
5\end{array}$ & $\begin{array}{l}0 \\
8 \\
\end{array}$ & $\begin{array}{c}1 . \\
85 \\
6 \\
\end{array}$ & $\begin{array}{l}17 . \\
86\end{array}$ & $\begin{array}{c}0 . \\
9\end{array}$ & $\begin{array}{l}0 \\
9 \\
\end{array}$ & $\begin{array}{c}2 . \\
09 \\
5\end{array}$ & $\begin{array}{c}14 . \\
70\end{array}$ & $\begin{array}{l}0 . \\
9\end{array}$ & 1 & $\begin{array}{c}2 . \\
14 \\
3\end{array}$ & $\begin{array}{l}12 . \\
64\end{array}$ & $\begin{array}{c}0 . \\
9\end{array}$ & 1 & $\begin{array}{c}2 . \\
17 \\
5\end{array}$ \\
\hline 0.6 & $\begin{array}{c}23 . \\
90\end{array}$ & $\begin{array}{l}0 . \\
9\end{array}$ & 0 & $\begin{array}{c}1 . \\
80 \\
6\end{array}$ & $\begin{array}{c}15 . \\
19\end{array}$ & $\begin{array}{c}0 . \\
9\end{array}$ & 1 & $\begin{array}{c}2 . \\
04 \\
5\end{array}$ & $\begin{array}{l}11 . \\
28\end{array}$ & $\begin{array}{c}0 . \\
9\end{array}$ & $\begin{array}{l}1 \\
3\end{array}$ & $\begin{array}{c}2 . \\
11 \\
1\end{array}$ & $\begin{array}{c}9.2 \\
2\end{array}$ & $\begin{array}{l}0 . \\
9\end{array}$ & $\begin{array}{l}1 \\
3\end{array}$ & $\begin{array}{c}2 . \\
15 \\
6\end{array}$ & $\begin{array}{c}7.8 \\
2\end{array}$ & $\begin{array}{c}0 . \\
8\end{array}$ & 1 & $\begin{array}{c}2 . \\
22 \\
2\end{array}$ \\
\hline 0.5 & $\begin{array}{l}16 . \\
84\end{array}$ & $\begin{array}{l}0 . \\
9\end{array}$ & 1 & $\begin{array}{c}1 . \\
90 \\
7\end{array}$ & $\begin{array}{l}10 . \\
43\end{array}$ & $\begin{array}{l}0 . \\
9\end{array}$ & $\begin{array}{l}1 \\
3\end{array}$ & $\begin{array}{c}2 . \\
04 \\
2\end{array}$ & $\begin{array}{c}7.6 \\
7\end{array}$ & $\begin{array}{c}0 . \\
8\end{array}$ & 1 & $\begin{array}{c}2 . \\
11 \\
5\end{array}$ & $\begin{array}{c}6.1 \\
8\end{array}$ & $\begin{array}{l}0 . \\
8\end{array}$ & $\begin{array}{l}1 \\
3\end{array}$ & $\begin{array}{c}2 . \\
16 \\
4\end{array}$ & $\begin{array}{c}5.2 \\
1\end{array}$ & $\begin{array}{c}0 . \\
8\end{array}$ & $\begin{array}{l}1 \\
3\end{array}$ & $\begin{array}{c}2 . \\
21 \\
2\end{array}$ \\
\hline 0.33 & $\begin{array}{c}9.7 \\
4\end{array}$ & $\begin{array}{l}0 . \\
9\end{array}$ & $\begin{array}{l}1 \\
3 \\
\end{array}$ & $\begin{array}{c}1 . \\
88\end{array}$ & $\begin{array}{c}5.7 \\
9\end{array}$ & $\begin{array}{l}0 . \\
8\end{array}$ & $\begin{array}{l}1 \\
3 \\
\end{array}$ & $\begin{array}{c}1 . \\
99 \\
9 \\
\end{array}$ & $\begin{array}{c}4.2 \\
3\end{array}$ & $\begin{array}{c}0 . \\
7\end{array}$ & $\begin{array}{l}1 \\
3 \\
\end{array}$ & $\begin{array}{c}2 . \\
04 \\
9 \\
\end{array}$ & $\begin{array}{c}3.4 \\
2\end{array}$ & $\begin{array}{l}0 . \\
5\end{array}$ & 1 & $\begin{array}{c}1 . \\
99 \\
9 \\
\end{array}$ & $\begin{array}{c}2.7 \\
9\end{array}$ & $\begin{array}{c}0 . \\
5\end{array}$ & $\begin{array}{l}1 \\
3 \\
\end{array}$ & $\begin{array}{c}2 . \\
08 \\
8 \\
\end{array}$ \\
\hline 0.25 & $\begin{array}{c}7.4 \\
5\end{array}$ & $\begin{array}{l}0 . \\
8\end{array}$ & 1 & $\begin{array}{c}1 . \\
77\end{array}$ & $\begin{array}{c}4.4 \\
0\end{array}$ & $\begin{array}{l}0 . \\
7\end{array}$ & 1 & $\begin{array}{c}1 . \\
92 \\
3\end{array}$ & $\begin{array}{c}3.1 \\
6\end{array}$ & $\begin{array}{c}0 . \\
5\end{array}$ & $\begin{array}{l}1 \\
3\end{array}$ & $\begin{array}{c}1 . \\
91 \\
4\end{array}$ & $\begin{array}{c}2.6 \\
7\end{array}$ & $\begin{array}{c}0 . \\
5\end{array}$ & 1 & $\begin{array}{c}1 . \\
99 \\
9\end{array}$ & $\begin{array}{c}2.2 \\
4\end{array}$ & $\begin{array}{c}0 . \\
5\end{array}$ & $\begin{array}{l}1 \\
3 \\
\end{array}$ & $\begin{array}{c}2 . \\
08 \\
8\end{array}$ \\
\hline
\end{tabular}


shifts, a smaller value of $q$ (closer to 0.5 ) and larger value of $\alpha$ (greater and equal to 1) works best;

b. The EWMA-TBE chart, i.e. when $\alpha=1$, only features as the optimal design for $\delta \leq 0.7$. This result confirms the fact that a GWMA chart can be designed to outperform an EWMA chart for very small, small and moderate shifts.

\subsection{The zero-state versus the steady-state average run-length}

The out-of-control average run-lengths $\left(A R L_{1}\right)$ of the previous sections are called the zero-state $A R L$ 's and are based on the assumption that the shift occurs at start-up, i.e., at time $t=1$. However, it is also of interest to see whether a GWMA-TBE chart designed for optimal performance at start-up works well if the shift occurs later in the process, say at time $t=50$, $100,150,300$, etc. This is called the steady-state performance and the $A R L$ is referred to as the steady-state $A R L$; the assumption is basically that a stable process has been operating in-control for some time before the shift occurs. We calculated the steady-state $A R L$ for some $(q, \alpha, L)$ combinations when $k=1,2$ and compared it to the zero-state $A R L$. The results are presented in Table 7 and from this table we observe the following:

i. The zero-state and steady-state $A R L$ are the same for all practical purposes. The minor differences that are observed are due to the inherent simulation variability;

ii. The GWMA-TBE chart generally performs similar or, in many cases, better than the EWMA-TBE chart when $\delta \geq 0.7$ (i.e., for very small, small or moderate shifts). For $\delta<0.7$ (i.e., for larger shifts), the EWMA-TBE chart generally performs better than the GWMATBE. These results hold irrespective of when the shift has occurred; 
Table 7: The zero-state and steady-state $A R L$ values for the GWMA-TBE chart.

\begin{tabular}{|c|c|c|c|c|c|c|c|c|c|c|c|c|c|c|c|}
\hline & & & & & \multicolumn{11}{|c|}{ Shift } \\
\hline $\boldsymbol{k}$ & $\begin{array}{c}\text { Time } \\
\text { of shift }\end{array}$ & $q$ & $\alpha$ & $L$ & $\begin{array}{l}0.9 \\
75\end{array}$ & $\begin{array}{c}0.9 \\
5\end{array}$ & $\begin{array}{l}0.9 \\
25\end{array}$ & 0.9 & $\begin{array}{c}0.8 \\
5\end{array}$ & 0.8 & 0.7 & 0.6 & 0.5 & $\begin{array}{c}0.3 \\
3\end{array}$ & $\begin{array}{c}0.2 \\
5\end{array}$ \\
\hline \multirow[t]{12}{*}{$k$} & $t=1$ & $\begin{array}{c}0.9 \\
0\end{array}$ & $\begin{array}{c}\text { 0.7 } \\
\text { 0 }\end{array}$ & $\begin{array}{l}1.8 \\
06\end{array}$ & $\begin{array}{c}270 \\
.1\end{array}$ & $\begin{array}{c}202 \\
.6\end{array}$ & $\begin{array}{c}158 \\
.0\end{array}$ & $\begin{array}{c}125 \\
.4\end{array}$ & $\begin{array}{c}83 . \\
9\end{array}$ & $\begin{array}{c}60 . \\
1\end{array}$ & $\begin{array}{c}35 . \\
6\end{array}$ & $\begin{array}{c}23 . \\
9\end{array}$ & $\begin{array}{c}17 . \\
3\end{array}$ & $\begin{array}{c}11 . \\
3\end{array}$ & 9.6 \\
\hline & & 0.9 & 1.0 & 1.9 & 295 & 238 & 192 & 156 & 105 & 74. & 40. & 25. & 16. & 10. & 8.9 \\
\hline & & 0 & 0 & 07 & .7 & .5 & .4 & .7 & .4 & 4 & 3 & 0 & 8 & 5 & \\
\hline & $t=\mathbf{5 0}$ & $\begin{array}{c}0.9 \\
0\end{array}$ & $\begin{array}{c}0.7 \\
0\end{array}$ & $\begin{array}{l}1.8 \\
06\end{array}$ & $\begin{array}{c}263 \\
.4\end{array}$ & $\begin{array}{c}199 \\
.2\end{array}$ & $\begin{array}{c}153 \\
.5\end{array}$ & $\begin{array}{c}123 \\
.4\end{array}$ & $\begin{array}{c}82 . \\
1\end{array}$ & $\begin{array}{c}59 . \\
3\end{array}$ & $\begin{array}{c}36 . \\
0\end{array}$ & $\begin{array}{c}24 . \\
3\end{array}$ & $\begin{array}{c}17 . \\
9\end{array}$ & $\begin{array}{c}11 . \\
9\end{array}$ & $\begin{array}{c}10 . \\
2\end{array}$ \\
\hline & & $\begin{array}{c}0.9 \\
0\end{array}$ & $\begin{array}{c}1.0 \\
0\end{array}$ & $\begin{array}{l}1.9 \\
07\end{array}$ & $\begin{array}{c}287 \\
.6\end{array}$ & $\begin{array}{c}231 \\
.3\end{array}$ & $\begin{array}{c}186 \\
.5\end{array}$ & $\begin{array}{c}152 \\
.4\end{array}$ & $\begin{array}{c}101 \\
.7\end{array}$ & $\begin{array}{c}72 . \\
0\end{array}$ & $\begin{array}{c}39 . \\
5\end{array}$ & $\begin{array}{c}24 . \\
3\end{array}$ & $\begin{array}{c}16 . \\
7\end{array}$ & $\begin{array}{c}10 . \\
5\end{array}$ & 9.0 \\
\hline & $\begin{array}{l}t= \\
100\end{array}$ & $\begin{array}{c}0.9 \\
0\end{array}$ & $\begin{array}{c}\text { 0.7 } \\
\text { 0 }\end{array}$ & $\begin{array}{l}1.8 \\
06\end{array}$ & $\begin{array}{c}266 \\
.4\end{array}$ & $\begin{array}{c}202 \\
.4\end{array}$ & $\begin{array}{c}157 \\
.9\end{array}$ & $\begin{array}{c}126 \\
.5\end{array}$ & $\begin{array}{c}85 . \\
1\end{array}$ & $\begin{array}{c}61 . \\
6\end{array}$ & $\begin{array}{c}36 . \\
6\end{array}$ & $\begin{array}{c}24 . \\
9\end{array}$ & $\begin{array}{c}18 . \\
4\end{array}$ & $\begin{array}{c}12 . \\
4\end{array}$ & $\begin{array}{c}10 . \\
6\end{array}$ \\
\hline & & $\begin{array}{c}0.9 \\
0\end{array}$ & $\begin{array}{c}1.0 \\
0\end{array}$ & $\begin{array}{l}1.9 \\
07\end{array}$ & $\begin{array}{c}290 \\
.2\end{array}$ & $\begin{array}{c}233 \\
.9\end{array}$ & $\begin{array}{c}188 \\
.3\end{array}$ & $\begin{array}{c}153 \\
.2\end{array}$ & $\begin{array}{c}103 \\
.4\end{array}$ & $\begin{array}{c}73 . \\
2\end{array}$ & $\begin{array}{c}39 . \\
5\end{array}$ & $\begin{array}{c}24 . \\
3\end{array}$ & $\begin{array}{c}16 . \\
8\end{array}$ & $\begin{array}{c}10 . \\
7\end{array}$ & 9.2 \\
\hline & $\begin{array}{l}t= \\
150\end{array}$ & $\begin{array}{c}0.9 \\
0\end{array}$ & $\begin{array}{c}0.7 \\
\text { 0 }\end{array}$ & $\begin{array}{l}1.8 \\
06\end{array}$ & $\begin{array}{c}266 \\
.4\end{array}$ & $\begin{array}{c}201 \\
.8\end{array}$ & $\begin{array}{c}157 \\
.0\end{array}$ & $\begin{array}{c}126 \\
.3\end{array}$ & $\begin{array}{c}86 . \\
0\end{array}$ & $\begin{array}{c}61 . \\
4\end{array}$ & $\begin{array}{c}37 . \\
1\end{array}$ & $\begin{array}{c}25 . \\
2\end{array}$ & $\begin{array}{c}18 . \\
7\end{array}$ & $\begin{array}{c}12 . \\
9\end{array}$ & $\begin{array}{c}11 . \\
4\end{array}$ \\
\hline & & $\begin{array}{c}0.9 \\
0\end{array}$ & $\begin{array}{c}1.0 \\
0\end{array}$ & $\begin{array}{l}1.9 \\
07\end{array}$ & $\begin{array}{c}289 \\
.0\end{array}$ & $\begin{array}{c}233 \\
.9\end{array}$ & $\begin{array}{c}189 \\
.6\end{array}$ & $\begin{array}{c}153 \\
.5\end{array}$ & $\begin{array}{c}103 \\
.0\end{array}$ & $\begin{array}{c}72 . \\
9\end{array}$ & $\begin{array}{c}39 . \\
4\end{array}$ & $\begin{array}{c}24 . \\
3\end{array}$ & $\begin{array}{c}16 . \\
9\end{array}$ & $\begin{array}{c}11 . \\
1\end{array}$ & 9.7 \\
\hline & $\begin{array}{l}t= \\
300\end{array}$ & $\begin{array}{c}0.9 \\
0\end{array}$ & $\begin{array}{c}0.7 \\
0\end{array}$ & $\begin{array}{l}1.8 \\
06\end{array}$ & $\begin{array}{c}272 \\
.7\end{array}$ & $\begin{array}{c}203 \\
.8\end{array}$ & $\begin{array}{c}159 \\
.2\end{array}$ & $\begin{array}{c}128 \\
.1\end{array}$ & $\begin{array}{c}86 . \\
9\end{array}$ & $\begin{array}{c}62 . \\
7\end{array}$ & $\begin{array}{c}37 . \\
9\end{array}$ & $\begin{array}{c}26 . \\
2\end{array}$ & $\begin{array}{c}19 . \\
4\end{array}$ & $\begin{array}{c}13 . \\
5\end{array}$ & $\begin{array}{c}11 . \\
9\end{array}$ \\
\hline & & $\begin{array}{c}0.9 \\
0\end{array}$ & $\begin{array}{c}1.0 \\
0\end{array}$ & $\begin{array}{l}1.9 \\
07\end{array}$ & $\begin{array}{c}287 \\
.1\end{array}$ & $\begin{array}{c}235 \\
.4\end{array}$ & $\begin{array}{c}191 \\
.0\end{array}$ & $\begin{array}{c}152 \\
.6\end{array}$ & $\begin{array}{c}103 \\
.0\end{array}$ & $\begin{array}{c}72 . \\
9\end{array}$ & $\begin{array}{c}39 . \\
7\end{array}$ & $\begin{array}{c}25 . \\
0\end{array}$ & $\begin{array}{c}17 . \\
4\end{array}$ & $\begin{array}{c}11 . \\
8\end{array}$ & $\begin{array}{c}10 . \\
7\end{array}$ \\
\hline & $\begin{array}{c}\text { Shewh } \\
\text { art }\end{array}$ & $\begin{array}{c}0.0 \\
0\end{array}$ & $\begin{array}{c}1.0 \\
0\end{array}$ & $\begin{array}{c}0.0 \\
03\end{array}$ & $\begin{array}{c}360 \\
8\end{array}$ & $\begin{array}{c}351 \\
5\end{array}$ & $\begin{array}{c}342 \\
3\end{array}$ & 333 & $\begin{array}{c}314 \\
6\end{array}$ & 296 & $\begin{array}{c}259 \\
2\end{array}$ & $\begin{array}{c}222 \\
2\end{array}$ & 185 & $\begin{array}{c}122 \\
4\end{array}$ & 92. \\
\hline \multirow[t]{7}{*}{$\boldsymbol{k}$} & $t=1$ & $\begin{array}{c}0.9 \\
0\end{array}$ & $\begin{array}{c}0.7 \\
0\end{array}$ & $\begin{array}{l}1.9 \\
60\end{array}$ & $\begin{array}{c}245 \\
.8\end{array}$ & $\begin{array}{c}170 \\
.7\end{array}$ & $\begin{array}{c}124 \\
.0\end{array}$ & $\begin{array}{c}94 . \\
0\end{array}$ & $\begin{array}{c}59 . \\
0\end{array}$ & $\begin{array}{c}40 . \\
5\end{array}$ & $\begin{array}{c}23 . \\
4\end{array}$ & $\begin{array}{c}15 . \\
6\end{array}$ & $\begin{array}{c}11 . \\
4\end{array}$ & 7.5 & 6.3 \\
\hline & & $\begin{array}{c}0.9 \\
0\end{array}$ & $\begin{array}{c}1.0 \\
0\end{array}$ & $\begin{array}{l}2.0 \\
45\end{array}$ & $\begin{array}{c}270 \\
.6\end{array}$ & $\begin{array}{c}201 \\
.2\end{array}$ & $\begin{array}{c}151 \\
.3\end{array}$ & $\begin{array}{c}115 \\
.8\end{array}$ & $\begin{array}{c}70 . \\
8\end{array}$ & $\begin{array}{c}46 . \\
6\end{array}$ & $\begin{array}{c}24 . \\
3\end{array}$ & $\begin{array}{c}15 . \\
2\end{array}$ & $\begin{array}{c}10 . \\
8\end{array}$ & 7.1 & 6.1 \\
\hline & $t=\mathbf{5 0}$ & $\begin{array}{c}0.9 \\
0\end{array}$ & $\begin{array}{c}0.7 \\
0\end{array}$ & $\begin{array}{l}1.9 \\
60\end{array}$ & $\begin{array}{c}240 \\
.0\end{array}$ & $\begin{array}{c}167 \\
.4\end{array}$ & $\begin{array}{c}122 \\
.4\end{array}$ & $\begin{array}{c}92 . \\
6\end{array}$ & $\begin{array}{c}58 . \\
1\end{array}$ & $\begin{array}{c}40 . \\
5\end{array}$ & $\begin{array}{c}23 . \\
8\end{array}$ & $\begin{array}{c}16 . \\
2\end{array}$ & $\begin{array}{c}12 . \\
0\end{array}$ & 8.2 & 7.3 \\
\hline & & $\begin{array}{c}0.9 \\
0\end{array}$ & $\begin{array}{c}1.0 \\
0\end{array}$ & $\begin{array}{r}2.0 \\
45 \\
\end{array}$ & $\begin{array}{c}263 \\
.8\end{array}$ & $\begin{array}{c}195 \\
.9\end{array}$ & $\begin{array}{c}146 \\
.0\end{array}$ & $\begin{array}{c}112 \\
.1\end{array}$ & $\begin{array}{c}68 . \\
6\end{array}$ & $\begin{array}{c}45 . \\
1\end{array}$ & $\begin{array}{c}23 . \\
6\end{array}$ & $\begin{array}{c}14 . \\
9\end{array}$ & $\begin{array}{c}10 . \\
7\end{array}$ & 7.3 & 6.6 \\
\hline & $\begin{array}{l}t= \\
100\end{array}$ & $\begin{array}{c}\mathbf{0 . 9} \\
\mathbf{0}\end{array}$ & $\begin{array}{c}0.7 \\
0\end{array}$ & $\begin{array}{l}1.9 \\
60\end{array}$ & $\begin{array}{c}242 \\
.4\end{array}$ & $\begin{array}{c}169 \\
.9\end{array}$ & $\begin{array}{c}124 \\
.6\end{array}$ & $\begin{array}{c}94 . \\
4\end{array}$ & $\begin{array}{c}59 . \\
8\end{array}$ & $\begin{array}{c}42 . \\
1\end{array}$ & $\begin{array}{c}24 . \\
7\end{array}$ & $\begin{array}{c}16 . \\
9\end{array}$ & $\begin{array}{c}12 . \\
7\end{array}$ & 9.1 & 8.3 \\
\hline & & $\begin{array}{c}0.9 \\
0\end{array}$ & $\begin{array}{c}1.0 \\
0\end{array}$ & $\begin{array}{l}2.0 \\
45\end{array}$ & $\begin{array}{c}261 \\
.3\end{array}$ & $\begin{array}{c}193 \\
.7\end{array}$ & $\begin{array}{c}145 \\
.1\end{array}$ & $\begin{array}{c}112 \\
.2\end{array}$ & $\begin{array}{c}68 . \\
9\end{array}$ & $\begin{array}{c}45 . \\
6\end{array}$ & $\begin{array}{c}24 . \\
0\end{array}$ & $\begin{array}{c}15 . \\
4\end{array}$ & $\begin{array}{c}11 . \\
2 .\end{array}$ & 8.0 & 7.3 \\
\hline & $\begin{array}{l}t= \\
150\end{array}$ & $\begin{array}{c}0.9 \\
0\end{array}$ & $\begin{array}{c}\mathbf{0 . 7} \\
\mathbf{0}\end{array}$ & $\begin{array}{l}1.9 \\
60\end{array}$ & $\begin{array}{c}242 \\
6\end{array}$ & 171 & $\begin{array}{c}124 \\
9\end{array}$ & $\begin{array}{c}95 . \\
0\end{array}$ & $\begin{array}{c}60 . \\
2\end{array}$ & $\begin{array}{c}42 . \\
2\end{array}$ & $\begin{array}{c}24 . \\
9\end{array}$ & $\begin{array}{c}17 . \\
2\end{array}$ & 13. & 9.6 & 8.8 \\
\hline
\end{tabular}




\begin{tabular}{|c|c|c|c|c|c|c|c|c|c|c|c|c|c|c|c|}
\hline & $\mathbf{0 . 9}$ & $\mathbf{1 . 0}$ & $\mathbf{2 . 0}$ & 260 & 194 & 145 & 111 & 68. & 45. & 24. & 15. & 11. & 8.4 & 8.0 \\
& $\mathbf{0}$ & $\mathbf{0}$ & $\mathbf{4 5}$ & .9 & .0 & .5 & .5 & 1 & 5 & 3 & 6 & 7 & & \\
\hline $\boldsymbol{t}=$ & $\mathbf{0 . 9}$ & $\mathbf{0 . 7}$ & $\mathbf{1 . 9}$ & 245 & 173 & 125 & 95. & 60. & 43. & 26. & 18. & 14. & 10. & 10. \\
$\mathbf{3 0 0}$ & $\mathbf{0}$ & $\mathbf{0}$ & $\mathbf{6 0}$ & .7 & .2 & .6 & 5 & 4 & 0 & 2 & 2 & 2 & 9 & 8 \\
\hline & $\mathbf{0 . 9}$ & $\mathbf{1 . 0}$ & $\mathbf{2 . 0}$ & 262 & 196 & 148 & 113 & 69. & 45. & 24. & 16. & 12. & 9.6 & 9.2 \\
& $\mathbf{0}$ & $\mathbf{0}$ & $\mathbf{4 5}$ & .5 & .3 & .9 & .3 & 7 & 9 & 9 & 3 & 4 & & \\
\hline Shewh & $\mathbf{0 . 0}$ & $\mathbf{1 . 0}$ & $\mathbf{0 . 0}$ & 352 & 334 & 317 & 301 & 269 & 239 & 185 & 137 & 97. & 44. & 26. \\
art & $\mathbf{0}$ & $\mathbf{0}$ & $\mathbf{7 5}$ & .2 & .8 & .9 & .4 & .7 & .8 & .2 & .7 & 2 & 6 & 8 \\
\hline
\end{tabular}


iii. Both the GWMA-TBE and EWMA-TBE charts outperform the Shewhart-TBE; this is a result that was also observed when studying the results of Tables 3, 4 and 5 with the zerostate $A R L$ 's.

Note that the charting statistics for the Shewhart-TBE chart are mutually independent, irrespective of the time the shift occurs, and therefore the time of shift can always be taken as $t=1$ when considering the OOC run-length.

\section{Phase II GWMA-TBE chart}

When the in-control (IC) value of the parameter $\theta$ is unknown, it is typically estimated from an in-control Phase I sample (the so-called reference or calibration sample) before prospective (i.e., online) monitoring starts in Phase II; this scenario is referred to as the "standard unknown" case and denoted by Case U. Note that the Phase I sample is taken when the process was thought to operate in-control and without any special causes of concern (see Montgomery (2009), page 198). The point estimate of $\theta$ is denoted by $\hat{\theta}$ and is used to estimate the starting value $Z_{0}$ and to estimate the Phase II lower control limit of the GWMA-TBE chart; in both cases, $\hat{\theta}$ is substituted for the known parameter value $\theta_{0}$. The charting statistic and estimated lower control limit of the GWMA-TBE chart in Case U are defined as follows:

$$
Z_{t}=\sum_{i=1}^{t}\left(q^{(i-1)^{\alpha}}-q^{i^{\alpha}}\right) X_{t-i+1}+q^{t^{\alpha}} k \hat{\theta} \text { for } t=1,2,3, \ldots
$$

and

$$
L \hat{C} L=k \hat{\theta}-L \sqrt{Q k \hat{\theta}^{2}}
$$


The Phase II GWMA-TBE chart operates in the same manner as the GWMA-TBE chart of Case $\mathrm{K}$, i.e., the charting statistic in (14) is plotted on a control chart with the $L \hat{C} L$ given in (15) and if a point plots on or below the lower control limit the chart signals and the process is declared OOC so that a search for assignable causes is started.

The following points should be noted:

i. Obtaining an in-control Phase I reference sample is an important problem in its own right, but we do not study this problem here. We assume that we have an in-control Phase I reference sample. The interested reader is referred to Chakraborti et al. (2008) for an in-depth overview of Phase I control charting procedures as well as the operation and implementation of these charts;

ii. To estimate the unknown value of $\theta$, a suitable point estimator is required. We use the Maximum Likelihood Estimator (MLE) which is also the Uniform Minimum Variance Unbiased Estimator (UMVUE). To this end, if we let $X_{1}, X_{2}, \ldots, X_{m} \sim \operatorname{iidGamma}(k, \theta)$ denote the IC Phase I reference sample of size $m \geq 1$, the MLE is given by $\hat{\theta}=\sum_{i=1}^{m} X_{i} / k m$ and follows a $\operatorname{Gamma}(\mathrm{km}, \theta / \mathrm{km})$ distribution;

iii. Because a point estimate is substituted for the unknown parameter value in Case $U$, the starting value and lower control limits are both random variables (as indicated by the ${ }^{\wedge}$ notation). Therefore, it is of interest to examine the effects of estimation on the Phase II runlength distribution and hence the performance and robustness of the GWMA-TBE chart using the design parameters of Case K. Stated differently, we want to know if the design parameters for the Case K GWMA-TBE chart may be used for the Phase II GWMA-TBE 
chart in Case U. To this end, it is important to stress that, given a point estimate $\hat{\theta}$ (calculated using an in-control Phase I sample), we obtain the conditional Phase II run-length distribution and we observe the conditional performance of the GWMA-TBE chart. That is, the observed performance of the chart is based on that specific in-control Phase I sample. Thus, the conditional performance and the conditional run-length distribution will be different for each practitioner based on his/her own in-control Phase I sample. Therefore, the conditional performance does not give us a complete insight into the overall performance of the chart. In order to get an overall picture and a more general idea about the effects of parameter estimation, we study the unconditional run-length distribution; this unconditional distribution can be thought of as the run-length distribution averaged over all possible values of the parameter estimates. Focusing on the average run-length $(A R L)$, we can write the aforementioned statements mathematically as follows:

$$
U A R L=E_{\hat{\theta}}(E(R \mid \hat{\theta}))=\int_{0}^{\infty} E(R \mid \hat{\theta}) f(\hat{\theta}) d \hat{\theta}
$$

where $U A R L$ denotes the unconditional $A R L, E(R \mid \hat{\theta})$ denotes the conditional $A R L$, i.e., the expectation of the run-length $(R)$ conditional on a point estimate $\hat{\theta}$, and $f(\hat{\theta})$ denotes the p.d.f. of $\hat{\theta}$. Similar expressions can be obtained for other characteristics of the run-length distribution as well.

\subsection{Effects of parameter estimation on the performance of the Phase II GWMA-TBE chart}

To assess if the design parameters for the Case K GWMA-TBE chart may be used for the Phase II GWMA-TBE chart in Case U, a simulation study was performed to calculate the in-control 
and out-of-control average run-length of the Phase II GWMA-TBE chart. Without loss of generality, we simulated the in-control Phase I sample from a $\operatorname{Gamma}(k, 1)$ distribution. We used $m=50,100,500$ and 1000 with four sets of design parameters:

i. $(k=1, q=0.95, \alpha=0.5, L=1.552),(k=1, q=0.95, \alpha=1, L=1.859)$,

ii. $(k=2, q=0.95, \alpha=0.5, L=1.717),(k=2, q=0.95, \alpha=1, L=1.944)$.

Note that the first and second sets use $k=1$ whereas the third and fourth sets use $k=2$. Also, the first and third sets result in GWMA-TBE charts whereas the second and fourth sets result in EWMA-TBE charts.

The results are displayed in panels (a), (b), (c) and (d) of Figure 1. These graphs display the $A R L$ curves, i.e., $A R L$ on the vertical axis versus the size of the shift $(\delta)$ on the horizontal axis. The graphs also display the corresponding $A R L$-curve of Case $\mathrm{K}$ and is used as the reference to which we compare the $A R L$-curves of Case U. The $A R L$ values are shown in the data tables below the graphs for ease of reference. From these graphs, we observe the following points:

i. The in-control $A R L$ in Case $\mathrm{U}$ is substantially larger than the corresponding in-control $A R L$ in Case $\mathrm{K}$; in some cases even greater than 1,000. The out-of-control $A R L$ in Case $\mathrm{U}$ is also larger than the corresponding out-of-control $A R L$ of Case K. Only if $\delta \leq 0.5$ do we see that the charts perform relatively similar;

ii. The smaller the in-control Phase I reference sample is, i.e., the smaller the value of $m$, the larger the difference between the Case $\mathrm{U}$ and Case $\mathrm{K}$ and $A R L$ is. For the Case $\mathrm{U}$ chart (based on the design parameters of the Case $\mathrm{K}$ chart), to perform anything like the Case $\mathrm{K}$ chart requires more than 1,000 Phase I observations; 

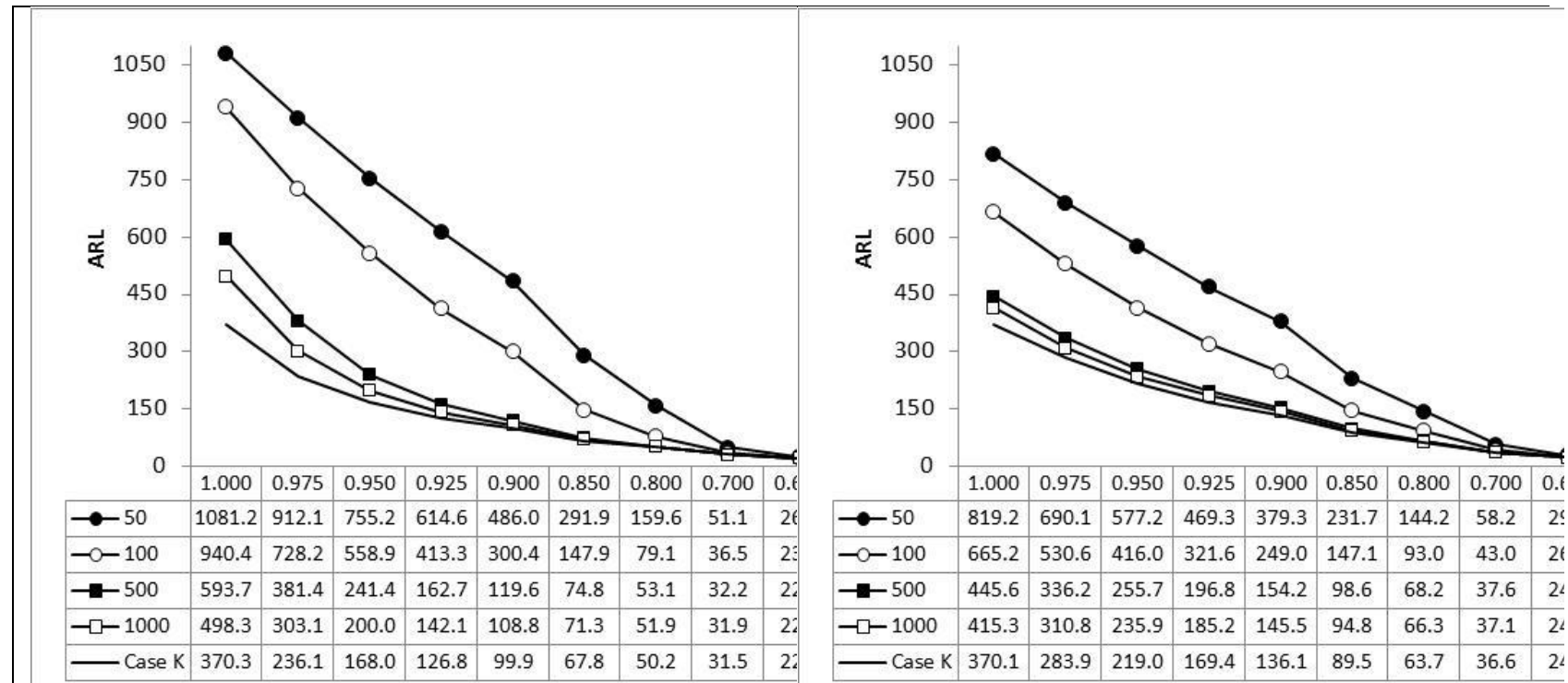

(a) $(k=1, q=0.95, \alpha=0.5, L=1.552)$

(b) $(k=1, q=0.95, \alpha=0.5, L=1.859)$

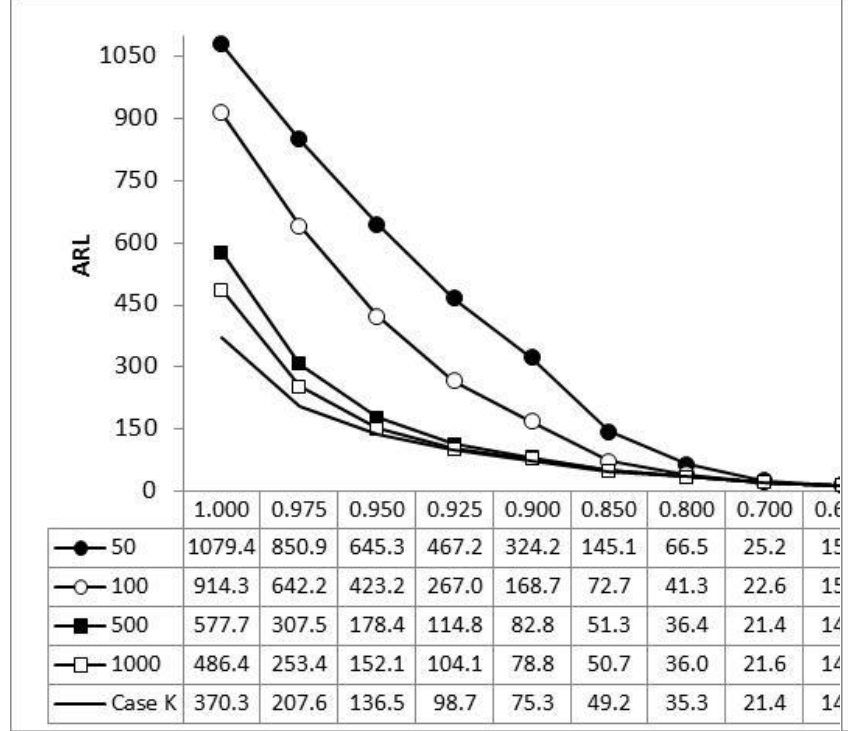

(c) $(k=2, q=0.95, \alpha=0.5, L=1.717)$

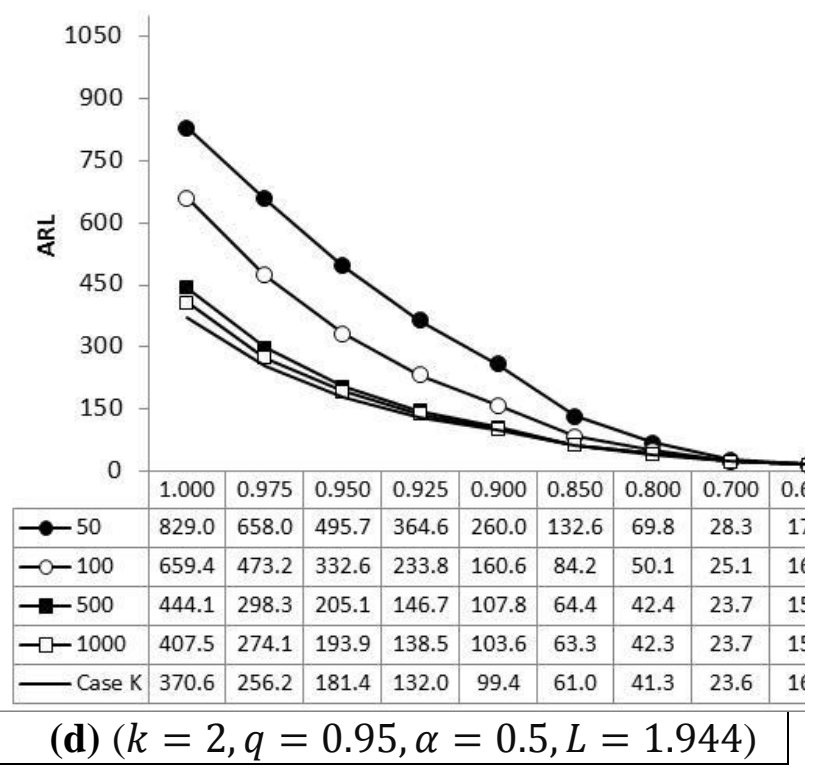

Figure 1: $A R L$ values for the GWMA-TBE chart for unknown $\theta$ when $k=1$ and 2 . 
iii. The EWMA-TBE chart is less impacted (compared to the GWMA-TBE chart) by the estimation from Phase I which is seen by looking at the $A R L$ values on the vertical axis.

The above observations demonstrate that in those scenarios where it is unrealistic to wait a long time to gather the necessary Phase I observations, an alternative and more realistic approach is needed since we cannot use the Case $\mathrm{K}$ design parameters. To this end, one should adjust the control limit, i.e., contract the control limit. This is investigated next.

\subsection{The in-control (IC) design of the Phase II GWMA-TBE chart}

The design of the Phase II GWMA-TBE chart requires one to use expression (16) and adjust the width of the lower control limit $(L)$ so that the in-control unconditional $A R L$ is equal to a prespecified value such as 370. This means we want to solve for the value of $L$ that satisfies the following equation:

$$
U A R L_{0}=E_{\hat{\theta}}(E(R \mid \hat{\theta}, I C))=\int_{0}^{\infty} E(R \mid \hat{\theta}, I C) f(\hat{\theta}) d \hat{\theta}=370 .
$$

The integral in equation (17) can be approximated by using computer simulation and calculating the average of a sufficiently large number of in-control conditional average run-lengths, i.e.,

calculating $\frac{1}{N} \sum_{j=1}^{N} E(R \mid \hat{\theta}, I C), N$ denotes the number of simulations and $E(R \mid \hat{\theta}, I C)$ denotes the conditional in-control average run-length. Using a search algorithm, we have found the value of $L$ that satisfies $\frac{1}{N} \sum_{j=1}^{N} E(R \mid \hat{\theta}, I C) \approx 370$ for some $(q, \alpha)$ combinations. These values are displayed in Table 8 along with the value of $L$ for the Case $\mathrm{K}$ chart; the latter correspond to the values listed in Table 2. From Table 8, we observe that the value of $L$ converges to the Case $\mathrm{K}$ 
Table 8: Design parameters for the Phase II GWMA-TBE chart in Case U.

\begin{tabular}{|c|c|c|c|c|c|c|c|}
\hline & & & \multicolumn{5}{|c|}{$m$} \\
\hline$k$ & $q$ & $\alpha$ & 50 & 100 & 500 & 1000 & $\begin{array}{c}\text { Case } \\
\mathbf{K}\end{array}$ \\
\hline \multirow[t]{2}{*}{1} & \multirow{4}{*}{0.95} & 0.50 & 0.927 & 1.099 & 1.387 & 1.463 & 1.552 \\
\hline & & 1.00 & 1.383 & 1.586 & 1.789 & 1.819 & 1.859 \\
\hline \multirow[t]{2}{*}{2} & & 0.50 & 1.020 & 1.240 & 1.551 & 1.625 & 1.717 \\
\hline & & 1.00 & 1.422 & 1.649 & 1.868 & 1.901 & 1.944 \\
\hline
\end{tabular}


value as the number of observations increases. So, the smaller the value of $m$, the narrower the control limits should be to compensate for the large $A R L$ we observed in Figure 1.

In the next two sections, we discuss a generalization of the proposed GWMA chart and an illustrative example is provided in order to demonstrate the application of the proposed chart.

\section{GWMA-TBE for monitoring the variance}

The proposed GWMA chart based on the $\operatorname{Gamma}(k, \theta)$ distribution can also be used to monitor the known in-control (IC) variance of a normal distribution for a sustained downward step shift. To this end, if $X_{i j}$ denotes the $j^{\text {th }}$ observation in the $i^{\text {th }}$ sample of size $n \geq 1$, where $X_{i j} \sim \operatorname{iidN}\left(\mu, \sigma_{0}^{2}\right)$ for $i=1,2,3, \ldots, j=1,2, \ldots, n$, and $\sigma_{0}^{2}$ is the known in-control (IC) standard/value for the variance, the statistic $\frac{(n-1) S_{i}^{2}}{2 \sigma_{0}^{2}}$ is distributed as $\operatorname{Gamma}\left(k=\frac{n-1}{2}, \theta_{0}=1\right)$, where $S_{i}^{2}=\frac{1}{n-1} \sum_{j=1}^{n}\left(X_{i j}-\bar{X}_{i}\right)^{2}$ denotes the sample variance. If the mean of the normal distribution is known beforehand and equal to $\mu_{0}$, the sample variance will be calculated as $S_{i}^{2}=\frac{1}{n} \sum_{j=1}^{n}\left(X_{i j}-\mu_{0}\right)^{2} \quad$ and the statistic $\frac{n S_{i}^{2}}{2 \sigma_{0}^{2}} \quad$ is distributed Gamma $\left(k=\frac{n}{2}, \theta_{0}=1\right)$. So, the design parameters given in Table 2 can be used to construct a GWMA chart to monitor the known variance. For example, if samples of size $n=5$ is taken from a normal distribution with known variance and unknown mean, it follows that $\frac{2 S_{i}^{2}}{\sigma_{0}^{2}} \sim \operatorname{Gamma}(2,1)$. So, using Table 2 and setting $(q=0.95, \alpha=1.0, L=1.944)$ will result in an 
EWMA chart with an in-control $A R L$ of 370.60 . The corresponding out-of-control $A R L$ performance of the EWMA chart, for monitoring the variance, can be found from Table 4 with $\delta=\sigma_{1}^{2} / \sigma_{0}^{2}$. Note that, in this case, when $\delta<1$, there would have been a decrease in the variance which would be equivalent to an improvement in the process.

Note that if the variance is unknown and has to be estimated from an in-control Phase I reference sample using the pooled variance estimator $S_{p}^{2}$ with $v_{1}$ degrees of freedom, for example the statistic $\frac{v_{1} S_{i}^{2}}{v_{2} S_{p}^{2}}$ follows an $F_{v_{2}, v_{1}}$ distribution, where $v_{2}$ denotes the degrees of freedom for a future or Phase II sample variance $S_{i}^{2}$. The proposed GWMA-TBE chart is, however, not suited for this scenario.

\section{Illustrative example}

To illustrate the application of the proposed GWMA-TBE chart, a simulated dataset is used. Because the proposed GWMA-TBE chart is for monitoring a high-performance process, for which failure rate is assumed to be very small, the known value of the in-control (IC) process failure rate is taken as $1 / \theta_{0}=1 / 5000=0.0002$; this implies the time until the $k^{\text {th }}$ failure, $X$, follows a $\operatorname{Gamma}(k, 5000)$ distribution.

The simulated dataset consists of 50 random observations from a $\operatorname{Gamma}(k=2,4000)$ distribution. If we assume that the known value of $\theta$ is 5000 , the simulated dataset may be viewed as observations from an out-of-control (OOC) process following a shift of $\delta=$ $4000 / 5000=0.8$; this is a deterioration in the process. Note that, because $k=2$, we will be 
monitoring the total time between two consecutive failures. Also, because the preceding developments assumed that $\theta=1$, the simulated data has to be scaled by dividing by $\theta_{0}=5000$.

Two sets of design parameters are used: $(q=0.9, \alpha=0.7, L=1.960)$ and $(q=0.9, \alpha=1.0$, $L=2.045$ ). The first set results in a GWMA-TBE chart whereas the second set results in an EWMA-TBE chart (because $\alpha=1.0$ ) with smoothing parameter $\lambda=1-0.9=0.1$. These two sets of parameters are chosen for the illustration purposes only of the proposed chart and any other combination can be chosen in this regard. However, while choosing a parameter combination in practice, it is important to note that, a larger value of $q$ and a smaller value of $\alpha$ usually works well for smaller shifts, i.e., for values of $\delta$ closer to 1 .

From Table 2, we can see that both charts are designed so that their $A R L_{0} \approx 370$. From Table 4, we observe that the GWMA-TBE chart has an OOC $A R L$ of 40.53 while the EWMA-TBE chart has an OOC $A R L$ of 46.63. So, we would expect the GWMA-TBE chart to signal before the EWMA-TBE chart.

The lower control limits are calculated using equation (9) and are equal to 1.546 and 1.336 , respectively. The charting statistics are calculated using equation (4) with $\theta_{0}=1$; this means the starting value is taken as 2 .

Figure 2 displays the GWMA-TBE and EWMA-TBE charts. From this figure, we observe that the GWMA-TBE chart signals at time 30 whereas the EWMA-TBE chart signals at time 34. Note that it is expected that the GWMA-TBE chart outperforms the EWAM chart for these choices of the design parameters. 


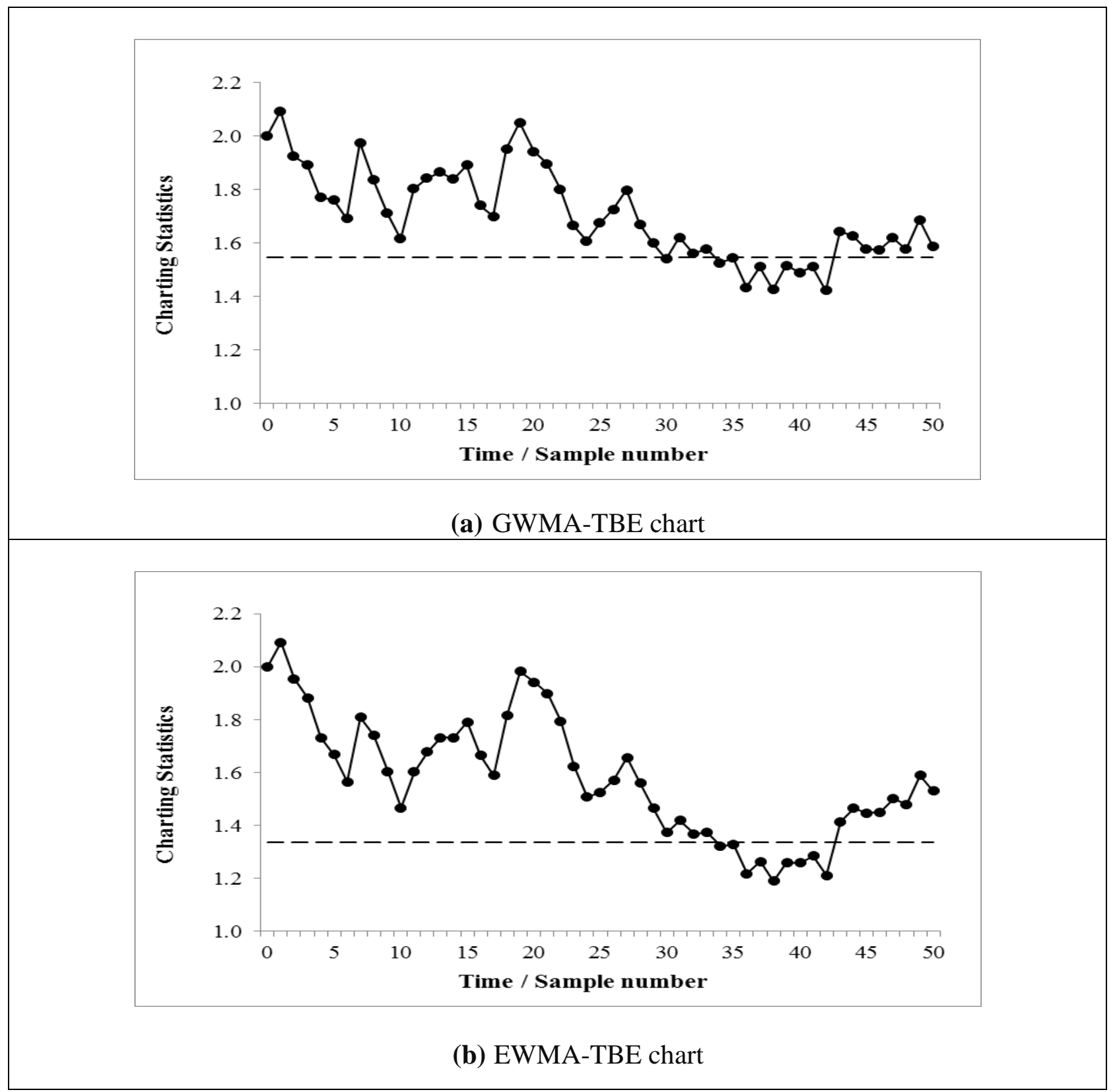

Figure 2: GWMA-TBE chart and EWMA-TBE chart for the example. 


\section{Concluding remarks}

Efficient control charts are crucial to improve the quality of a process. To this end, an efficient control chart should detect any change as quickly as possible since the faster a change is detected the quicker a corrective action can be taken. However, when monitoring nonconformities or defects, the traditional Shewhart-type attribute charts (such as the $c$-chart and the $p$-chart) are known to be inefficient at detecting small changes quickly and poor to detect changes when the failure rate is very small. For this reason, we have proposed a time-weighted chart, which sequentially accumulates all the information over time, and monitor the time between events (TBE), i.e., the time between consecutive defects. Accumulating all historical information provides greater sensitivity to detect small changes effectively. We have specifically proposed a one-sided Generally Weighted Moving Average (GWMA) control chart based on the gamma distribution to monitor the TBE; this chart has been called the GWMA-TBE chart. It has been shown that the proposed GWMA chart includes the one-sided Exponentially Weighted Moving Average (EWMA) and Shewhart-type charts as special cases. We have investigated the scenarios when the scale parameter of the gamma distribution is known (referred to as the "standard known") as well as when it is unknown (referred to as the "standard unknown"). It has been shown that when one estimates the unknown parameter from an in-control Phase I reference sample, the run-length (and in particular the $A R L$ ) is adversely affected. Three methods for calculating the run-length distribution and the associated characteristics of the run-length distribution have been investigated; this includes (i) Exact closed-form expressions, (ii) A Markov chain approach, and (iii) Monte Carlo Simulation. Due to the difficulties of numerically evaluating the closed-form expression and using the Markov chain approach for the GWMA- 
TBE chart, we have used computer simulation. To aid the implementation of the chart, the necessary design parameters for Case $\mathrm{K}$ and Case $\mathrm{U}$ have been provided, which guarantees that the in-control ARL is equal to a specified nominal value. An extensive performance analysis has been carried-out and a set of near optimal design parameters have been provided. The performance analysis has shown that the GWMA-TBE chart is better than the well-known EWMA and Shewhart charts at detecting very small to moderate changes. We have also shown how the GWMA-TBE chart can be used to monitor the variance of a normal distribution.

\section{Acknowledgement:}

This research was supported in part by STATOMET (Grant number: SMB2015A), University of Pretoria, South Africa. The authors gratefully acknowledge and appreciate the effort by two anonymous referees for providing valuable comments and sugestions that helped improve this work. 


\section{References}

Aslam, M., Al-marshadi, A. H., Jun, C. H. (2015). Monitoring process mean using generally weighted moving average chart for exponentially distributed characteristics. Communications in Statistics-Simulation and Computation DOI: 10.1080/03610918.2015.1102936.

Balakrishnan, N., Paroissin, C., Turlot, J. C. (2015). One-sided control charts based on precedence and weighted precedence statistics. Quality and Reliability Engineering International 31(1): 113-134.

Chakraborti S, Human S. W., Graham M. A. (2009). Phase I statistical process control charts: An overview and some results. Quality Engineering 21: 52-62.

Chakraborty, N., Chakraborti, S., Human, S. W., Balakrishnan, N. (2016). A generally weighted moving average signed-rank control chart. Quality and Reliability Engineering International DOI: $10.1002 /$ qre. 1968.

Chan, L. K., Zhang, J. (2000). Some issues in the design of EWMA charts. Communications in Statistics-Simulation and Computation 29(1): 201-217.

Chiu, W. C. (2009). Generally weighted moving average control charts with fast initial response features. Journal of Applied Statistics 36(3): 255-275.

Chiu, W. C., Sheu, S.H. (2008). Fast initial response features for Poisson GWMA control charts. Communications in Statistics-Simulation and Computation 37(7): 1422-1439.

Fu, J.C., Lou, W.W. (2003). Distribution Theory of Runs and Patterns and Its Applications: A Finite Markov Chain Imbedding Approach. World Scientific publishing, Singapore. 
Gan, F. F. (1998). Designs of one-and two-sided exponential EWMA charts. Journal of Quality Technology 30(1): 55.

Gan, F. F. (1994). Design of optimal exponential CUSUM control charts. Journal of Quality Technology 26(2): 109-124.

Gan, F. F., Choi, K. P. (1994). Computing average run lengths for exponential CUSUM schemes. Journal of Quality Technology 26(2): 134-143.

Graham, M.A., Chakraborti, S., Human, S.W. (2011a). A nonparametric EWMA sign chart for location based on individual measurements. Quality Engineering 23(3): 227-241.

Graham, M.A., Chakraborti, S., Human, S.W. (2011b). A nonparametric exponentially weighted moving average signed-rank chart for monitoring location. Computational Statistics \& Data Analysis 55(8): 2490-2503.

Hsu, B. M., Lai, P. J., Shu, M. H., Hung, Y. Y. (2009). A comparative study of the monitoring performance for weighted control charts. Journal of Statistics and Management Systems 12(2): 207-228.

Huang, C. J. (2014). A sum of squares generally weighted moving average control chart. Communications in Statistics-Theory and Methods 43(23): 5052-5071.

Huang, C. J., Tai, S. H., Lu, S. L. (2014). Measuring the performance improvement of a double generally weighted moving average control chart. Expert Systems with Applications 41(7): 33133322.

Human, S.W., Graham, M.A. (2007). Average run-lengths and operating characteristic curves. In Encyclopedia of Statistics in Quality and Reliability, Vol. 1, pp. 159-168, John Wiley \& Sons, Hoboken, New Jersey. 
Lu, S. L. (2015). An extended nonparametric exponentially weighted moving average sign control chart. Quality and Reliability Engineering International 31(1): 3-13.

Lucas, J. M., Saccucci, M. S. (1990). Exponentially weighted moving average control schemes: properties and enhancements. Technometrics 32(1): 1-12.

Maravelakis, P. E., Castagliola, P. (2009). An EWMA chart for monitoring the process standard deviation when parameters are estimated. Computational Statistics \& Data Analysis 53(7): 26532664.

Montgomery, D. C. (2009). Statistical Quality Control: A Modern Introduction. 7th ed. John Wiley \& Sons, Hoboken, New Jersey.

Nakagawa, T., Osaki, S. (1975). The discrete Weibull distribution. IEEE Transactions on Reliability 5: 300-301.

Sheu, S. H., Chiu, W. C. (2007). Poisson GWMA control chart. Communications in StatisticsSimulation and Computation 36(5): 1099-1114.

Sheu, S. H., Hsieh, Y. T. (2009). The extended GWMA control chart. Journal of Applied Statistics 36(2): 135-147.

Sheu, S. H., Yang, L. (2006). The generally weighted moving average control chart for monitoring the process median. Quality Engineering 18(3): 333-344.

Sheu, S. H., Huang, C. J., Hsu, T. S. (2012). Extended maximum generally weighted moving average control chart for monitoring process mean and variability. Computers \& Industrial Engineering 62(1): 216-225. 
Sheu, S. H., Huang, C. J., Hsu, T. S. (2013). Maximum chi-square generally weighted moving average control chart for monitoring process mean and variability. Communications in StatisticsTheory and Methods 42(23): 4323-4341.

Sheu, S. H., Lin, T. C. (2003). The generally weighted moving average control chart for detecting small shifts in the process mean. Quality Engineering 16: 209-231.

Teh, S. Y., Khoo, M. B., Wu, Z. (2012). Monitoring process mean and variance with a single generally weighted moving average chart. Communications in Statistics-Theory and Methods 41(12): 2221-2241.

Vardeman, S., Ray, D. O. (1985). Average run lengths for CUSUM schemes when observations are exponentially distributed. Technometrics 27(2): 145-150.

Xie, M., Goh, T. N., Ranjan, P. (2002). Some effective control chart procedures for reliability monitoring. Reliability Engineering \& System Safety 77(2): 143-150.

Zhang, C. W., Xie, M., Goh, T. N. (2005). Economic design of exponential charts for time between events monitoring. International Journal of Production Research 43(23): 5019-5032. Zhang, C. W., Xie, M., Liu, J. Y., Goh, T. N. (2007). A control chart for the Gamma distribution as a model of time between events. International Journal of Production Research 45(23): 56495666. 


\section{Appendix}

\section{A1. Decreasing weights}

We consider two scenarios:

i. For $\alpha=1$ and $0<q<1$, we have $w_{i}=q^{(i-1)}-q^{i}=q^{i-1}(1-q)$ which is decreasing function of $\mathrm{i}$ because $0<q<1$;

ii. For $0<\alpha<1$ and $0<q<1$, we have $q^{(i-1)^{\alpha}}>q^{i^{\alpha}}$ for all $i$. We can then re-write the weights $w_{i}=q^{(i-1)^{\alpha}}-q^{i^{\alpha}}=q^{(i-1)^{\alpha}}\left(1-q^{i^{\alpha}-(i-1)^{\alpha}}\right)$. Now, because $q^{(i-1)^{\alpha}}$ is a decreasing function for all $i, w_{i}$ will be decreasing if the remaining part of the product, i.e., $\left(1-q^{i^{\alpha}-(i-1)^{\alpha}}\right)$, is decreasing. To show this, we need to show that the exponent of $q$ viz., $w_{i}^{*}=\left(i^{\alpha}-(i-1)^{\alpha}\right)$ is decreasing for $i=1,2,3, \ldots$ and $0<\alpha<1$.

It can be easily shown that for $n \geq 1$ non-decreasing pairs of positive real numbers $\left(a_{i}, b_{i}\right), i=$ 1(1) $n$, such that $a_{i} \leq b_{i} \forall i=1(1) n$,

$$
\frac{\left(\prod_{i=1}^{n} a_{i}\right)+\left(\prod_{i=1}^{n} b_{i}\right)}{2} \geq \prod_{i=1}^{n} \frac{a_{i}+b_{i}}{2}
$$

If $a_{i}=a$ and $b_{i}=b$ for all $i$ such that $a \leq b$, then we can re-write the inequality in (i) as

$$
\frac{a^{n}+b^{n}}{2} \geq\left(\frac{a+b}{2}\right)^{n}
$$

Taking $b=(i+1)^{\frac{1}{n}}, a=(i-1)^{\frac{1}{n}}$ in (ii) for some integer $i \geq 1$, we get 


$$
i \geq\left(\frac{(i+1)^{\frac{1}{n}}+(i-1)^{\frac{1}{n}}}{2}\right)^{n} .
$$

Since $\alpha<1$, we can write $\alpha=\frac{1}{n}$ for some positive integer $n>1$ and replacing $\alpha=\frac{1}{n}$ in (iii), we $\quad$ get, $\quad i^{\alpha} \geq \frac{(i+1)^{\alpha}+(i-1)^{\alpha}}{2} \Rightarrow i^{\alpha}-(i-1)^{\alpha} \geq(i+1)^{\alpha}-i^{\alpha} \Rightarrow w_{i}^{*} \geq w_{i+1}^{*} . \quad$ Therefore, $w_{i}^{*}=i^{\alpha}-(i-1)^{\alpha}$ is decreasing for $i=1,2,3, \ldots$ and $0<\alpha<1$. This implies that $1-q^{i^{\alpha}-(i-1)^{\alpha}}$ is decreasing since $0<q<1$. Thus, we have $w_{i}=q^{(i-1)^{\alpha}}\left(1-q^{i^{\alpha}-(i-1)^{\alpha}}\right)$ to be decreasing for $i=1,2,3, \ldots$ and $0<\alpha<1$, which completes the proof.

A2. $\boldsymbol{Q}_{t}=\sum_{i=1}^{\infty}\left(\boldsymbol{q}^{(i-1)^{\alpha}}-\boldsymbol{q}^{i^{\alpha}}\right)^{2}$ is convergent as $\boldsymbol{t} \rightarrow \infty$, for $0 \leq \boldsymbol{q}<1, \boldsymbol{\alpha}>0$ We have $Q_{t}=\sum_{i=1}^{t}\left(q^{(i-1)^{\alpha}}-q^{i^{\alpha}}\right)^{2}=\sum_{i=1}^{t} q^{2(i-1)^{\alpha}}+\sum_{i=1}^{t} q^{2 i^{\alpha}}-2 \sum_{i=1}^{t} q^{(i-1)^{\alpha}} q^{i^{\alpha}}$. We consider two scenarios:

i. For $q=0, \lim _{t \rightarrow \infty} Q_{t}=\sum_{i=1}^{\infty}\left(q^{(i-1)^{\alpha}}-q^{i^{\alpha}}\right)^{2}=1$ which is trivial.

ii. For $0<q<1$, we have $q^{(i-1)^{\alpha}}>q^{i^{\alpha}} \Rightarrow q^{(i-1)^{\alpha}} q^{i^{\alpha}}>q^{2 i^{\alpha}}$. Substituting $q^{(i-1)^{\alpha}} q^{i^{\alpha}}$ by $q^{2 i^{\alpha}}$, it follows that $Q_{t}<\left(\sum_{i=1}^{t} q^{2(i-1)^{\alpha}}+\sum_{i=1}^{t} q^{2 i^{\alpha}}-2 \sum_{i=1}^{t} q^{2 i^{\alpha}}\right)=\sum_{i=1}^{t} q^{2(i-1)^{\alpha}}-\sum_{i=1}^{t} q^{2 i^{\alpha}}$. It then follows that $Q_{t}<\left(1-q^{2 t^{\alpha}}\right)<1$ 
So, for $0<q<1$, the monotonically increasing sequence $\left\{Q_{t} ; t=1,2,3, \ldots\right\}$ is bounded above and is therefore convergent. Further, $0<Q_{t}<1$ for all values of $t$, which implies that $0<\lim _{t \rightarrow \infty} Q_{t}<1$ and so there exists a number (denoted by $Q$ ) in the interval $(0,1)$ such that $\lim _{t \rightarrow \infty} Q_{t}=Q$ for $0<q<1$. This completes the proof.

A3. $\operatorname{Pr}[R=r]=I_{r-1}-I_{r}$, where $I_{r}=\operatorname{Pr}\left[\cap_{i=1}^{r} A_{i}^{c}\right]$ for $r=2,3,4, \ldots$

$\operatorname{Pr}[R=r]=\operatorname{Pr}\left[\left\{\bigcap_{i=1}^{r-1} A_{i}^{c}\right\} \cap A_{r}\right]=\operatorname{Pr}\left[\bigcap_{=1}^{r-1} A_{i}^{c}\right]-\operatorname{Pr}\left[\left\{\bigcap_{i=1}^{r-1} A_{i}^{c}\right\} \cap A_{i}^{c}\right]=\operatorname{Pr}\left[\cap_{i=1}^{r-1} A_{i}^{c}\right]-\operatorname{Pr}\left[\cap_{i=1}^{r} A_{i}^{c}\right]$

. Therefore, $\operatorname{Pr}[R=r]=I_{r-1}-I_{r}$, where $I_{r}=\operatorname{Pr}\left[\cap_{i=1}^{r} A_{i}^{c}\right]$.

A4. $A R L=1+\sum_{r=1}^{\infty} \mathbf{I}_{\mathrm{r}}$

Because $\operatorname{Pr}[R=r]=I_{\mathrm{r}-1}-I_{\mathrm{r}}$, we have $A R L=\sum_{r=1}^{\infty} r \operatorname{Pr}[R=r]$. Upon expanding and re-arranging some of the terms, we obtain

$A R L=\operatorname{Pr}[R=1]+\sum_{r=2}^{\infty} r \operatorname{Pr}[R=r]=1-\operatorname{Pr}\left[A_{1}^{c}\right]+\sum_{r=2}^{\infty} r\left(I_{\mathrm{r}-1}-I_{\mathrm{r}}\right)=1-\operatorname{Pr}\left[A_{1}^{c}\right]+I_{1}+\sum_{r=1}^{\infty} I_{\mathrm{r}}=1+\sum_{r=1}^{\infty} I_{\mathrm{r}}$, as required. 